\title{
UNCOUNTABLY MANY VARIETIES OF COMPLETELY SIMPLE SEMIGROUPS WITH METABELIAN SUBGROUPS
}

\author{
JIŘÍ KAĎOUREK* \\ Department of Mathematics, Masaryk University, Janáčkovo nám. 2a, 60200 Brno, Czech Republic \\ e-mail:kadourek@math.muni.cz
}

(Received 28 October, 2005; revised 24 March, 2006; accepted 2 May, 2006)

\begin{abstract}
It has been proved by D. E. Cohen [1] that the lattice of all varieties of metabelian groups is countable. In this paper, we show that the lattice of all varieties of completely simple semigroups with metabelian subgroups has the cardinality of the continuum. M. Petrich and N.R. Reilly have introduced in [6] the notion of near varieties of idempotent generated completely simple semigroups. The mapping assigning to every variety $\mathcal{V}$ of completely simple semigroups the class of all idempotent generated members of $\mathcal{V}$ is a complete lattice homomorphism of the lattice of all varieties of completely simple semigroups onto the lattice of all near varieties of idempotent generated completely simple semigroups. In this paper we show that, in fact, the lattice of all near varieties of idempotent generated completely simple semigroups with metabelian subgroups has itself the cardinality of the continuum.
\end{abstract}

2000 Mathematics Subject Classification. Primary 20M07. Secondary 20E10.

Introduction. Completely simple semigroups form a subclass of the wider class of all completely regular semigroups. Completely regular semigroups are semigroups which are unions of (mutually disjoint) groups. Any completely regular semigroup $S$ can thus be viewed as a semigroup with an additional unary operation assigning to every element of $S$ its inverse in the maximal subgroup of $S$ where this element occurs. Considerd in this way, all completely regular semigroups form a variety of unary semigroups, when they are treated as algebras with the operations of multiplication and inversion. Notice that the necessity to deal with completely regular semigroups as with unary semigroups in this context is enforced by the demand that varieties should be closed under the formation of subalgebras. As far as homomorphic images are concerned, it does not matter if completely regular semigroups are viewed as ordinary semigroups or as unary semigroups. Every homomorphism of completely regular semigroups preserves by itself the unary operation of inversion.

Recall that a non-empty subset $I$ of a semigroup $S$ is an ideal of $S$ if it has the property that $S I \subseteq I$ and $I S \subseteq I$. A semigroup $S$ is said to be simple if it has no proper ideal. Now completely simple semigroups are precisely semigroups which are at the same time completely regular and simple. Viewed as unary semigroups in the way described above, all completely simple semigroups form a proper subvariety of the variety of all completely regular semigroups. Completely simple semigroups can

*Research partially supported by the Ministry of Education of the Czech Republic under the project MSM 0021622409. 
also be characterized as rectangular bands of groups. For more information on this subject, we refer the reader to the monograph [8] by M. Petrich and N. R. Reilly.

Yet a substantially larger class of semigroups than the one consisting of all completely regular semigroups is the well-established class of all regular semigroups. Notice, in this context, that if completely regular semigroups are treated as unary semigroups, then unary subsemigroups of completely regular semigroups are the same as regular subsemigroups of completely regular semigroups. Thus we will often use the latter terminology to express this circumstance. If $S$ is a regular semigroup, then its subsemigroup generated by all its idempotents is well known to be a regular semigroup again - see the paper [2] by D. G. Fitz-Gerald. Therefore the subsemigroup of any completely regular semigroup $S$ generated by all idempotents of $S$ is a completely regular semigroup again, even if, this time, the given completely regular semigroup $S$ is considered only as an ordinary semigroup and only multiplication is used to generate the mentioned subsemigroup from the idempotents of $S$.

An ordinary semigroup $S$ is idempotent generated if it is generated by a set of its idempotents (merely by means of multiplication). In particular, this viewpoint applies to regular semigroups. As far as completely regular semigroups are concerned, if they are treated as unary semigroups, it would seem appropriate to say that a completely regular semigroup $S$ is idempotent generated if it is generated by a set of its idempotents using both operations of multiplication and inversion. However, from the previous remarks it becomes evident that one can dispense with the application of the operation of inversion in this particular context. Namely, it turns out that a completely regular semigroup $S$ is idempotent generated as a unary semigroup if and only if it is idempotent generated as an ordinary semigroup. To put it a bit more precisely, a completely regular semigroup $S$ is idempotent generated by means of multiplication and inversion if and only if $S$ is generated from the set of all its idempotents using only multiplication. Thus, in the case of completely regular semigroups, it does not matter if they are viewed as ordinary semigroups or as unary semigroups, when idempotent generated semigroups are considered.

A class $\mathcal{W}$ of idempotent generated completely regular semigroups is said to be a near variety of idempotent generated completely regular semigroups, if $\mathcal{W}$ is closed under the formation of idempotent generated regular subdirect products of arbitrary collections of semigroups, under the formation of idempotent generated regular subsemigroups, and under the formation of arbitrary homomorphic images. It can be easily verified that the direct product of any finite collection of idempotent generated completely regular semigroups is an idempotent generated completely regular semigroup again. Thus any near variety $\mathcal{W}$ of idempotent generated completely regular semigroups is closed under the formation of direct products of finite collections of semigroups. The class of all idempotent generated completely regular semigroups and the class of all idempotent generated completely simple semigroups are examples of near varieties of idempotent generated completely regular semigroups. However, as it will be shown later in this paper, neither of these two classes is closed under the formation of direct products of infinite collections of semigroups. Such direct products need not remain idempotent generated. Therefore the original definition of near varieties from [6], which imposed the requirement that near varieties should be closed under the formation of direct products of arbitrary families of semigroups, must be amended in the way specified above in this paragraph. And this must be done so even if the definition of near varieties is restricted, as in [6], only to the class of all idempotent generated completely simple semigroups. 
It will be shown in the next section of this paper that the mapping assigning to every variety $\mathcal{V}$ of completely regular semigroups the class $\mathcal{I} \mathcal{V}$ of all idempotent generated members of $\mathcal{V}$ is a complete lattice homomorphism of the lattice of all varieties of completely regular semigroups onto the lattice of all near varieties of idempotent generated completely regular semigroups. Restricted to the lattice of all varieties of completely simple semigroups, this result has already been obtained in [6]. Later in this paper, a continuum of near varieties of idempotent generated completely simple semigroups with metabelian subgroups will be provided. Hence, naturally, also a continuum of varieties of completely simple semigroups with metabelian subgroups will emerge.

Even more striking results in this direction will be obtained. For any distinct prime numbers $p, q$, let $\mathcal{A}_{p} \circ \mathcal{A}_{q}$ be the Malcev product of the varieties $\mathcal{A}_{p}$ and $\mathcal{A}_{q}$ of all abelian groups of exponent $p$ and $q$, respectively. Of course, $\mathcal{A}_{p} \circ \mathcal{A}_{q}$ is a variety of metabelian groups. It has been shown by G. Higman in [3] already that this variety $\mathcal{A}_{p} \circ \mathcal{A}_{q}$ is generated by a finite group. Consequently, according to the famous results of $\mathrm{S}$. Oates and M. B. Powell [5], $\mathcal{A}_{p} \circ \mathcal{A}_{q}$ is a Cross variety, which incidentally means that this variety has only finitely many subvarieties. We refer the reader also to the last chapter in the monograph [4] by $\mathrm{H}$. Neumann for more details on this subject. In contrast to these facts, in the last section of this paper, a continuum of near varieties of idempotent generated completely simple semigroups with subgroups in the group variety $\mathcal{A}_{p} \circ \mathcal{A}_{q}$ just treated will actually be obtained. As before, this clearly yields also a continuum of varieties of completely simple semigroups with subgroups in the mentioned variety $\mathcal{A}_{p} \circ \mathcal{A}_{q}$.

It is worth noticing that, for any distinct prime numbers $p, q$, the variety $\mathcal{A}_{p} \circ \mathcal{A}_{q}$ consists of groups whose exponents divide $p q$. Consequently, in any completely simple semigroup $S$ with subgroups in $\mathcal{A}_{p} \circ \mathcal{A}_{q}$, the unary operation of inversion coincides with the unary operation assigning to every element $a \in S$ its power $a^{p q-1}$. Therefore, the result on varieties of completely simple semigroups with subgroups in $\mathcal{A}_{p} \circ \mathcal{A}_{q}$ mentioned lastly in the previous paragraph is, in fact, a result concerning varieties of ordinary semigroups (without any additional unary operation).

M. Petrich and N.R. Reilly have completely described in [7] the lattice of all varieties of central completely simple semigroups in terms of the lattice of all varieties of groups. In fact, they have decomposed the former lattice into a subdirect product of the lattice of varieties of rectangular bands, the lattice of all varieties of abelian groups and the lattice of all varieties of groups. This result can also be found in [8], VIII.8. It seems that, up to now, the variety of all central completely simple semigroups represents the largest variety of completely simple semigroups for which a complete description of the lattice of its subvarieties modulo group varieties is available. None the less, already in the introduction to chapter VIII in [8], the authors have expressed their conviction that the whole lattice of all varieties of completely simple semigroups should not be amenable to an easy treatment modulo the varieties of groups. The results obtained in the present paper which are sketched briefly in the preceding paragraphs seem to confirm once and for all the justice of this estimate of the authors of the monograph [8].

This paper begins in section 1 with a brief outline of the general theory of near varieties of idempotent generated completely regular semigroups. In section 2 , this theory is adapted to near varieties of idempotent generated completely simple semigroups, taking advantage of the classic Rees matrix representation of completely simple semigroups. Particular attention is paid to near varieties of idempotent generated completely simple semigroups containing all rectangular bands and technical 
devices are developed with the view of their application in the later sections of this paper. In section 3, for any distinct prime numbers $p, q$, certain finite groups from the Malcev product $\mathcal{A}_{p} \circ \mathcal{A}_{q}$ of the varieties of abelian groups mentioned above are exhibited which will be used in the last two sections of the paper. In section 4, as distinct from the group varieties $\mathcal{A}_{p} \circ \mathcal{A}_{q}$ which are finitely generated, the varieties of completely simple semigroups containing all completely simple semigroups with maximal subgroups from the given group variety $\mathcal{A}_{p} \circ \mathcal{A}_{q}$ are shown not to be generated by any finitely generated completely simple semigroup. Finally, in the concluding section 5 , the tools prepared so far are employed, for any distinct prime numbers $p, q$, to provide $2 \$_{0}$ near varieties of idempotent generated completely simple semigroups whose maximal subgroups belong to the group variety $\mathcal{A}_{p} \circ \mathcal{A}_{q}$, as promised above.

1. Near varieties of idempotent generated completely regular semigroups. For any semigroup $S$, we denote by $E(S)$ the set of all idempotents of $S$. If $S$ is a regular semigroup, then the subsemigroup of $S$ generated by $E(S)$, which is also called the core of $S$ and is usually denoted by $C(S)$, is fairly well known to be a regular semigroup again. Returning briefly to the notions recalled already in the introduction to this paper, we can now say that a regular semigroup $S$ is called idempotent generated if its core $C(S)$ is equal to the whole semigroup $S$. From the considerations in the introduction, it becomes apparent that this viewpoint can be applied also in the particular case of completely regular semigroups, even though completely regular semigroups are often considered as unary semigroups with the additional unary operation assigning to every element its inverse within the maximal subgroup containing this element. This is incidentally this point of view of completely regular semigroups that we will use in the rest of this paper. That is, from now on, we will treat completely regular semigroups as unary semigroups in the way already specified.

Recall also from the introduction to this paper that by a near variety of idempotent generated completely regular semigroups we mean any class $\mathcal{W}$ of idempotent generated completely regular semigroups such that $\mathcal{W}$ is closed under the formation of idempotent generated regular subdirect products of arbitrary collections of semigroups, under the formation of idempotent generated regular subsemigroups, and under the formation of arbitrary homomorphic images. Further comments on the concept of near varieties of idempotent generated completely regular semigroups can be found in the introduction.

For any class $\mathcal{C}$ of completely regular semigroups, we denote by

$\mathcal{I C}$ - the class of all idempotent generated members of $\mathcal{C}$,

$\langle\mathcal{C}\rangle$ - the variety of completely regular semigroups generated by the class $\mathcal{C}$,

$\widehat{\mathcal{C}}$ — the class of all completely regular semigroups whose cores belong to $\mathcal{C}$.

Straightforward arguments show that if $\mathcal{V}$ is a variety of completely regular semigroups, then $\mathcal{I V}$ is a near variety of idempotent generated completely regular semigroups. In the same manner, one can check directly that if $\mathcal{V}$ is a variety of completely regular semigroups, then $\widehat{\mathcal{V}}$ is a variety of completely regular semigroups again, and if $\mathcal{W}$ is a near variety of idempotent generated completely regular semigroups, then $\widehat{\mathcal{W}}$ is a variety of completely regular semigroups, as well. In addition, if $\mathcal{C}$ is any class consisting only of completely simple semigroups, then the same is true of the class $\widehat{\mathcal{C}}$. 
Let $\mathcal{C R}$ stand for the variety of all completely regular semigroups and let $\mathcal{C S}$ stand for the variety of all completely simple semigroups. Let $\mathcal{L}(\mathcal{C R})$ denote the lattice of all varieties of completely regular semigroups and let $\mathcal{L}(\mathcal{I C} \mathcal{R})$ denote the lattice of all near varieties of idempotent generated completely regular semigroups. Let the notations $\mathcal{L}(\mathcal{C S})$ and $\mathcal{L}(\mathcal{I C S})$ have analogous meanings relative to the class $\mathcal{C S}$ of all completely simple semigroups. Then the following holds.

THEOREM 1.1. The mapping

$$
\mathcal{L}(\mathcal{C R}) \rightarrow \mathcal{L}(\mathcal{I C} \mathcal{R})
$$

given by the formula

$$
\mathcal{V} \mapsto \mathcal{I V}
$$

for all varieties $\mathcal{V}$ of completely regular semigroups, is a complete lattice homomorphism of the lattice $\mathcal{L}(\mathcal{C R})$ onto the lattice $\mathcal{L}(\mathcal{I C R})$. It induces a complete lattice congruence on $\mathcal{L}(\mathcal{C R})$ such that, for every near variety $\mathcal{W}$ of idempotent generated completely regular semigroups, the congruence class that maps to $\mathcal{W}$ is the interval

$$
[\langle\mathcal{W}\rangle, \widehat{\mathcal{W}}]
$$

in $\mathcal{L}(\mathcal{C R})$. For every variety $\mathcal{V}$ of completely regular semigroups, the congruence class containing $\mathcal{V}$ is the interval

$$
[\langle\mathcal{I} \mathcal{V}\rangle, \widehat{\mathcal{V}}]
$$

in $\mathcal{L}(\mathcal{C R})$.

Proof. The statements in this theorem can be verified in a manner analogous to the respective statements in [6], Theorem 4.4 concerning the lattices $\mathcal{L}(\mathcal{C S})$ and $\mathcal{L}(\mathcal{I C S})$, with some necessary amendments, of course.

For any near variety $\mathcal{W}$ of idempotent generated completely regular semigroups, it is obvious that $\mathcal{I} \widehat{\mathcal{W}}=\mathcal{W}$, which means that the above mapping is surjective. For any variety $\mathcal{V}$ of completely regular semigroups, we have $\mathcal{I} \mathcal{V}=\mathcal{V} \cap \mathcal{I C R}$, which shows that the above mapping preserves intersections of arbitrary non-empty families of varieties of completely regular semigroups. So let $\left\{\mathcal{V}_{i} \mid i \in I\right\}$ be a non-empty family of varieties of completely regular semigroups, and consider the joins $\bigvee_{i \in I} \mathcal{V}_{i}$ in $\mathcal{L}(\mathcal{C R})$ and $\bigvee_{i \in I} \mathcal{I} \mathcal{V}_{i}$ in $\mathcal{L}(\mathcal{I C R})$. Immediately we obtain that $\bigvee_{i \in I} \mathcal{I} \mathcal{V}_{i} \subseteq \mathcal{I}\left(\bigvee_{i \in I} \mathcal{V}_{i}\right)$. On the other hand, straightforward arguments based on the facts that any semigroup in $\mathcal{I}\left(\bigvee_{i \in I} \mathcal{V}_{i}\right)$ is idempotent generated and, at the same time, it is a homomorphic image of a regular subsemigroup of a direct product of a family of semigroups selected from the varieties $\mathcal{V}_{i}$, for $i \in I$, eventually show that any semigroup in $\mathcal{I}\left(\bigvee_{i \in I} \mathcal{V}_{i}\right)$ actually belongs to $\bigvee_{i \in I} \mathcal{I} \mathcal{V}_{i}$, which entails that $\mathcal{I}\left(\bigvee_{i \in I} \mathcal{V}_{i}\right) \subseteq \bigvee_{i \in I} \mathcal{I} \mathcal{V}_{i}$ and equality prevails. Thus the above mapping preserves also joins of arbitrary non-empty families of varieties of completely regular semigroups. Altogether, the above mapping is a complete lattice homomorphism. Consequently, it induces a complete lattice congruence on $\mathcal{L}(\mathcal{C R})$ whose congruence classes are intervals in $\mathcal{L}(\mathcal{C R})$. The fact that these intervals are of the form given above is now obvious.

The operator on the lattice $\mathcal{L}(\mathcal{C R})$ assigning to every variety $\mathcal{V}$ of completely regular semigroups the variety $\widehat{\mathcal{V}}$ was studied by L. Polák in section 7 of his paper [9]. In section 8 of [9], among other things, several non-trivial intervals of the form $[\mathcal{V}, \widehat{\mathcal{V}}]$ 
in the lattice $\mathcal{L}(\mathcal{C R})$ are exhibited, from which it is evident that the intervals of the above form $[\langle\mathcal{I} \mathcal{V}\rangle, \widehat{\mathcal{V}}]$ in $\mathcal{L}(\mathcal{C} \mathcal{R})$ may be considerably large.

We proceed with a slight amendment of the definition of free idempotent generated objects in classes of idempotent generated completely regular semigroups, which notion was originally conceived in section 3 of [6] for classes of completely simple semigroups. Thus let $\mathcal{C}$ be a class of idempotent generated completely regular semigroups and let $X$ be a non-empty set. Then by a free idempotent generated object in $\mathcal{C}$ on $X$ we mean a semigroup $S \in \mathcal{C}$ together with a mapping $\iota: X \rightarrow E(S)$ such that, for any semigroup $T \in \mathcal{C}$ and any mapping $\vartheta: X \rightarrow E(T)$, there exists a unique homomorphism $\varphi: S \rightarrow T$ which, when composed with $\iota$, yields $\vartheta$. Clearly, such a free idempotent generated object $S$ in $\mathcal{C}$ on $X$ is unique, up to isomorphism, if it exists. In addition, if the class $\mathcal{C}$ is closed under taking idempotent generated regular subsemigroups and the mentioned object $S$ exists, then $S$, viewed as a unary semigroup, is generated by the set of idempotents $\iota(X)$.

In order to provide further information on near varieties of idempotent generated completely regular semigroups, we introduce the following notation. For any nonempty (preferably infinite) set $X$, we denote by

\section{$U(X)$ - the absolutely free unary semigroup on $X$.}

Remember that, as in [8], I.10, $U(X)$ can be constructed as the smallest subset of the absolutely free semigroup on the set $X \cup\left\{(,)^{-1}\right\}$, where (and $)^{-1}$ are two new distinct elements, having the following properties: $X$ is a subset of $U(X)$, whenever $u, v \in U(X)$, then also $u v \in U(X)$, and whenever $u \in U(X)$, then also $(u)^{-1} \in U(X)$. Then elements of $U(X)$ will be called words over $X$. Next we denote by

$$
I U(X) \text { - the unary subsemigroup of } U(X) \text { generated by the set }\left\{x(x)^{-1}: x \in X\right\} \text {. }
$$

Notice that then $I U(X)$ is, in fact, the absolutely free unary semigroup on the set $\left\{x(x)^{-1}: x \in X\right\}$.

Remember next that, in this context, by an identity over the set $X$ we mean any pair $u \bumpeq v$ of words $u, v \in U(X)$, and that such an identity $u \bumpeq v$ is said to be satisfied in a completely regular semigroup $S$ if, for any mapping $\vartheta: X \rightarrow S$, we have $\theta(u)=\theta(v)$ where $\theta: U(X) \rightarrow S$ is the homomorphism of unary semigroups extending $\vartheta$. Now, by a nearly restricted identity over the set $X$ we mean any pair $s \bumpeq t$ of words $s, t \in I U(X)$. Of course, viewed from this perspective, nearly restricted identities over $X$ represent merely a special case of the previous common identities over $X$. Next we wish to say what it means for a nearly restricted identity $s \bumpeq t$ to be satisfied in an idempotent generated completely regular semigroup $S$. In view of the previous notes, we may consider this to be already defined, if we treat $s \bumpeq t$ as an ordinary identity and $S$ simply as some completely regular semigroup. However, it can be easily seen that, in this particular case, this definition is equivalent to the following one. A nearly restricted identity $s \bumpeq t$ over $X$ is satisfied in an idempotent generated completely regular semigroup $S$ if, for any mapping $\pi:\left\{x(x)^{-1}: x \in X\right\} \rightarrow E(S)$, we have $\varpi(s)=$ $\varpi(t)$ where $\varpi: I U(X) \rightarrow S$ is the homomorphism of unary semigroups extending $\pi$.

For any class $\mathcal{C}$ of completely regular semigroups and for any non-empty set $X$, we denote by

$\rho(\mathcal{C}, X)$ - the set of all identities over $X$ that are satisfied in all semigroups of $\mathcal{C}$. 
Then $\rho(\mathcal{C}, X)$ can be treated as a binary relation on $U(X)$. In fact, $\rho(\mathcal{C}, X)$ is then a fully invariant congruence on $U(X)$. Recall that this means that $\rho(\mathcal{C}, X)$ is invariant under all endomorphisms of $U(X)$. Often we will write for brevity $\rho(\mathcal{C})$ instead of $\rho(\mathcal{C}, X)$.

Likewise, for any class $\mathcal{C}$ of idempotent generated completely regular semigroups and for any non-empty set $X$, we denote by

$\imath \rho(\mathcal{C}, X)$ - the set of all nearly restricted identities over $X$ that are satisfied in all semigroups of $\mathcal{C}$.

Again, $\imath \rho(\mathcal{C}, X)$ can be treated as a binary relation, this time on $I U(X)$. In fact, $\imath \rho(\mathcal{C}, X)$ is a congruence on $I U(X)$ and it is invariant under all endomorphisms of $I U(X)$ which arise as restrictions to $I U(X)$ of homomorphisms of $U(X)$ into $I U(X)$. Often we will write for brevity $\imath \rho(\mathcal{C})$ instead of $\imath \rho(\mathcal{C}, X)$. (Just to be safe, remember that here as well as before we have congruences and homomorphisms of unary semigroups in mind.)

The notes in the previous paragraph lead us to introduce the following notion. Let $X$ be a non-empty set. We say that a congruence $\eta$ on the unary semigroup $I U(X)$ is nearly invariant if, for any words $s, t \in I U(X)$ satisfying $s \eta t$ and for any homomorphism of unary semigroups $\psi: U(X) \rightarrow I U(X)$, we have also $\psi(s) \eta \psi(t)$. Then the conclusion in the previous paragraph can be restated in the following form. For any class $\mathcal{C}$ of idempotent generated completely regular semigroups, $\imath \rho(\mathcal{C}, X)$ is a nearly invariant congruence on $I U(X)$.

In particular, for any non-empty set $X, \imath \rho(\mathcal{I C} \mathcal{R}, X)$ is the nearly invariant congruence on $I U(X)$ stemming from the set of all nearly restricted identities over $X$ which are satisfied in all idempotent generated completely regular semigroups. Then it is easy to verify that, for any congruence $\eta$ on $I U(X)$ satisfying $\imath \rho(\mathcal{I C R}, X) \subseteq \eta$, it is the case that this congruence $\eta$ on $I U(X)$ is nearly invariant if and only if the congruence $\eta / \imath \rho(\mathcal{I C} \mathcal{R}, X)$ on $I U(X) / \imath \rho(\mathcal{I C R})$ is fully invariant. Notice that this quotient semigroup is completely regular and that it is idempotent generated.

According to the standard results from universal algebra, in any class $\mathcal{V}$ of completely regular semigroups which is closed under taking regular subsemigroups and direct products of arbitrary families of semigroups, there exists a free object on any non-empty set $X$, and it is isomorphic to the quotient semigroup $U(X) / \rho(\mathcal{V})$. We shall see that an analogous statement holds for classes of idempotent generated completely regular semigroups and for free idempotent generated objects in such classes:

THEOREM 1.2. In any class $\mathcal{W}$ of idempotent generated completely regular semigroups which is closed under taking idempotent generated regular subsemigroups and idempotent generated regular subdirect products of arbitrary families of semigroups, there exists a free idempotent generated object on any non-empty set $X$, and it is isomorphic to the quotient semigroup $I U(X) / \imath \rho(\mathcal{W})$.

Proof. Let $\left\{\eta_{i}: i \in I\right\}$ be the non-empty collection of all congruences on the unary semigroup $I U(X)$ such that the quotient unary semigroups $I U(X) / \eta_{i}$ are isomorphic to completely regular semigroups in $\mathcal{W}$ and, for all $x \in X$, the elements $x(x)^{-1} \eta_{i}$ of $I U(X) / \eta_{i}$ are idempotents. Put $\eta=\bigcap_{i \in I} \eta_{i}$ and consider the quotient semigroup $I U(X) / \eta$. Then the unary semigroup $I U(X) / \eta$ is isomorphic to a subdirect product of the unary semigroups $I U(X) / \eta_{i}$, for $i \in I$, and hence $I U(X) / \eta$ is isomorphic to a regular subdirect product of a non-empty collection of completely regular semigroups from $\mathcal{W}$. Thus $I U(X) / \eta$ itself is a completely regular semigroup and, viewed as a unary semigroup, it is generated by the set of idempotents $\left\{x(x)^{-1} \eta: x \in X\right\}$. Therefore $I U(X) / \eta$ is idempotent generated, and hence it is isomorphic to a semigroup in $\mathcal{W}$. An 
obvious modification of the standard universal algebraic arguments then shows that this isomorphic copy of $I U(X) / \eta$ in $\mathcal{W}$ is a free idempotent generated object on $X$ in $\mathcal{W}$ and that $\eta$ is precisely the nearly invariant congruence $\imath \rho(\mathcal{W}, X)$ on $I U(X)$.

Observe that if $\mathcal{V}$ is a class of completely regular semigroups which is closed under taking regular subsemigroups and direct products of arbitrary families of semigroups, then the class $\mathcal{I V}$ of all idempotent generated completely regular semigroups from $\mathcal{V}$ is closed under taking idempotent generated regular subsemigroups and idempotent generated regular subdirect products of arbitrary families of semigroups. Notice also that, for any nearly restricted identity $s \bumpeq t$ on a non-empty set $X$, it is clearly true that $s \bumpeq t$ is satisfied in all semigroups of $\mathcal{I} \mathcal{V}$ if and only if $s \bumpeq t$, considered as an ordinary identity, is satisfied in all semigroups of $\mathcal{V}$. Hence, in this situation, we immediately obtain the equality

$$
\imath \rho(\mathcal{I} \mathcal{V}, X)=\rho(\mathcal{V}, X) \cap(I U(X) \times I U(X)) .
$$

This means that the mapping

$$
I U(X) / \imath \rho(\mathcal{I V}) \rightarrow U(X) / \rho(\mathcal{V})
$$

given by the formula

$$
\operatorname{t} \rho(\mathcal{I} \mathcal{V}) \mapsto t \rho(\mathcal{V})
$$

for all words $t \in I U(X)$, is an embedding of the semigroup $I U(X) / \imath \rho(\mathcal{I V})$ into the semigroup $U(X) / \rho(\mathcal{V})$. In view of Theorem 1.2 and the paragraph preceding it, we may now summarize our present findings in the following form:

COROLlary 1.3. Let $\mathcal{V}$ be a class of completely regular semigroups closed under taking regular subsemigroups and direct products of arbitrary families of semigroups. Then, for any non-empty set $X$, there exists a free object in $\mathcal{V}$ on $X$ which is isomorphic to the quotient semigroup $U(X) / \rho(\mathcal{V})$, and there exists a free idempotent generated object in $\mathcal{I} \mathcal{V}$ on $X$ which is isomorphic to the quotient semigroup $I U(X) / \imath \rho(\mathcal{I V})$. Moreover, the semigroup $I U(X) / \imath \rho(\mathcal{I V})$ is then isomorphic to the unary subsemigroup of $U(X) / \rho(\mathcal{V})$ generated by the set of idempotents $\left\{x(x)^{-1} \rho(\mathcal{V}): x \in X\right\}$.

For any non-empty (again preferably infinite) set $X$ and for any binary relation $\Phi$ on $U(X)$ viewed as a set of identities over $X$, we denote by

$[\Phi]$ — the class of all completely regular semigroups in which all identities from $\Phi$ are satisfied.

Then $[\Phi]$ is a variety of completely regular semigroups. Furthermore, for any binary relation $\Psi$ on $I U(X)$ viewed as a set of nearly restricted identities over $X$, we denote by

$\llbracket \Psi \rrbracket$ - the class of all idempotent generated completely regular semigroups in which all nearly restricted identities from $\Psi$ are satisfied.

Then it is fairly easy to verify that $\llbracket \Psi \rrbracket$ is a near variety of idempotent generated completely regular semigroups.

Now, by the classical Birkhoff theorem, we know that a class $\mathcal{V}$ of completely regular semigroups is a variety if and only if there exists a set $\Phi$ of identities of the above form such that $\mathcal{V}=[\Phi]$. Moreover, if this is the case then, for any infinite set $X$, 
we have $\mathcal{V}=[\rho(\mathcal{V}, X)]$. We shall see that an analogous Birkhoff-type theorem holds for near varieties of idempotent generated completely regular semigroups and for sets of nearly restricted identities:

THEOREM 1.4. A class $\mathcal{W}$ of idempotent generated completely regular semigroups is a near variety if and only if there exists a set $\Psi$ of nearly restricted identities such that $\mathcal{W}=\llbracket \Psi \rrbracket$. Moreover, if this is the case then, for any infinite set $X$, we have $\mathcal{W}=$ $\llbracket \imath \rho(\mathcal{W}, X) \rrbracket$.

Proof. In view of the above notes, it remains to show that, for any near variety $\mathcal{W}$ of idempotent generated completely regular semigroups and for any infinite set $X$, we have $\imath \rho(\mathcal{W}, X) \rrbracket \subseteq \mathcal{W}$, since the reverse containment is obvious. However, if $S$ is any idempotent generated completely regular semigroup such that all nearly restricted identities in $\imath \rho(\mathcal{W}, X)$ are satisfied in $S$ then, since $X$ is an infinite set, for whichever non-empty set $Y$, all nearly restricted identities in $\imath \rho(\mathcal{W}, Y)$ are satisfied in $S$, as well. In particular, this holds for sets $Y$ whose cardinality is at least as large as that of the set of idempotents $E(S)$. Consequently, every surjection of the set $\left\{y(y)^{-1}: y \in Y\right\}$ onto the set $E(S)$ can be extended in a unique way to a homomorphism of the quotient semigroup $I U(Y) / \imath \rho(\mathcal{W})$ onto the semigroup $S$. Uniqueness here follows from the fact that the semigroup $I U(Y) / \imath \rho(\mathcal{W})$ is generated by the set $\left\{y(y)^{-1} \imath \rho(\mathcal{W}): y \in Y\right\}$, and the homomorphism thus gained is surjective since the completely regular semigroup $S$ is idempotent generated. Since, at the same time, $I U(Y) / \imath \rho(\mathcal{W})$ is the free idempotent generated object in $\mathcal{W}$ on $Y$ by Theorem 1.2, it hence follows that the semigroup $S$ belongs to $\mathcal{W}$, as well.

According to a classical result from universal algebra, for any infinite set $X$, the rules

$$
\mathcal{V} \mapsto \rho(\mathcal{V}, X) \quad \text { and } \quad \rho \mapsto[\rho]
$$

determine mutually inverse order reversing bijections between the lattice of all varieties $\mathcal{V}$ of completely regular semigroups and the lattice of all fully invariant congruences $\rho$ on $U(X)$ satisfying $\rho(\mathcal{C} \mathcal{R}, X) \subseteq \rho$. We conclude this section by showing that an analogous result holds also for near varieties of idempotent generated completely regular semigroups and for nearly invariant congruences on $I U(X)$ containing $\imath \rho(\mathcal{I C R}, X)$.

First notice that, for any non-empty set $X$ and for any nearly invariant congruence $\eta$ on $I U(X)$ satisfying $\imath \rho(\mathcal{I C} \mathcal{R}, X) \subseteq \eta$, every pair in $\eta$ (viewed as a nearly restricted identity) is satisfied in the idempotent generated completely regular semigroup $I U(X) / \eta$. This holds since the congruence $\eta$ on $I U(X)$ is nearly invariant. Consequently, the semigroup $I U(X) / \eta$ belongs to the near variety $\llbracket \eta$. This clearly entails the equality $\eta=\imath \rho(\llbracket \eta \rrbracket, X)$. From this note and from the equality in Theorem 1.4, we deduce the desired conclusion:

COROLlary 1.5. For any infinite set $X$, the rules

$$
\mathcal{W} \mapsto \imath \rho(\mathcal{W}, X) \quad \text { and } \quad \eta \mapsto \llbracket \eta \rrbracket
$$

determine mutually inverse order reversing bijections between the lattice of all near varieties $\mathcal{W}$ of idempotent generated completely regular semigroups and the lattice of all nearly invariant congruences $\eta$ on $I U(X)$ satisfying $\imath \rho(\mathcal{I C R}, X) \subseteq \eta$. 
2. Near varieties of idempotent generated completely simple semigroups. By the famous Rees-Suschkewitsch theorem, completely simple semigroups are precisely semigroups which are isomorphic to Rees matrix semigroups over groups. Recall that the latter semigroups are constructed as follows. Let $H$ be a group, let $J$ and $\Lambda$ be non-empty sets and let $Q: \Lambda \times J \rightarrow H$ be any mapping. The mapping $Q$ is treated as a $\Lambda \times J$ matrix over $H$, and for any $\lambda \in \Lambda$ and $j \in J$, the image of the pair $(\lambda, j)$ under $Q$ is usually denoted by $q_{\lambda j}$. Thus one can write $Q=\left(q_{\lambda j}\right)_{\lambda \in \Lambda, j \in J}$. Define a multiplication on the set $J \times H \times \Lambda$ by the formula

$$
(i, g, \lambda)(j, h, \mu)=\left(i, g q_{\lambda j} h, \mu\right)
$$

for all $i, j \in J, g, h \in H$ and $\lambda, \mu \in \Lambda$. Then the set $J \times H \times \Lambda$ together with this multiplication forms a semigroup which is denoted by $\mathcal{M}(J, H, \Lambda ; Q)$ and is called the Rees matrix semigroup over the group $G$. The matrix $Q$ is called the sandwich matrix of this Rees matrix semigroup. Since $\mathcal{M}(J, H, \Lambda ; Q)$ is a completely simple semigroup, there is the unary operation of inversion on it, which is given by the formula

$$
(j, h, \lambda)^{-1}=\left(j, q_{\lambda j}^{-1} h^{-1} q_{\lambda j}^{-1}, \lambda\right),
$$

for all $j \in J, h \in H$ and $\lambda \in \Lambda$.

The representation of any given completely simple semigroup $S$ by a Rees matrix semigroup $\mathcal{M}(J, H, \Lambda ; Q)$ is not unique, as a rule. More precisely, the set $J$ has always the same cardinality and the same concerns the set $\Lambda$, and the group $H$ is unique up to isomorphism. But the sandwich matrix $Q=\left(q_{\lambda j}\right)_{\lambda \in \Lambda, j \in J}$ may vary. If there exist elements $\varkappa \in \Lambda$ and $i \in J$ such that, for all $j \in J$ and $\lambda \in \Lambda$, one has $q_{\varkappa j}=1$ and $q_{\lambda i}=1$, where 1 is the identity of the group $H$, then the sandwich matrix $Q$ is said to be normalized at the pair $(\varkappa, i)$. Now the previous formulation of the Rees-Suschkewitsch theorem can be adjusted by remembering the familiar fact that every completely simple semigroup is isomorphic to a Rees matrix semigroup $\mathcal{M}(J, H, \Lambda ; Q)$ whose sandwich matrix $Q$ is normalized at some pair of elements from $\Lambda \times J$.

As mentioned already, the class $\mathcal{C S}$ of all completely simple semigroups forms a variety of completely regular semigroups. Hence, for every non-empty set $X$, there exists a free object in $\mathcal{C S}$ on $X$, that is, there exists a free completely simple semigroup on $X$. Next we recall from [8], VIII.2 the representation of this free completely simple semigroup on $X$ by a suitable Rees matrix semigroup with normalized sandwich matrix. Before we can do so, we have to introduce some notation.

Thus let $X$ be a non-empty set. Fix a distiguished element $z \in X$ and choose new elements $p_{x y}$, for all $x, y \in X-\{z\}$, distinct from each other and distinct from all elements of $X$. Denote by

$G$ - the absolutely free group on the set $X \cup\left\{p_{x y}: x, y \in X-\{z\}\right\}$.

Next put $p_{x z}=1$ and $p_{z y}=1$, for all $x, y \in X$, where 1 now stands for the identity of the group $G$, and consider the $X \times X$ matrix

$$
P=\left(p_{x y}\right)_{x, y \in X}
$$

Furthermore, for any word $u \in U(X)$, we denote by

$\mathrm{h}(u)$ - the head of $u$, that is, the element of $X$ occurring in $u$ first from the left,

$\mathrm{t}(u)$ - the tail of $u$, that is, the element of $X$ occurring in $u$ first from the right. 
Additionally, for any word $u \in U(X)$, we denote by $\mathrm{m}(u)$ the element of the above absolutely free group $G$ which is constructed inductively as follows:

$$
\begin{array}{lr}
\mathrm{m}(x)=x, & \text { for all } x \in X, \\
\mathrm{~m}(u v)=\mathrm{m}(u) p_{\mathrm{t}(u) \mathrm{h}(v)} \mathrm{m}(v), & \text { for all } u, v \in U(X), \\
\mathrm{m}\left((u)^{-1}\right)=p_{\mathrm{t}(u) \mathrm{h}(u)}^{-1} \mathrm{~m}(u)^{-1} p_{\mathrm{t}(u) \mathrm{h}(u)}^{-1}, & \text { for all } u \in U(X) .
\end{array}
$$

It can be easily seen that, in this way, the element $\mathrm{m}(u)$ of $G$ is well defined, for all words $u \in U(X)$. Then, according to [8], VIII.2, the following holds.

RESUlT 2.1. The mapping

$$
\zeta: U(X) \rightarrow \mathcal{M}(X, G, X ; P)
$$

given by the formula

$$
u \mapsto(\mathrm{h}(u), \mathrm{m}(u), \mathrm{t}(u)),
$$

for all $u \in U(X)$, is a surjective homomorphism of unary semigroups which induces the congruence $\rho(\mathcal{C S}, X)$ on $U(X)$. This gives rise to an isomorphism

$$
\xi: U(X) / \rho(\mathcal{C S}) \rightarrow \mathcal{M}(X, G, X ; P)
$$

of the free completely simple semigroup $U(X) / \rho(\mathcal{C S})$ onto the Rees matrix semigroup $\mathcal{M}(X, G, X ; P)$. Therefore the Rees matrix semigroup $\mathcal{M}(X, G, X ; P)$ together with the mapping $\iota: X \rightarrow \mathcal{M}(X, G, X ; P)$ given by the formula $x \mapsto(x, x, x)$, for all $x \in X$, is a free object in $\mathcal{C S}$ on $X$.

Pursuing the above considerations, we notice that the idempotents of the form $\zeta\left(x(x)^{-1}\right)$ in the Rees matrix semigroup $\mathcal{M}(X, G, X ; P)$ are exactly the idempotents $\left(x, p_{x x}^{-1}, x\right)$, for all $x \in X$. Since, for any $x, y \in X$, we have

$$
\begin{aligned}
\left(x, p_{x x}^{-1}, x\right) & \left(\left(y, p_{y y}^{-1}, y\right)\left(x, p_{x x}^{-1}, x\right)\right)^{-1}\left(y, p_{y y}^{-1}, y\right) \\
= & \left(x, p_{x x}^{-1}, x\right)\left(y, p_{y y}^{-1} p_{y x} p_{x x}^{-1}, x\right)^{-1}\left(y, p_{y y}^{-1}, y\right) \\
= & \left(x, p_{x x}^{-1}, x\right)\left(y, p_{x y}^{-1} p_{x x} p_{y x}^{-1} p_{y y} p_{x y}^{-1}, x\right)\left(y, p_{y y}^{-1}, y\right) \\
= & \left(x, p_{y x}^{-1}, y\right)
\end{aligned}
$$

which is again an idempotent of $\mathcal{M}(X, G, X ; P)$, we see that the unary subsemigroup of $\mathcal{M}(X, G, X ; P)$ generated by the idempotents $\left(x, p_{x x}^{-1}, x\right)$, for all $x \in X$, actually contains all idempotents of $\mathcal{M}(X, G, X ; P)$, and hence it coincides with the core of $\mathcal{M}(X, G, X ; P)$. In particular, for any $x, y \in X-\{z\}$, the idempotents $(z, 1, x)$ and $(y, 1, z)$ are contained in the mentioned unary subsemigroup, whence it follows that also the elements $(z, 1, x)(y, 1, z)=\left(z, p_{x y}, z\right)$ and $\left(z, p_{x y}, z\right)^{-1}=\left(z, p_{x y}^{-1}, z\right)$ belong to this unary subsemigroup. Consequently, for any reduced group word $w$ over the set $\left\{p_{x y}: x, y \in X-\{z\}\right\}$, we obtain that the element $(z, w, z)$ occurs in this unary subsemigroup. Therefore, denoting by

$\widehat{G}$ - the subgroup of $G$ generated by the elements $p_{x y}$, for all $x, y \in X-\{z\}$, that is, the absolutely free group on the set $\left\{p_{x y}: x, y \in X-\{z\}\right\}$, 
we come to the conclusion that the unary subsemigroup of $\mathcal{M}(X, G, X ; P)$ generated by the idempotents $\left(x, p_{x x}^{-1}, x\right)$, for all $x \in X$, which coincides with the core of $\mathcal{M}(X, G, X ; P)$, is just the Rees matrix semigroup $\mathcal{M}(X, \widehat{G}, X ; P)$.

Since the class $\mathcal{I C S}$ of all idempotent generated completely simple semigroups forms a near variety of idempotent generated completely regular semigroups, by Theorem 1.2, there exists a free idempotent generated object in $\mathcal{I C S}$ on the set $X$, that is, there exists a free idempotent generated completely simple semigroup on $X$. Now, since for every word $s \in I U(X)$, we have $\mathrm{m}(s) \in \widehat{G}$, in view of the conclusion made at the end of the previous paragraph, from Result 2.1 and Corollary 1.3 we deduce the following representation of the free idempotent generated completely simple semigroup on $X$ by a Rees matrix semigroup with normalized sandwich matrix.

COROLlary 2.2. The mapping

$$
\hat{\zeta}: I U(X) \rightarrow \mathcal{M}(X, \widehat{G}, X ; P)
$$

given by the formula

$$
s \mapsto(\mathrm{h}(s), \mathrm{m}(s), \mathrm{t}(s)),
$$

for all $s \in I U(X)$, is a surjective homomorphism of unary semigroups which induces the congruence $\imath \rho(\mathcal{I C S}, X)$ on $I U(X)$. This gives rise to an isomorphism

$$
\hat{\xi}: I U(X) / \imath \rho(\mathcal{I C S}) \rightarrow \mathcal{M}(X, \widehat{G}, X ; P)
$$

of the free idempotent generated completely simple semigroup $I U(X) / \imath \rho(\mathcal{I C S})$ onto the Rees matrix semigroup $\mathcal{M}(X, \widehat{G}, X ; P)$. Therefore the Rees matrix semigroup $\mathcal{M}(X, \widehat{G}, X ; P)$ together with the mapping $\varepsilon: X \rightarrow \mathcal{M}(X, \widehat{G}, X ; P)$ given by the formula $x \mapsto\left(x, p_{x x}^{-1}, x\right)$, for all $x \in X$, is a free idempotent generated object in $\mathcal{I C S}$ on $X$.

Now we are in a position to demonstrate that the near variety $\mathcal{I C S}$ of all idempotent generated completely simple semigroups is not closed under the formation of direct products of infinite families of semigroups. Assume that the set $X$ in the previous considerations is not one-element and take an element $x \in X-\{z\}$. Consider the direct product of an infinite sequence of copies of the Rees matrix semigroup $\mathcal{M}(X, \widehat{G}, X ; P)$ from the previous corollary. Consider the element of this direct product of the form

$$
\left(\left(z, p_{x x}, z\right),\left(z, p_{x x} p_{x x}, z\right),\left(z, p_{x x} p_{x x} p_{x x}, z\right), \ldots,(z, \underbrace{p_{x x} \cdots p_{x x}}_{n}, z), \ldots\right) .
$$

From the above description of idempotents in $\mathcal{M}(X, \widehat{G}, X ; P)$ it now becomes apparent that this element cannot be obtained as a product of any finite sequence of idempotents of the direct product of the mentioned infinite sequence of copies of $\mathcal{M}(X, \widehat{G}, X ; P)$. Therefore this direct product is not idempotent generated.

Assume henceforth that the set $X$ is infinite. Then the rules $\mathcal{V} \mapsto \rho(\mathcal{V}, X)$ and $\rho \mapsto[\rho]$ determine mutually inverse order reversing bijections between the lattice of all varieties $\mathcal{V}$ of completely simple semigroups and the lattice of all fully invariant congruences $\rho$ on $U(X)$ satisfying $\rho(\mathcal{C S}, X) \subseteq \rho$. Hence, in view of Result 2.1, we deduce that there is an order reversing bijection between the lattice of all varieties of completely simple semigroups and the lattice of all fully invariant congruences on the Rees matrix semigroup $\mathcal{M}(X, G, X ; P)$. We further confine our attention to 
varieties of completely simple semigroups containing all rectangular bands. Let $\mathcal{R B}$ stand for the variety of all rectangular bands. Since the Green's relation $\mathcal{H}$ is the largest idempotent separating congruence on $\mathcal{M}(X, G, X ; P)$ and the quotient semigroup $\mathcal{M}(X, G, X ; P) / \mathcal{H}$ is clearly the free rectangular band on $X$, the previously mentioned bijection restricts to give an order reversing bijection between the interval consisting of all varieties $\mathcal{V}$ of completely simple semigroups satisfying $\mathcal{R B} \subseteq \mathcal{V}$ and the lattice of all idempotent separating fully invariant congruences on $\mathcal{M}(X, G, X ; P)$.

Since we have to deal with congruences of this kind, we first recall from [8], III.4 the characterization of idempotent separating congruences on arbitrary Rees matrix semigroups.

Result 2.3. Let $\mathcal{M}(J, H, \Lambda ; Q)$ be a Rees matrix semigroup. Then the mapping assigning to every normal subgroup $N$ of the group $H$ the binary relation $\rho_{N}$ on the set $J \times H \times \Lambda$ defined by the formula

$$
(i, g, \lambda) \rho_{N}(j, h, \mu) \Leftrightarrow i=j, g h^{-1} \in N, \lambda=\mu,
$$

for all $i, j \in J, g, h \in H$ and $\lambda, \mu \in \Lambda$, is an isomorphism of the lattice of all normal subgroups of $H$ onto the lattice of all idempotent separating congruences on $\mathcal{M}(J, H, \Lambda ; Q)$. Moreover, the quotient semigroup $\mathcal{M}(J, H, \Lambda ; Q) / \rho_{N}$ is isomorphic to the Rees matrix semigroup $\mathcal{M}(J, H / N, \Lambda ; Q / N)$ where $Q / N$ is the $\Lambda \times J$ matrix over $H / N$ of the form $Q / N=\left(q_{\lambda j} N\right)_{\lambda \in \Lambda, j \in J}$.

Next, since we have to deal with fully invariant congruences, we recall from [8], III. 3 the description of all endomorphisms of arbitrary Rees matrix semigroups with normalized sandwich matrices.

RESUlt 2.4. Let $\mathcal{M}(J, H, \Lambda ; Q)$ be a Rees matrix semigroup whose sandwich matrix $Q$ is normalized at some pair $(\varkappa, i) \in \Lambda \times J$. Let $\sigma: I \rightarrow I$ and $\tau: \Lambda \rightarrow \Lambda$ be any mappings and let $\omega: H \rightarrow H$ be any endomorphism such that

$$
\omega\left(q_{\lambda j}\right)=q_{\tau(\varkappa) \sigma(i)} q_{\tau(\lambda) \sigma(i)}^{-1} q_{\tau(\lambda) \sigma(j)} q_{\tau(\varkappa) \sigma(j)}^{-1}
$$

holds for all $j \in J$ and $\lambda \in \Lambda$. (This condition obviously holds if $j=i$ or $\lambda=\varkappa$.) Then the mapping of the set $J \times H \times \Lambda$ into itself defined by the formula

$$
(j, h, \lambda) \mapsto\left(\sigma(j), q_{\tau(\varkappa) \sigma(j)}^{-1} \omega(h) q_{\tau(\varkappa) \sigma(i)} q_{\tau(\lambda) \sigma(i)}^{-1}, \tau(\lambda)\right),
$$

for all $j \in J, h \in H$ and $\lambda \in \Lambda$, is an endomorphism of $\mathcal{M}(J, H, \Lambda ; Q)$, and conversely, every endomorphism of $\mathcal{M}(J, H, \Lambda ; Q)$ can so be written uniquely.

From these two results the following conclusion follows essentially immediately.

Corollary 2.5. Let $\mathcal{M}(J, H, \Lambda ; Q)$ be a Rees matrix semigroup whose sandwich matrix $Q$ is normalized at some pair $(\varkappa, i) \in \Lambda \times J$. Then, for any normal subgroup $N$ of $H$, the idempotent separating congruence $\rho_{N}$ on $\mathcal{M}(J, H, \Lambda ; Q)$ corresponding to $N$ according to Result 2.3 is fully invariant if and only if the condition $\omega(N) \subseteq N$ holds for all endomorphisms $\omega$ of $H$ that arise in association with endomorphisms of $\mathcal{M}(J, H, \Lambda ; Q)$, as described in Result 2.4.

Remember that by Result 2.1, the Rees matrix semigroup $\mathcal{M}(X, G, X ; P)$ is a free completely simple semigroup on $X$. Notice that its sandwich matrix $P$ is normalized at the pair $(z, z)$ where $z \in X$ is the distinguished element. If the set $X$ is infinite then, from 
the text preceding Result 2.3, we know that there is a bijection between the sublattice of all varieties $\mathcal{V}$ of completely simple semigroups satisfying $\mathcal{R B} \subseteq \mathcal{V}$ and the lattice of all idempotent separating fully invariant congruences on $\mathcal{M}(X, G, X ; P)$. Now, applying Corollary 2.5 to the Rees matrix semigroup $\mathcal{M}(X, G, X ; P)$, we get a description of all idempotent separating fully invariant congruences on $\mathcal{M}(X, G, X ; P)$. This was incidentally done in [8], VIII.3. Thus we come to the conclusion that there is a bijection between the sublattice of all varieties $\mathcal{V}$ of completely simple semigroups satisfying $\mathcal{R B} \subseteq \mathcal{V}$ and the lattice of all normal subgroups $N$ of $G$ satisfying $\omega(N) \subseteq N$ for all endomorphisms $\omega$ of $G$ that arise in association with endomorphisms of $\mathcal{M}(X, G, X ; P)$. For any variety $\mathcal{V}$ of completely simple semigroups satisfying $\mathcal{R} \mathcal{B} \subseteq \mathcal{V}$, we denote by

$N_{\mathcal{V}}$ - the normal subgroup of $G$ corresponding to $\mathcal{V}$ in the way just described.

Then from Results 2.1 and 2.3 we obtain the following representation of the free semigroup on $X$ in any variety $\mathcal{V}$ of completely simple semigroups satisfying $\mathcal{R B} \subseteq \mathcal{V}$ by a Rees matrix semigroup with normalized sandwich matrix.

COROLlARY 2.6. Let $\mathcal{V}$ be any variety of completely simple semigroups satisfying $\mathcal{R B} \subseteq \mathcal{V}$. Then the mapping

$$
\zeta_{\mathcal{V}}: U(X) \rightarrow \mathcal{M}\left(X, G / N_{\mathcal{V}}, X ; P / N_{\mathcal{V}}\right)
$$

given by the formula

$$
u \mapsto\left(\mathrm{h}(u), \mathrm{m}(u) N_{\mathcal{V}}, \mathrm{t}(u)\right),
$$

for all $u \in U(X)$, is a surjective homomorphism of unary semigroups which induces the congruence $\rho(\mathcal{V}, X)$ on $U(X)$. This gives rise to an isomorphism

$$
\xi_{\mathcal{V}}: U(X) / \rho(\mathcal{V}) \rightarrow \mathcal{M}\left(X, G / N_{\mathcal{V}}, X ; P / N_{\mathcal{V}}\right)
$$

of the free semigroup $U(X) / \rho(\mathcal{V})$ in the variety $\mathcal{V}$ on $X$ onto the Rees matrix semigroup $\mathcal{M}\left(X, G / N_{\mathcal{V}}, X ; P / N_{\mathcal{V}}\right)$. Therefore $\mathcal{M}\left(X, G / N_{\mathcal{V}}, X ; P / N_{\mathcal{V}}\right)$ is a free semigroup in $\mathcal{V}$ on $X$.

Remember once again our assumption, that the set $X$ is infinite. Now consider the near varieties of idempotent generated completely simple semigroups. Note that the class $\mathcal{R B}$ of all rectangular bands is itself also a near variety of idempotent generated completely simple semigroups. Furthermore, just as in the previous section, one can check that, for any congruence $\delta$ on $I U(X)$ satisfying $\imath \rho(\mathcal{I C S}, X) \subseteq \delta$, it is true that this congruence $\delta$ on $I U(X)$ is nearly invariant if and only if the congruence $\delta / \imath \rho(\mathcal{I C S}, X)$ on $I U(X) / \imath \rho(\mathcal{I C S})$ is fully invariant. Then from Corollaries 1.5 and 2.2 we obtain an order reversing bijection between the lattice of all near varieties of idempotent generated completely simple semigroups and the lattice of all fully invariant congruences on the Rees matrix semigroup $\mathcal{M}(X, \widehat{G}, X ; P)$. We next again confine our attention to those near varieties of idempotent generated completely simple semigroups which contain all rectangular bands. Arguing in the same way as earlier in this section, we conclude that the bijection just mentioned restricts to give an order reversing bijection between the interval consisting of all near varieties $\mathcal{W}$ of idempotent generated completely simple semigroups satisfying $\mathcal{R B} \subseteq \mathcal{W}$ and the lattice of all idempotent separating fully invariant congruences on $\mathcal{M}(\bar{X}, \widehat{G}, X ; P)$. Recall also once again that 
the sandwich matrix $P$ is normalized at the pair $(z, z)$. Thus we can apply Corollary 2.5 to the Rees matrix semigroup $\mathcal{M}(X, \widehat{G}, X ; P)$ to get a description of all idempotent separating fully invariant congruences on $\mathcal{M}(X, \widehat{G}, X ; P)$. In this way, we come to the conclusion that there is a bijection between the sublattice of all near varieties varieties $\mathcal{W}$ of idempotent generated completely simple semigroups satisfying $\mathcal{R B} \subseteq \mathcal{W}$ and the lattice of all normal subgroups $M$ of $\widehat{G}$ satisfying $\omega(M) \subseteq M$ for all endomorphisms $\omega$ of $\widehat{G}$ that arise in association with endomorphisms of $\mathcal{M}(X, \widehat{G}, X ; P)$. For any near variety $\mathcal{W}$ of idempotent generated completely simple semigroups satisfying $\mathcal{R} \mathcal{B} \subseteq \mathcal{W}$, we denote by

$M_{\mathcal{W}}$ - the normal subgroup of $\widehat{G}$ corresponding to $\mathcal{W}$ in the way just described.

Then from Corollary 2.2 and Result 2.3 we obtain the following representation of the free idempotent generated semigroup on $X$ in any near variety $\mathcal{W}$ of idempotent generated completely simple semigroups satisfying $\mathcal{R B} \subseteq \mathcal{W}$ by a Rees matrix semigroup with normalized sandwich matrix.

COROLlary 2.7. Let $\mathcal{W}$ be any near variety of idempotent generated completely simple semigroups satisfying $\mathcal{R B} \subseteq \mathcal{W}$. Then the mapping

$$
\hat{\zeta}_{\mathcal{W}}: I U(X) \rightarrow \mathcal{M}\left(X, \widehat{G} / M_{\mathcal{W}}, X ; P / M_{\mathcal{W}}\right)
$$

given by the formula

$$
s \mapsto\left(\mathrm{h}(s), \mathrm{m}(s) M_{\mathcal{W}}, \mathrm{t}(s)\right),
$$

for all $s \in I U(X)$, is a surjective homomorphism of unary semigroups which induces the congruence $\imath \rho(\mathcal{W}, X)$ on $I U(X)$. This gives rise to an isomorphism

$$
\hat{\xi}_{\mathcal{W}}: I U(X) / \imath \rho(\mathcal{W}) \rightarrow \mathcal{M}\left(X, \widehat{G} / M_{\mathcal{W}}, X ; P / M_{\mathcal{W}}\right)
$$

of the free idempotent generated semigroup $I U(X) / \imath \rho(\mathcal{W})$ in $\mathcal{W}$ on $X$ onto the Rees matrix semigroup $\mathcal{M}\left(X, \widehat{G} / M_{\mathcal{W}}, X ; P / M_{\mathcal{W}}\right)$. Therefore $\mathcal{M}\left(X, \widehat{G} / M_{\mathcal{W}}, X ; P / M_{\mathcal{W}}\right)$ is a free idempotent generated semigroup in $\mathcal{W}$ on $X$.

REMARK. According to Result 2.4, the normal subgroups $M$ of $\widehat{G}$ appearing in the text preceding the above corollary are exactly those normal subgroups which are invariant under all endomorphisms $\omega$ of $\widehat{G}$ satisfying the condition that, for some two mappings $\sigma, \tau: X \rightarrow X$, the eqality

$$
\omega\left(p_{x y}\right)=p_{\tau(z) \sigma(z)} p_{\tau(x) \sigma(z)}^{-1} p_{\tau(x) \sigma(y)} p_{\tau(z) \sigma(y)}^{-1}
$$

holds for all $x, y \in X$. (This equality obviously holds if $x=z$ or $y=z$.) Since $\widehat{G}$ is the absolutely free group on the set $\left\{p_{x y}: x, y \in X-\{z\}\right\}$, the mentioned endomorphisms $\omega$ of $\widehat{G}$ are fully determined by the underlying mappings $\sigma, \tau: X \rightarrow X$, which, in turn, can be chosen arbitrarily.

Now let $\mathcal{V}$ be any variety of completely simple semigroups satisfying $\mathcal{R B} \subseteq \mathcal{V}$. Then the near variety $\mathcal{I} \mathcal{V}$ of idempotent generated completely simple semigroups likewise satisfies $\mathcal{R B} \subseteq \mathcal{I} \mathcal{V}$. By Corollary 1.3, the free idempotent generated semigroup $I U(X) / \imath \rho(\mathcal{I V})$ in $\mathcal{I V}$ on $X$ is isomorphic to the unary subsemigroup of the free semigroup $U(X) / \rho(\mathcal{V})$ in $\mathcal{V}$ on $X$ which is generated by the set of idempotents $\left\{x(x)^{-1} \rho(\mathcal{V}): x \in X\right\}$. Consequently, by Corollaries 2.6 and 2.7, the idempotent 
generated Rees matrix semigroup $\mathcal{M}\left(X, \widehat{G} / M_{\mathcal{I V}}, X ; P / M_{\mathcal{I V}}\right)$ is isomorphic to the unary subsemigroup of the Rees matrix semigroup $\mathcal{M}\left(X, G / N_{\mathcal{V}}, X ; P / N_{\mathcal{V}}\right)$ generated by the set of idempotents $\left\{\left(x, p_{x x}^{-1} N_{\mathcal{V}}, x\right): x \in X\right\}$. From the notes following Result 2.1 it becomes apparent that the mentioned unary subsemigroup is actually the core of the semigroup $\mathcal{M}\left(X, G / N_{\mathcal{V}}, X ; P / N_{\mathcal{V}}\right)$ and that it is, in fact, the Rees matrix semigroup $\mathcal{M}\left(X, \widehat{G} / \widehat{N}_{\mathcal{V}}, X ; P / \widehat{N}_{\mathcal{V}}\right)$ where $\widehat{N}_{\mathcal{V}}=\widehat{G} \cap N_{\mathcal{V}}$. Comparing this with the former idempotent generated Rees matrix semigroup, we thus come to the equality $M_{\mathcal{I V}}=$ $\widehat{G} \cap N_{\mathcal{V}}$. Thus we arrive at the following conclusion which, with some shortcomings though, was also obtained in [6], Theorem 3.2.

COROLLARY 2.8. Let $\mathcal{V}$ be any variety of completely simple semigroups satisfying $\mathcal{R B} \subseteq \mathcal{V}$. Then the Rees matrix semigroup $\mathcal{M}\left(X, \widehat{G} / \widehat{N}_{\mathcal{V}}, X ; P / \widehat{N}_{\mathcal{V}}\right)$ where $\widehat{N}_{\mathcal{V}}=\widehat{G} \cap N_{\mathcal{V}}$ together with the mapping assigning to every element $x \in X$ the idempotent $\left(x, p_{x x}^{-1} \widehat{N}_{\mathcal{V}}, x\right)$ is a free idempotent generated object on $X$ in the near variety $\mathcal{I} \mathcal{V}$.

We next adjust the Birkhoff theorem to the varieties of completely simple semigroups which contain all rectangular bands. In this connection, we introduce the following notation which will prove useful later in this paper. We continue working with our infinite set $X$ containing the distinguished element $z \in X$.

We begin with the following rules assigning a word from $U(X)$ to every element of the set $X \cup\left\{p_{x y}: x, y \in X-\{z\}\right\}$ of generators of the absolutely free group $G$ and also to the inverse in $G$ of each of these elements :

$$
\begin{array}{rlrl}
x & \mapsto z(z)^{-1} x z(z)^{-1}, & \text { for all } x \in X, \\
x^{-1} & \mapsto\left(z(z)^{-1} x z(z)^{-1}\right)^{-1}, \quad \text { for all } x \in X, \\
p_{x y} & \mapsto\left(z(z)^{-1} x(x)^{-1}\right)^{-1} z(z)^{-1} x(x)^{-1} y(y)^{-1} z(z)^{-1}\left(y(y)^{-1} z(z)^{-1}\right)^{-1} \\
& \text { for all } x, y \in X-\{z\}, \\
p_{x y}^{-1} & \mapsto\left(\left(z(z)^{-1} x(x)^{-1}\right)^{-1} z(z)^{-1} x(x)^{-1} y(y)^{-1} z(z)^{-1}\left(y(y)^{-1} z(z)^{-1}\right)^{-1}\right)^{-1} \\
& \text { for all } x, y \in X-\{z\} .
\end{array}
$$

These assignments can be extended in a unique way to a homomorphism $\gamma$ of the free semigroup on the set $X \cup\left\{x^{-1}: x \in X\right\} \cup\left\{p_{x y}, p_{x y}^{-1}: x, y \in X-\{z\}\right\}$ to the semigroup $U(X)$. In particular, every element $g \in G-\{1\}$ expressed as a reduced group word over the set $X \cup\left\{p_{x y}: x, y \in X-\{z\}\right\}$ can be viewed as an element of the former free semigroup, and hence, for every element $g \in G-\{1\}$, the word $\gamma(g)$ of $U(X)$ is defined. Then it can be verified straightforwardly that, for every element $g \in G-\{1\}$, we have $\mathrm{m}(\gamma(g))=g$. It is also worth noting that, for every element $h \in \widehat{G}-\{1\}$, the word $\gamma(h)$ clearly belongs to the subsemigroup $I U(X)$.

Proposition 2.9. A class $\mathcal{V}$ of completely regular semigroups is a variety of completely simple semigroups satisfying $\mathcal{R B} \subseteq \mathcal{V}$ if and only if there exists a set $\Phi$ of identities of the form $u^{2} \bumpeq u$ where $u \in U(X)$ has the property that $\mathrm{h}(u)=z=\mathrm{t}(u)$ such that $\mathcal{V}=\mathcal{C S} \cap[\Phi]$. Moreover, if this is the case then $\mathcal{V}=\mathcal{C S} \cap[\widetilde{\Phi}]$ where $\widetilde{\Phi}=$ $\rho(\mathcal{V}, X) \cap\left\{\left(u^{2}, u\right): u \in U(X), \mathrm{h}(u)=z=\mathrm{t}(u)\right\}$.

Proof. Every rectangular band satisfies all identities of the form $u^{2} \bumpeq u$ where $u \in U(X)$ is such that $\mathrm{h}(u)=z=\mathrm{t}(u)$. Consequently, every class $\mathcal{V}$ of completely regular semigroups of the form $\mathcal{V}=\mathcal{C S} \cap[\Phi]$ where $\Phi$ is a set of identities of the mentioned kind is a variety of completely simple semigroups such that $\mathcal{R B} \subseteq \mathcal{V}$. 
Conversely, let $\mathcal{V}$ be a variety of completely simple semigroups satisfying $\mathcal{R B} \subseteq \mathcal{V}$. Consider any element $r \in N_{\mathcal{V}}-\{1\}$ and the corresponding word $\gamma(r)$ of $U(X)$. Since $\mathrm{h}(\gamma(r))=z=\mathrm{t}(\gamma(r))$, we obtain $\mathrm{m}\left(\gamma\left(r^{2}\right)\right)=\mathrm{m}(\gamma(r))^{2}=r^{2}$, and hence $\mathrm{m}\left(\gamma\left(r^{2}\right)\right) N_{\mathcal{V}}=r^{2} N_{\mathcal{V}}=N_{\mathcal{V}}=r N_{\mathcal{V}}=\mathrm{m}(\gamma(r)) N_{\mathcal{V}}$. Consequently, by Corollary 2.6, we have $\gamma(r)^{2} \rho(\mathcal{V}, X) \gamma(r)$, which means that the identity $\gamma(r)^{2} \bumpeq \gamma(r)$ is satisfied in $\mathcal{V}$. Therefore, putting $\mathcal{U}=\mathcal{C S} \cap\left[\left\{\gamma(r)^{2} \bumpeq \gamma(r): r \in N_{\mathcal{V}}-\{1\}\right\}\right]$, we have $\mathcal{V} \subseteq \mathcal{U}$. On the other hand, for every element $r \in N_{\mathcal{V}}-\{1\}$, we now have $\gamma(r)^{2} \rho(\mathcal{U}, X) \gamma(r)$, whence, by Corollary 2.6 again, we obtain $\mathrm{m}\left(\gamma\left(r^{2}\right)\right) N_{\mathcal{U}}=\mathrm{m}(\gamma(r)) N_{\mathcal{U}}$, that is, $r^{2} N_{\mathcal{U}}=r N_{\mathcal{U}}$, which entails that $r \in N_{\mathcal{U}}-\{1\}$. This shows that $N_{\mathcal{V}} \subseteq N_{\mathcal{U}}$, and so $\mathcal{U} \subseteq \mathcal{V}$. Altogether we get that $\mathcal{V}=\mathcal{U}$, so that the variety $\mathcal{V}$ is determined within $\mathcal{C S}$ by identities of the required form. The last statement of this proposition is now obvious.

We can similarly adapt the Birkhoff-type theorem for near varieties of idempotent generated completely regular semigroups to the near varieties of idempotent generated completely simple semigroups containing all rectangular bands.

Proposition 2.10. A class $\mathcal{W}$ of idempotent generated completely regular semigroups is a near variety of idempotent generated completely simple semigroups satisfying $\mathcal{R B} \subseteq \mathcal{W}$ if and only if there exists a set $\Psi$ of nearly restricted identities of type $v^{2} \bumpeq v$ where $v \in I U(X)$ has the property that $\mathrm{h}(v)=z=\mathrm{t}(v)$ such that $\mathcal{W}=\mathcal{I C S} \cap \llbracket \Psi \rrbracket$. Moreover, if this is the case then $\mathcal{W}=\mathcal{I C S} \cap \llbracket \widetilde{\Psi} \rrbracket$ where

$$
\widetilde{\Psi}=\imath \rho(\mathcal{W}, X) \cap\left\{\left(v^{2}, v\right): v \in I U(X), \mathrm{h}(v)=z=\mathrm{t}(v)\right\} .
$$

Proof. This proposition can be proved in the same manner as the previous one, with the help of Theorem 1.4, of course. Elements $r \in M_{\mathcal{W}}-\{1\}$ and the corresponding words $\gamma(r)$ of $I U(X)$ are considered now and Corollary 2.7 is invoked in this case in order to complete the arguments.

The last two propositions indicate that it will be useful to explore in more detail what it means to say that an identity of the form $u^{2} \bumpeq u$, where $u \in U(X)$ is such that $\mathrm{h}(u)=z=\mathrm{t}(u)$, is satisfied in a completely simple semigroup, that is, in a Rees matrix semigroup, say $\mathcal{M}(J, H, \Lambda ; Q)$. For this purpose, we will need the procedure of normalization of the sandwich matrix $Q$ described in [8], III.3 which produces a Rees matrix representation of $\mathcal{M}(J, H, \Lambda ; Q)$ whose sandwich matrix is normalized at any position selected beforehand. This procedure looks as follows.

Thus, once again, let $\mathcal{M}(J, H, \Lambda ; Q)$ be an arbitrary Rees matrix semigroup where $Q=\left(q_{\lambda j}\right)_{\lambda \in \Lambda, j \in J}$. Select arbitrary elements $\varkappa \in \Lambda$ and $i \in J$ and consider the Rees matrix semigroup $\mathcal{M}\left(J, H, \Lambda ; \bar{Q}^{\varkappa i}\right)$ whose sandwich matrix $\bar{Q}^{\varkappa i}$ is of the form

$$
\bar{Q}^{\varkappa i}=\left(q_{\lambda i}^{-1} q_{\lambda j} q_{\varkappa j}^{-1} q_{\varkappa i}\right)_{\lambda \in \Lambda, j \in J}
$$

Then the matrix $\bar{Q}^{\varkappa i}$ is obviously normalized at the selected pair $(\varkappa, i)$. Moreover, the mapping

$$
\varsigma_{\varkappa i}: J \times H \times \Lambda \rightarrow J \times H \times \Lambda
$$

given by the formula

$$
(j, h, \lambda) \mapsto\left(j, q_{\varkappa i}^{-1} q_{\varkappa j} h q_{\lambda i}, \lambda\right),
$$

for all $j \in J, h \in H$ and $\lambda \in \Lambda$, is an isomorphism of the $\operatorname{semigroup} \mathcal{M}(J, H, \Lambda ; Q)$ onto the semigroup $\mathcal{M}\left(J, H, \Lambda ; \bar{Q}^{\varkappa i}\right)$. This statement can be verified straightforwardly. 
We continue by introducing, for the selected elements $\varkappa \in \Lambda$ and $i \in J$ and for any mapping $\vartheta: X \rightarrow J \times H \times \Lambda$ satisfying $\vartheta(z) \in\{i\} \times H \times\{\varkappa\}$, a homomorphism

$$
\chi_{\varkappa i}^{\vartheta}: G \rightarrow H
$$

of the absolutely free group $G$ on the set $X \cup\left\{p_{x y}: x, y \in X-\{z\}\right\}$ into the group $H$ in the following way. Clearly, it suffices to determine this homomorphism only on the generators of $G$. For this purpose, consider first the mappings $\varphi: X \rightarrow J, \beta: X \rightarrow H$ and $\psi: X \rightarrow \Lambda$ given, for every $x \in X$, by the formula $\vartheta(x)=(\varphi(x), \beta(x), \psi(x))$. Then define the homomorphism $\chi_{\varkappa i}^{\vartheta}$ by the following assignments :

$$
\begin{aligned}
x & \mapsto \beta(x), & \text { for all } x \in X, \\
p_{x y} & \mapsto q_{\psi(x) i}^{-1} q_{\psi(x) \varphi(y)} q_{\varkappa \varphi(y)}^{-1} q_{\varkappa i}, & \text { for all } x, y \in X-\{z\} .
\end{aligned}
$$

Note that in the second set of assignments, we could include the same assignments also for $x=z$ or $y=z$, since these added assignments reduce to $1 \mapsto 1$ in view of the fact that $\varphi(z)=i$ and $\psi(z)=\varkappa$. Observe also that then, for any $x, y \in X$, the homomorphism $\chi_{\varkappa i}^{\vartheta}$ sends the element $p_{x y}$ to the entry of the matrix $\bar{Q}$ occurring in the position $(\psi(x), \varphi(y))$.

Now we are ready to state and prove the following criterion for the validity of the above-mentioned identities in arbitrary Rees matrix semigroups:

Proposition 2.11. Let $\mathcal{M}(J, H, \Lambda ; Q)$ be a Rees matrix semigroup. Let $u \in U(X)$ be any word such that $\mathrm{h}(u)=z=\mathrm{t}(u)$. Then the identity $u^{2} \bumpeq u$ is satisfied in the semigroup $\mathcal{M}(J, H, \Lambda ; Q)$ if and only if, for every $i \in J, \varkappa \in \Lambda$ and for every mapping $\vartheta: X \rightarrow$ $J \times H \times \Lambda$ such that $\vartheta(z) \in\{i\} \times H \times\{\varkappa\}$, the equality $\chi_{\varkappa i}^{\vartheta}(\mathrm{m}(u))=1$ holds in $H$.

Proof. Remember that, by Result 2.1, the semigroup $\mathcal{M}(X, G, X ; P)$ together with the mapping $\iota: X \rightarrow \mathcal{M}(X, G, X ; P)$ given, for every $x \in X$, by the formula $x \mapsto(x, x, x)$ is a free completely simple semigroup on $X$. The mapping $\iota$ extends in a unique way to a homomorphism of unary semigroups $\zeta: U(X) \rightarrow \mathcal{M}(X, G, X ; P)$.

Now, by definition, the identity $u^{2} \bumpeq u$ is satisfied in $\mathcal{M}(J, H, \Lambda ; Q)$ if, for every mapping $\eta: X \rightarrow J \times H \times \Lambda$, we have $\theta\left(\zeta\left(u^{2}\right)\right)=\theta(\zeta(u))$ where $\theta: \mathcal{M}(X, G, X ; P) \rightarrow$ $\mathcal{M}(J, H, \Lambda ; Q)$ is the homomorphism satisfying $\theta(x, x, x)=\eta(x)$, for all $x \in X$, that is, $\theta$ is the homomorphism which, when composed with $\iota$, yields $\eta$. Now let us take care of the element $\eta(z)$. Of course, $\eta(z)=(i, h, \varkappa)$ for some $i \in J, h \in H$ and $\varkappa \in \Lambda$, and so $\eta(z) \in\{i\} \times H \times\{\varkappa\}$ for these $i \in J$ and $\varkappa \in \Lambda$. As $\eta: X \rightarrow J \times H \times \Lambda$ is an arbitrary mapping, the mentioned elements $i \in J$ and $\varkappa \in \Lambda$ can also be arbitrary. Having in view the above isomorphism $\varsigma_{\varkappa i}: \mathcal{M}(J, H, \Lambda ; Q) \rightarrow \mathcal{M}\left(J, H, \Lambda ; \bar{Q}^{\varkappa i}\right)$, we can now restate the above condition in the following way. The identity $u^{2} \bumpeq u$ is satisfied in $\mathcal{M}(J, H, \Lambda ; Q)$ if, for every $i \in J, \varkappa \in \Lambda$ and for every mapping $\vartheta: X \rightarrow J \times H \times \Lambda$ such that $\vartheta(z) \in\{i\} \times H \times\{\varkappa\}$, we have $\theta_{\varkappa i}\left(\zeta\left(u^{2}\right)\right)=\theta_{\varkappa i}(\zeta(u))$ where $\theta_{\varkappa i}: \mathcal{M}(X, G, X ; P) \rightarrow \mathcal{M}\left(J, H, \Lambda ; \bar{Q}^{\varkappa i}\right)$ is the homomorphism satisfying $\theta_{\varkappa i}(x, x, x)=\vartheta(x)$, for all $x \in X$.

Note that the equality $\theta_{\varkappa i}\left(\zeta\left(u^{2}\right)\right)=\theta_{\varkappa i}(\zeta(u))$ appearing in the condition stated at the end of the previous paragraph is the same as the equality $\theta_{\varkappa i}\left(\zeta(u)^{2}\right)=$ $\theta_{\varkappa i}(\zeta(u))$, and as $\mathrm{h}(u)=z=\mathrm{t}(u)$, in view of the former description of the homomorphism $\zeta: U(X) \rightarrow \mathcal{M}(X, G, X ; P)$ given in Result 2.1 , this equality is equivalent to the equality $\theta_{\varkappa i}\left((z, \mathrm{~m}(u), z)^{2}\right)=\theta_{\varkappa i}(z, \mathrm{~m}(u), z)$, and hence to the equality $\theta_{\varkappa i}\left(z, \mathrm{~m}(u)^{2}, z\right)=\theta_{\varkappa i}(z, \mathrm{~m}(u), z)$. 
Now, for every $i \in J, \varkappa \in \Lambda$ and for every mapping $\vartheta: X \rightarrow J \times H \times \Lambda$ such that $\vartheta(z) \in\{i\} \times H \times\{\varkappa\}$, one can check straightforwardly, on the basis of the definition of the homomorphism $\chi_{\varkappa i}^{\vartheta}: G \rightarrow H$ (see the definition of $\chi_{\varkappa i}^{\vartheta}$ before this proposition and the note after it), that the mapping

$$
\Upsilon_{\varkappa i}: \mathcal{M}(X, G, X ; P) \rightarrow \mathcal{M}\left(J, H, \Lambda ; \bar{Q}^{\varkappa i}\right)
$$

given by the formula

$$
(x, g, y) \mapsto\left(\varphi(x), \chi_{\varkappa i}^{\vartheta}(g), \psi(y)\right),
$$

for all $x, y \in X$ and $g \in G$, is a homomorphism of Rees matrix semigroups such that $\Upsilon_{\varkappa i}(x, x, x)=\vartheta(x)$, for all $x \in X$. However, the homomorphism $\theta_{\varkappa i}$ also satisfies $\theta_{\varkappa i}(x, x, x)=\vartheta(x)$, for all $x \in X$, and it is determined uniquely by this property. Consequently, this homomorphism $\theta_{\varkappa i}$ coincides with $\Upsilon_{\varkappa i}$, and hence it is completely described by the formula displayed above. Therefore, since $\varphi(z)=i$ and $\psi(z)=\varkappa$, for the given word $u \in U(X)$ with $\mathrm{h}(u)=z=\mathrm{t}(u)$, we have $\theta_{\varkappa i}\left(z, \mathrm{~m}(u)^{2}, z\right)=$ $\left(i, \chi_{\varkappa i}^{\vartheta}\left(\mathrm{m}(u)^{2}\right), \varkappa\right)=\left(i, \chi_{\varkappa i}^{\vartheta}(\mathrm{m}(u))^{2}, \varkappa\right)$ and $\theta_{\varkappa i}(z, \mathrm{~m}(u), z)=\left(i, \chi_{\varkappa i}^{\vartheta}(\mathrm{m}(u)), \varkappa\right)$. Thus the above equality $\theta_{\varkappa i}\left(z, \mathrm{~m}(u)^{2}, z\right)=\theta_{\varkappa i}(z, \mathrm{~m}(u), z)$ is equivalent to the equality $\chi_{\varkappa i}^{\vartheta}(\mathrm{m}(u))^{2}=\chi_{\varkappa i}^{\vartheta}(\mathrm{m}(u))$, that is, to the equality $\chi_{\varkappa i}^{\vartheta}(\mathrm{m}(u))=1$, as required.

We conclude this section by extracting from the previous proposition a criterion for the validity of nearly restricted identities of the form $v^{2} \bumpeq v$, for $v \in I U(X)$ with $\mathrm{h}(v)=z=\mathrm{t}(v)$, in arbitrary (idempotent generated) completely simple semigroups, that is, in an arbitrary Rees matrix semigroup, say $\mathcal{M}(J, H, \Lambda ; Q)$. For any elements $\varkappa \in \Lambda$ and $i \in J$ and for any mapping $\pi: X \rightarrow J \times \Lambda$ satisfying $\pi(z)=(i, \varkappa)$, consider the homomorphism

$$
\widehat{\chi}_{\varkappa i}^{\pi}: \widehat{G} \rightarrow H
$$

defined in the following way. Again, it is enough to determine it merely on the set $\left\{p_{x y}: x, y \in X-\{z\}\right\}$ of generators of $\widehat{G}$. First take the mappings $\mu: X \rightarrow J$ and $v: X \rightarrow \Lambda$ specified by the formula $\pi(x)=(\mu(x), v(x))$, for all $x \in X$. Then define the homomorphism $\widehat{\chi}_{\varkappa i}^{\pi}$ by means of the following assignments :

$$
p_{x y} \mapsto q_{\nu(x) i}^{-1} q_{\nu(x) \mu(y)} q_{\varkappa \mu(y)}^{-1} q_{\varkappa i}, \quad \text { for all } x, y \in X-\{z\} .
$$

As before, no harm will be done by including the same assignments also for $x=z$ or $y=z$, since these additional assignments will reduce to $1 \mapsto 1$.

Now we can state the promised criterion for the validity of the above nearly restricted identities in arbitrary Rees matrix semigroups:

Corollary 2.12. Let $\mathcal{M}(J, H, \Lambda ; Q)$ be a Rees matrix semigroup. Let $v \in I U(X)$ be any word such that $\mathrm{h}(v)=z=\mathrm{t}(v)$. Then the nearly restricted identity $v^{2} \bumpeq v$ is satisfied in $\mathcal{M}(J, H, \Lambda ; Q)$ if and only if, for every $i \in J, \varkappa \in \Lambda$ and for every mapping $\pi: X \rightarrow J \times \Lambda$ such that $\pi(z)=(i, \varkappa)$, the equality $\widehat{\chi}_{\varkappa i}^{\pi}(\mathrm{m}(v))=1$ holds in $H$.

Proof. One only has to notice that every mapping $\pi: X \rightarrow J \times \Lambda$ satisfying $\pi(z)=$ $(i, \varkappa)$ can be made up into a mapping $\vartheta: X \rightarrow J \times H \times \Lambda$ such that $\vartheta(z) \in\{i\} \times H \times$ $\{\varkappa\}$ which agrees with $\pi$ in the first and the last components of the image of every element of $X$. The homomorphism $\chi_{\varkappa i}^{\vartheta}: G \rightarrow H$ then restricts on the subgroup $\widehat{G}$ to 
the homomorphism $\widehat{\chi}_{\varkappa i}^{\pi}: \widehat{G} \rightarrow H$. Since all words $v \in I U(X)$ have the property that $\mathrm{m}(v) \in \widehat{G}$, it is enough to refer now to Proposition 2.11.

3. Some finite metabelian groups. Metabelian groups are solvable groups of derived length at most 2. Equivalently, metabelian groups are groups which are extensions of an abelian group by another abelian group. We provide now some instances of finite metabelian groups which will be useful in the subsequent sections of this paper. For every prime number $p$, we denote by $\mathcal{A}_{p}$ the variety of all abelian groups of exponent $p$. More specifically, we supply in this section certain finite groups from the Malcev product $\mathcal{A}_{p} \circ \mathcal{A}_{q}$, for distinct prime numbers $p, q$.

Thus let $p, q$ be two prime numbers such that $p \neq q$. Throughout this section, these prime numbers will be fixed. Let

$$
\langle a\rangle=\left\{1, a, a^{2}, \ldots, a^{p-1}\right\}
$$

be a cyclic group of order $p$ generated by an element $a$, and let

$$
\langle b\rangle=\left\{1, b, b^{2}, \ldots, b^{q-1}\right\}
$$

be a cyclic group of order $q$ generated by another element $b$. Let

$$
\langle a\rangle^{\langle b\rangle}=\{f:\langle b\rangle \rightarrow\langle a\rangle\}
$$

be the power of the group $\langle a\rangle$ indexed by elements of the group $\langle b\rangle$. Then the group $\langle b\rangle$ has a left action on the group $\langle a\rangle^{\langle b\rangle}$ given, for every $j \in\{0,1,2, \ldots, q-1\}$ and for every $\operatorname{map} f:\langle b\rangle \rightarrow\langle a\rangle$, by the formula

$$
b^{j} \cdot f={ }^{j} f
$$

where ${ }^{j} f:\langle b\rangle \rightarrow\langle a\rangle$ is the map defined, for every $k \in\{0,1,2, \ldots, q-1\}$, by the formula

$$
{ }^{j} f\left(b^{k}\right)=f\left(b^{j+k}\right)
$$

(the sum of exponents is taken modulo $q$, of course). For every $i \in\{0,1,2, \ldots, p-1\}$, let $c_{i}:\langle b\rangle \rightarrow\langle a\rangle$ be the constant map such that $c_{i}(\langle b\rangle)=\left\{a^{i}\right\}$. Consider the following subgroup of the group $\langle a\rangle^{\langle b\rangle}$ :

$$
\complement=\left\{c_{0}, c_{1}, c_{2}, \ldots, c_{p-1}\right\} .
$$

Since $\langle a\rangle^{\langle b\rangle}$ is an abelian group, $\complement$ is a normal subgroup in it, and so we may further consider the quotient group $\langle a\rangle^{\langle b\rangle} /$ C. Elements of this quotient group are the classes of maps $f$. $\complement$, for all maps $f:\langle b\rangle \rightarrow\langle a\rangle$. Notice that all constant maps $c_{0}, c_{1}, c_{2}, \ldots, c_{p-1}$ are fixed points of the above left action of $\langle b\rangle$ on the group $\langle a\rangle^{\langle b\rangle}$. Consequently, a left action of $\langle b\rangle$ on the quotient group $\langle a\rangle^{\langle b\rangle} / \mathcal{C}$ arises, which is given, for every $j \in\{0,1,2, \ldots, q-1\}$ and for every map $f:\langle b\rangle \rightarrow\langle a\rangle$, by the formula

$$
b^{j} \cdot(f \cdot \complement)={ }^{j} f \cdot \complement .
$$

We next show that this left action of $\langle b\rangle$ on $\langle a\rangle^{\langle b\rangle} / \complement$ no longer has any fixed point except the identity class $\complement$. More precisely, we check that every element of $\langle b\rangle$ except the identity 1 acting on $\langle a\rangle^{\langle b\rangle} / C$ in the way specified above permutes the classes of $\langle a\rangle^{\langle b\rangle} / \complement-\{\complement\}$ in such a way that this permutation has no fixed points. 
LEMma 3.1. For every $j \in\{1,2, \ldots, q-1\}$ and for every map $f:\langle b\rangle \rightarrow\langle a\rangle$ such that $f \notin \mathrm{C}$, we have

$$
b^{j} \cdot(f \cdot \complement) \neq f \cdot \complement .
$$

Proof. Assume, by contradiction, that for some $j \in\{1,2, \ldots, q-1\}$ and for some $\operatorname{map} f:\langle b\rangle \rightarrow\langle a\rangle, f \notin \complement$, we have $b^{j} \cdot(f \cdot \complement)=f \cdot \complement$. That is, we have ${ }^{j} f \cdot \complement=f \cdot \complement$, which means that ${ }^{j} f \cdot f^{-1} \in \complement$, so that, for some $i_{0} \in\{0,1,2, \ldots, p-1\}$, we have ${ }^{j} f \cdot f^{-1}=$ $c_{i_{0}}$ and hence ${ }^{j} f=c_{i_{0}} \cdot f$. By the definition of the map ${ }^{j} f$, this entails that $f\left(b^{j+k}\right)=$ $a^{i_{0}} \cdot f\left(b^{k}\right)$ holds for all $k \in\{0,1,2, \ldots, q-1\}$. Multiplying these equalities for all $k=$ $0,1,2, \ldots, q-1$, we obtain that

$$
f(1) \cdot f(b) \cdot f\left(b^{2}\right) \cdots \cdot f\left(b^{q-1}\right)=a^{q i_{0}} \cdot f(1) \cdot f(b) \cdot f\left(b^{2}\right) \cdots \cdot f\left(b^{q-1}\right),
$$

whence it follows that $a^{q i_{0}}=1$. Since the element $a$ is of order $p$ and $p, q$ are distinct prime numbers, this yields that $p \mid i_{0}$, so that $i_{0}=0$. Thus we get that ${ }^{j} f=c_{0} \cdot f$. However, since $c_{0}$ is the constant map such that $c_{0}(\langle b\rangle)=\{1\}$, this entails that ${ }^{j} f=f$. This means that $f\left(b^{j+k}\right)=f\left(b^{k}\right)$ holds for all $k \in\{0,1,2, \ldots, q-1\}$. In particular, this results in the equalities

$$
f(1)=f\left(b^{j}\right)=f\left(b^{2 j}\right)=\cdots=f\left(b^{(q-1) j}\right)
$$

(the exponents are again taken modulo $q$ ). As $j \in\{1,2, \ldots, q-1\}$ and $q$ is a prime number, the element $b^{j}$ is of order $q$, which means that $\langle b\rangle=\left\{1, b^{j}, b^{2 j}, \ldots, b^{(q-1) j}\right\}$. The equalities displayed above thus show that $f$ is a constant map, that is, $f \in \complement$, which is a contradiction.

Turning back to the left action of the group $\langle b\rangle$ on the quotient group $\langle a\rangle^{\langle b\rangle} / \complement$ defined above, we may use it to construct the semidirect product of the group $\langle a\rangle^{\langle b\rangle} / \complement$ by the group $\langle b\rangle$ determined by this left action. That is, we may consider the group

$$
K_{p, q}=\langle a\rangle^{\langle b\rangle} / \complement *\langle b\rangle
$$

whose elements are of the form $\left(f \cdot \complement, b^{j}\right)$, for all $j \in\{0,1,2, \ldots, q-1\}$ and for any maps $f:\langle b\rangle \rightarrow\langle a\rangle$, and the multiplication is given by the formula

$$
\left(f \cdot \complement, b^{j}\right) \cdot\left(g \cdot \complement, b^{k}\right)=\left(\left(f \cdot j_{g}\right) \cdot \complement, b^{j+k}\right),
$$

for all $j, k \in\{0,1,2, \ldots, q-1\}$ and for any maps $f, g:\langle b\rangle \rightarrow\langle a\rangle$ (the sum of exponents is again taken modulo $q$ ). Taking the identity 1 of the group $\langle b\rangle$, we may further consider the subgroup

$$
L_{p, q}=\langle a\rangle^{\langle b\rangle} / \complement *\{1\}
$$

of the group $K_{p, q}$ consisting of all elements of the form $(f \cdot \complement, 1)$, for arbitrary maps $f:\langle b\rangle \rightarrow\langle a\rangle$. Then $L_{p, q}$ is clearly a normal subgroup of $K_{p, q}$ and it is obviously isomorphic to the group $\langle a\rangle^{\langle b\rangle} / \complement$ which belongs to the variety $\mathcal{A}_{p}$. The quotient group $K_{p, q} / L_{p, q}$ is evidently isomorphic to the group $\langle b\rangle$ which belongs to the variety $\mathcal{A}_{q}$. Thus the group $K_{p, q}$ is an extension of the group $\langle a\rangle^{\langle b\rangle} / \complement$ from $\mathcal{A}_{p}$ by the group $\langle b\rangle$ from $\mathcal{A}_{q}$, and therefore the group $K_{p, q}$ itself belongs to the Malcev product $\mathcal{A}_{p} \circ \mathcal{A}_{q}$. The element $(\complement, 1)$ is the identity of $K_{p, q}$. 
LEMMA 3.2. All elements of the group $K_{p, q}$ occurring in the set $L_{p, q}-\{(\complement, 1)\}$ are of order $p$. All elements occurring in the set $K_{p, q}-L_{p, q}$ are of order $q$.

Proof. The first statement is obvious. As far as the second statement is concerned, every element in the set $K_{p, q}-L_{p, q}$ is of the form $\left(f \cdot \complement, b^{j}\right)$ for some $j \in\{1,2, \ldots, q-1\}$ and for some map $f:\langle b\rangle \rightarrow\langle a\rangle$. According to the above formula for multiplication in $K_{p, q}$, we obtain

$$
\underbrace{\left(f \cdot \complement, b^{j}\right) \cdots \cdots \cdot\left(f \cdot \complement, b^{j}\right)}_{q}=\left(f \cdot{ }^{j} f \cdot{ }^{2 j} f \cdots \cdots \cdot(q-1) j f \cdot \complement, b^{q j}\right)
$$

(indices at $f$ are computed modulo $q$, of course). However, since $q$ is a prime number and $j \in\{1,2, \ldots, q-1\}$, we have $\{0, j, 2 j, \ldots,(q-1) j\}=\{0,1,2, \ldots, q-1\}$ if the numbers in the first of these two sets are taken modulo $q$. Consequently, we see that $f \cdot{ }^{j} f \cdot{ }^{2 j} f \ldots .\left({ }^{q-1) j} f=f \cdot{ }^{1} f \cdot{ }^{2} f \ldots . \cdot{ }^{q-1} f\right.$. But $f \cdot{ }^{1} f \cdot{ }^{2} f \ldots . . q^{-1} f$ clearly is the constant map of $\langle b\rangle$ to the element $f(1) \cdot f(b) \cdot f\left(b^{2}\right) \cdots \cdot f\left(b^{q-1}\right)$ of $\langle a\rangle$, so that it belongs to $\complement$. Therefore, the product displayed above is equal to the identity $(\complement, 1)$ of $K_{p, q}$. This verifies that the elements $\left(f \cdot \complement, b^{j}\right)$ from the set $K_{p, q}-L_{p, q}$ are of order $q$.

LEMMA 3.3. For any pair of elements of the group $K_{p, q}$ such that one of them belongs to the set $L_{p, q}-\{(\complement, 1)\}$ and the other one belongs to the set $K_{p, q}-L_{p, q}$, it is the case that these two elements do not commute in $K_{p, q}$.

Proof. For any $j \in\{1,2, \ldots, q-1\}$ and for any maps $f, g:\langle b\rangle \rightarrow\langle a\rangle$ such that $f \notin \complement$, we obtain

$$
\begin{aligned}
& (f \cdot \complement, 1) \cdot\left(g \cdot \complement, b^{j}\right)=\left(f \cdot g \cdot \complement, b^{j}\right)=\left(g \cdot f \cdot \complement, b^{j}\right), \\
& \left(g \cdot \complement, b^{j}\right) \cdot(f \cdot \complement, 1)=\left(g \cdot{ }^{j} f \cdot \complement, b^{j}\right) .
\end{aligned}
$$

However, by Lemma 3.1 , we have $b^{j} \cdot(f \cdot \complement) \neq f \cdot \complement$, that is, ${ }^{j} f \cdot \complement \neq f \cdot \complement$, and hence $g \cdot{ }^{j} f \cdot \complement \neq g \cdot f \cdot \complement$. Consequently, we see that

$$
(f \cdot \complement, 1) \cdot\left(g \cdot \complement, b^{j}\right) \neq\left(g \cdot \complement, b^{j}\right) \cdot(f \cdot \complement, 1),
$$

as claimed.

LEMMA 3.4. For any pair of elements from the set $K_{p, q}-L_{p, q}$, it is true that these two elements commute in the group $K_{p, q}$ if and only if each of them is a positive power of the other one.

Proof. Clearly, it is enough to prove only the necessity of this condition. Thus assume that $j, k \in\{1,2, \ldots, q-1\}$ and that $f, g:\langle b\rangle \rightarrow\langle a\rangle$ are maps such that

$$
\left(f \cdot \complement, b^{j}\right) \cdot\left(g \cdot \complement, b^{k}\right)=\left(g \cdot \complement, b^{k}\right) \cdot\left(f \cdot \complement, b^{j}\right) .
$$

Let $\ell \in\{1,2, \ldots, q-1\}$ be that integer for which $j \cdot \ell \equiv k(\bmod q)$. Then from the previous equality we deduce that

$$
\left(f \cdot \complement, b^{j}\right)^{q-\ell} \cdot\left(g \cdot \complement, b^{k}\right)=\left(g \cdot \complement, b^{k}\right) \cdot\left(f \cdot \complement, b^{j}\right)^{q-\ell} .
$$


That is, putting $\hat{f}=f \cdot{ }^{j} f \cdot 2 j f \ldots .(q-\ell-1) j f$, we obtain that

$$
\left(\hat{f} \cdot{ }^{q-k} g \cdot \complement, 1\right)=\left(g \cdot{ }^{k} \hat{f} \cdot \complement, 1\right)
$$

so that $\hat{f} \cdot{ }^{q-k} g \cdot \complement=g \cdot{ }^{k} \hat{f} \cdot \complement$. Hence it follows that $b^{k} \cdot\left(g \cdot{ }^{k} \hat{f} \cdot \complement\right)=b^{k} \cdot\left(\hat{f} \cdot{ }^{q-k} g \cdot \complement\right)=$ ${ }^{k} \hat{f} \cdot g \cdot \complement=g \cdot k \hat{f} \cdot \complement$. By Lemma 3.1, this means that $g \cdot k \hat{f} \in \complement$. Therefore $g \cdot k \hat{f} \cdot \complement=\complement$, that is, $g \cdot \complement \cdot k \hat{f} \cdot \complement=\complement$. Thus $g \cdot \complement$ is the inverse of ${ }^{k} \hat{f} \cdot \complement$ in the group $\langle a\rangle^{\langle b\rangle} / \complement$. Now, putting $\bar{f}=f \cdot{ }^{j} f \cdot{ }^{2 j} f \ldots \ldots \cdot(\ell-1) j f$, we also obtain that

$$
\left(f \cdot \complement, b^{j}\right)^{q}=\left(f \cdot \complement, b^{j}\right)^{\ell} \cdot\left(f \cdot \complement, b^{j}\right)^{q-\ell}=\left(\bar{f} \cdot \complement, b^{k}\right) \cdot\left(\hat{f} \cdot \complement, b^{q-k}\right)=\left(\bar{f} \cdot{ }^{k} \hat{f} \cdot \complement, 1\right),
$$

while, by Lemma 3.2, we have

$$
\left(f \cdot \complement, b^{j}\right)^{q}=(\complement, 1) .
$$

This yields that $\bar{f} \cdot{ }^{k} \hat{f} \cdot \complement=\complement$, that is, $\bar{f} \cdot \complement \cdot{ }^{k} \hat{f} \cdot \complement=\complement$. Thus also $\bar{f} \cdot \complement$ is the inverse of ${ }^{k} \hat{f} \cdot \complement$ in the group $\langle a\rangle^{\langle b\rangle} / \complement$. Therefore we get that $g \cdot \complement=\bar{f} \cdot \complement$. Hence it follows that

$$
\left(g \cdot \complement, b^{k}\right)=\left(f \cdot \complement, b^{j}\right)^{\ell} .
$$

Letting $m \in\{1,2, \ldots, q-1\}$ be the integer for which $\ell \cdot m \equiv 1(\bmod q)$ and taking the $m$-th powers on both sides of this equality, we also obtain that

$$
\left(f \cdot \complement, b^{j}\right)=\left(g \cdot \complement, b^{k}\right)^{m},
$$

referring to Lemma 3.2 once again. This is what had to be shown.

4. Generating varieties of all completely simple semigroups having subgroups in prescribed varieties of groups. Given any variety $\mathcal{Q}$ of groups, we denote by $\mathcal{C}(\mathcal{Q})$ the variety of all completely simple semigroups whose maximal subgroups belong to $\mathcal{Q}$. In accordance with our notation introduced previously, $\mathcal{I C S}(\mathcal{Q})$ then stands for the near variety of all idempotent generated completely simple semigroups whose maximal subgroups belong to $\mathcal{Q}$.

We will show that, for any prime numbers $p, q$ such that $p \neq q$ and for any variety $\mathcal{V}$ of completely simple semigroups such that $\mathcal{C S}\left(\mathcal{A}_{p} \circ \mathcal{A}_{q}\right) \subseteq \mathcal{V}$, it is the case that the variety $\mathcal{V}$ is not generated by any finitely generated completely simple semigroup. This generalizes the well known fact that the variety $\mathcal{C S}$ of all completely simple semigroups itself is not generated by any finitely generated completely simple semigroup, see [8], VIII.10. As a matter of fact, we will see that none of the varieties $\mathcal{V}$ of completely simple semigroups satisfying $\mathcal{C S}\left(\mathcal{A}_{p} \circ \mathcal{A}_{q}\right) \subseteq \mathcal{V}$ is generated by a completely simple semigroup having either only finitely many $\mathcal{R}$-classes or only finitely many $\mathcal{L}$-classes. Our arguments will be of such a kind that they will document that the same statement holds true also for the near varieties $\mathcal{W}$ of idempotent generated completely simple semigroups satisfying $\mathcal{I C S}\left(\mathcal{A}_{p} \circ \mathcal{A}_{q}\right) \subseteq \mathcal{W}$.

Thus again let $p, q$ be any prime numbers such that $p \neq q$. We begin with a modification and simplification of some of the notation introduced in the previous section in connection with the group $K_{p, q}$ exhibited there. Recall that $K_{p, q}$ is the semidirect product of groups $\langle a\rangle^{\langle b\rangle} / \complement *\langle b\rangle$. As a first thing, we will denote by

$\mathfrak{s}$ - the identity $(\complement, 1)$ of the group $K_{p, q}$. 
Also the present notation for the set of all constant maps of $\langle b\rangle$ into $\langle a\rangle$, that is, the symbol $\complement$ for the identity class of the quotient group $\langle a\rangle^{\langle b\rangle} / \complement$ seems to be somewhat clumsy with regard to its further usage. Thus, from now on, we will denote by

$$
\text { II - the identity class } \complement \text { of }\langle a\rangle^{\langle b\rangle} / \complement \text {. }
$$

Furthermore, we will consider the following particular map $\partial:\langle b\rangle \rightarrow\langle a\rangle$ given by the formulas

$$
1 \mapsto a \quad \text { and } \quad b^{j} \mapsto 1, \quad \text { for all } j \in\{1,2, \ldots, q-1\} .
$$

Then we will denote by

$\Delta$ - the class $\partial \cdot \mathbb{I}$, that is, using the earlier notation, the class $\partial \cdot \complement$ of $\langle a\rangle^{\langle b\rangle} / \complement$.

In connection with the left action of $\langle b\rangle$ on $\langle a\rangle^{\langle b\rangle} / \complement$, that is, on $\langle a\rangle^{\langle b\rangle} / \mathbb{I I}$, which has been introduced in the previous section, we will use the following notation. For every $j \in\{0,1,2, \ldots, q-1\}$, we will denote the class $b^{j} \cdot \Delta$, that is, the class $b^{j} \cdot \partial \cdot \mathbb{I}={ }^{j} \partial \cdot \mathbb{I}$ simply by ${ }^{j} \Delta$. Instead of ${ }^{q-j} \Delta$ we will write only ${ }^{-j} \Delta$. Moreover, as is usual, $\Delta^{-1}$ will stand for the inverse of $\Delta$, that is, for the class $\Delta^{p-1}$ in $\langle a\rangle^{\langle b\rangle} / \mathbb{I I}$, and $b^{-1}$ will stand for the inverse of $b$, that is for the element $b^{q-1}$ in $\langle b\rangle$.

We next consider the elements

$$
(\mathbb{I}, b) \quad \text { and } \quad\left(\Delta, b^{-1}\right)
$$

of the group $K_{p, q}$. Both these elements belong to the set $K_{p, q}-L_{p, q}$ and the second one is certainly not a positive power of the first one. Therefore, by Lemma 3.4, these two elements do not commute in $K_{p, q}$. Moreover, it is a routine task to verify that, in fact, these two elements generate the entire group $K_{p, q}$.

Now we are ready to introduce the following Rees matrix semigroups which will have their role to play in this and the next sections of this paper. For every integer $n \geqslant 3$, we put $J_{n}=\{0,1,2, \ldots, n\}$ and we take the Rees matrix semigroup

$$
\mathcal{M}\left(J_{n}, K_{p, q}, J_{n} ; R_{n}\right)
$$

over the group $K_{p, q}$ whose sandwich matrix $R_{n}=\left({ }^{n} r_{i j}\right)_{i, j \in J_{n}}$ is described below. The entries of the matrix $R_{n}$ look as follows :

$$
\begin{aligned}
{ }^{n} r_{i i} & =(\mathbb{I}, b), \quad \text { for all } i \in\{1,2, \ldots, n\}, \\
{ }^{n} r_{i i+1} & =\left(\Delta, b^{-1}\right), \quad \text { for all } i \in\{1,2, \ldots, n-1\}, \\
{ }^{n} r_{n 1} & =\left(\Delta, b^{-1}\right), \quad \text { and } \\
{ }^{n} r_{i j} & =\Im \quad \text { otherwise. }
\end{aligned}
$$

Thus the sandwich matrix $R_{n}$ is normalized at the pair $(0,0)$ and each of its entries is equal either to the identity $\mathfrak{s}$ of $K_{p, q}$ or to one of the two elements $(\boldsymbol{I}, b),\left(\Delta, b^{-1}\right)$ of $K_{p, q}$ displayed above. Since, as we have noted, these two elements generate the whole group $K_{p, q}$, the Rees matrix semigroup $\mathcal{M}\left(J_{n}, K_{p, q}, J_{n} ; R_{n}\right)$ equals its core, see [8], III.2, and therefore it is idempotent generated. Thus, for every $n \geqslant 3$, the Rees matrix semigroup $\mathcal{M}\left(J_{n}, K_{p, q}, J_{n} ; R_{n}\right)$ belongs to the near variety $\mathcal{I C S}\left(\mathcal{A}_{p} \circ \mathcal{A}_{q}\right)$. For $n=5$ the sandwich matrix $R_{n}$ is shown in Figure 1. 


$$
\left(\begin{array}{cccccc}
\mathfrak{I} & \mathfrak{I} & \mathfrak{I} & \mathfrak{I} & \mathfrak{I} & \mathfrak{I} \\
\mathfrak{I} & (\mathbb{I I}, b) & \left(\Delta, b^{-1}\right) & \mathfrak{I} & \mathfrak{I} & \mathfrak{I} \\
\mathfrak{I} & \mathfrak{I} & (\mathbb{I}, b) & \left(\Delta, b^{-1}\right) & \mathfrak{I} & \mathfrak{I} \\
\mathfrak{I} & \mathfrak{I} & \mathfrak{I} & (\mathbb{I I}, b) & \left(\Delta, b^{-1}\right) & \mathfrak{I} \\
\mathfrak{I} & \mathfrak{I} & \mathfrak{I} & \mathfrak{I} & (\mathbb{I I}, b) & \left(\Delta, b^{-1}\right) \\
\mathfrak{I}\left(\Delta, b^{-1}\right) & \mathfrak{I} & \mathfrak{I} & \mathfrak{I} & (\mathbb{I I}, b)
\end{array}\right)
$$

Figure 1. The sandwich matrix $R_{5}$.

Remember that, given any group $H$, for any elements $g, h \in H$, the commutator $[g, h]$ of $g, h$ is defined to be the element of $H$ of the form

$$
[g, h]=g^{-1} h^{-1} g h .
$$

More generally, for any integer $n \geqslant 2$ and for any elements $h_{1}, h_{2}, \ldots, h_{n} \in H$, the $n$-fold commutator $\left[h_{1}, h_{2}, \ldots, h_{n}\right]$ is the element of $H$ defined by induction as follows. For $n=2$ it has just been done. If $n>2$ then the inductive definition proceeds by letting

$$
\left[h_{1}, h_{2}, \ldots, h_{n}\right]=\left[\left[h_{1}, h_{2}, \ldots, h_{n-1}\right], h_{n}\right]
$$

the formula relies on the above definition of the two-fold commutator.

The following observation regarding the group $K_{p, q}$ in connection with commutators will come in useful later.

ClAIM 4.1. For any integer $n \geqslant 1$ and for arbitrary elements $\mho_{0}, \mho_{1}, \mho_{2}, \ldots, \mho_{n}$ of the group $K_{p, q}$, we have

$$
\left[\mho_{0}, \mho_{1}, \mho_{2}, \ldots, \mho_{n}\right] \in L_{p, q} .
$$

Moreover, if $\mho_{0} \in L_{p, q}-\{\Im\}$ and $\mho_{1}, \mho_{2}, \ldots, \mho_{n} \in K_{p, q}-L_{p, q}$, then

$$
\left[\mho_{0}, \mho_{1}, \mho_{2}, \ldots, \mho_{n}\right] \neq \Im .
$$

On the other hand, if this inequality holds, then evidently $\mho_{0} \neq \mathfrak{s}$, and if, in addition, $\mho_{0} \in L_{p, q}-\{\Im\}$, then $\mho_{1}, \mho_{2}, \ldots, \mho_{n} \in K_{p, q}-L_{p, q}$.

Proof. The first statement is obvious, since the commutator of any two elements of the group $K_{p, q}$ belongs to the subgroup $L_{p, q}$. Assume that $\mho_{0} \in L_{p, q}-\{\Im\}$ and $\mho_{1}, \mho_{2}, \ldots, \mho_{n} \in K_{p, q}-L_{p, q}$. Then, using Lemma 3.3 , we can verify by induction that, for every $i \in\{1,2, \ldots, n\}$, we have $\left[\mho_{0}, \mho_{1}, \mho_{2}, \ldots, \mho_{i}\right] \in L_{p, q}-\{\Im\}$.

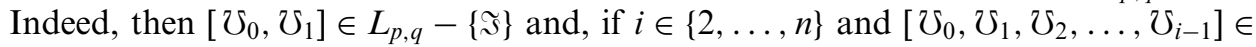

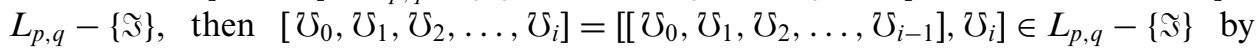
Lemma 3.3. Thus this assertion holds and, in particular, for $i=n$, we obtain the desired inequality. Assume further that $\mho_{0} \in L_{p, q}-\{\Im\}$. If $\mho_{1} \in L_{p, q}$, then $\left[\mho_{0}, \mho_{1}\right]=\mathfrak{s}$, and hence $\left[\mho_{0}, \mho_{1}, \mho_{2}, \ldots, \mho_{n}\right]=\mathfrak{s}$. If, for some $j \in\{2, \ldots, n\}$, we have $\mho_{j} \in L_{p, q}$, then, since $\left[\mho_{0}, \mho_{1}, \mho_{2}, \ldots, \mho_{j-1}\right] \in L_{p, q}$, we get $\left[\mho_{0}, \mho_{1}, \mho_{2}, \ldots, \mho_{j}\right]=$ $\left[\left[\mho_{0}, \mho_{1}, \mho_{2}, \ldots, \mho_{j-1}\right], \mho_{j}\right]=\mathfrak{s}$, and hence $\left[\mho_{0}, \mho_{1}, \mho_{2}, \ldots, \mho_{n}\right]=\mathfrak{\Im}$ again. These notes verify the last statement formulated above.

Again let $X$ be an infinite set containing a distinguished element $z \in X$. Remember the absolutely free group $G$ on the set $X \cup\left\{p_{x y}: x, y \in X-\{z\}\right\}$ introduced in Section 2. 
We next define, for every integer $n \geqslant 3$, an element $\pi_{n}$ of the group $G$ in the following way. We first choose pairwise distinct elements $x_{1}, x_{2}, \ldots, x_{n} \in X-\{z\}$. The element $\pi_{n}$ will then be defined in such a way that it will actually become an element of the absolutely free group on the set $\left\{p_{x_{i} x_{j}}: i, j \in\{1,2, \ldots, n\}\right\}$. The definition of the element $\pi_{n}$ will proceed by forming consecutively certain elements $\pi_{n}^{\mathrm{o}}, \pi_{n}^{11}, \pi_{n}^{12}, \pi_{n}^{22}, \pi_{n}^{23}, \ldots, \pi_{n}^{n-1 n}, \pi_{n}^{n n}, \pi_{n}^{n 1}$ of $G$ as follows. We first put

$$
\pi_{n}^{\mathrm{o}}=\left[p_{x_{1} x_{1}}, p_{x_{1} x_{2}}\right]
$$

and

$$
\pi_{n}^{11}=\left[\pi_{n}^{\mathrm{o}}, p_{x_{1} x_{1}} p_{x_{2} x_{1}}^{-1}, p_{x_{1} x_{1}} p_{x_{3} x_{1}}^{-1}, \ldots, p_{x_{1} x_{1}} p_{x_{n-1} x_{1}}^{-1}\right] .
$$

Then, for every $k=2, \ldots, n$, we put

$$
\pi_{n}^{k-1 k}=\left[\pi_{n}^{k-1 k-1}, p_{x_{k-1} x_{k}} p_{x_{k-1} x_{k+1}}^{-1}, p_{x_{k-1} x_{k}} p_{x_{k-1} x_{k+2}}^{-1}, \ldots, p_{x_{k-1} x_{k}} p_{x_{k-1} x_{n+k-2}}^{-1}\right]
$$

and

$$
\pi_{n}^{k k}=\left[\pi_{n}^{k-1 k}, p_{x_{k} x_{k}} p_{x_{k+1} x_{k}}^{-1}, p_{x_{k} x_{k}} p_{x_{k+2} x_{k}}^{-1}, \ldots, p_{x_{k} x_{k}} p_{x_{n+k-2} x_{k}}^{-1}\right] .
$$

Finally, we put

$$
\pi_{n}^{n 1}=\left[\pi_{n}^{n n}, p_{x_{n} x_{1}} p_{x_{n} x_{2}}^{-1}, p_{x_{n} x_{1}} p_{x_{n} x_{3}}^{-1}, \ldots, p_{x_{n} x_{1}} p_{x_{n} x_{n-1}}^{-1}\right] .
$$

Then we let

$$
\pi_{n}=\pi_{n}^{n 1}
$$

Note that all indices in these formulas are positive integers occurring within the interval $1,2, \ldots, n$, and that they are computed modulo $n$, if necessary.

Now remember that in section 2, in the text preceding Proposition 2.9, we have defined, for every element $g \in G-\{1\}$, the word $\gamma(g)$ of $U(X)$ having the properties that $\mathrm{h}(\gamma(g))=z=\mathrm{t}(\gamma(g))$ and $\mathrm{m}(\gamma(g))=g$. In addition, this definition of $\gamma(g)$ has been of such a form that, for every element $h \in \widehat{G}-\{1\}$, the word $\gamma(h)$ appears in $I U(X)$. In the previous paragraph, we have defined, for every integer $n \geqslant 3$, an element $\pi_{n}$ of the group $G$, distinct from the identity of $G$, which actually belongs to the subgroup $\widehat{G}$. Thus we can take, for every integer $n \geqslant 3$, the word

$$
u_{n}=\gamma\left(\pi_{n}\right)
$$

of $I U(X)$. Then we will work with the sequence of identities

$$
u_{n}^{2} \bumpeq u_{n} \quad \text { for all } n \geqslant 3 .
$$

Notice that it is, in fact, a sequence of nearly restricted identities. The following observation considers the validity of these identities in the Rees matrix semigroups introduced earlier in this section.

ClaIm 4.2. For every integer $n \geqslant 3$, the identity $u_{n}^{2} \bumpeq u_{n}$ is not satisfied in the Rees matrix semigroup $\mathcal{M}\left(J_{n}, K_{p, q}, J_{n} ; R_{n}\right)$.

Proof. Since, for every $n \geqslant 3, u_{n} \in I U(X)$ and $\mathrm{h}\left(u_{n}\right)=z=\mathrm{t}\left(u_{n}\right)$, according to Corollary 2.12 , in order to show that the nearly restricted identity $u_{n}^{2} \bumpeq u_{n}$ is not 
satisfied in $\mathcal{M}\left(J_{n}, K_{p, q}, J_{n} ; R_{n}\right)$, it suffices to find indices $k, \ell \in J_{n}$ and a mapping $\eta: X \rightarrow J_{n} \times J_{n}$ such that $\eta(z)=(k, \ell)$ and $\widehat{\chi}_{\ell k}^{\eta}\left(\mathrm{m}\left(u_{n}\right)\right) \neq \Im$ in $K_{p, q}$, where $\widehat{\chi}_{\ell k}^{\eta}: \widehat{G} \rightarrow$ $K_{p, q}$ is the homomorphism defined as in the text preceding Corollary 2.12 in section 2 . But $u_{n}=\gamma\left(\pi_{n}\right)$, and hence $\mathrm{m}\left(u_{n}\right)=\pi_{n}$. Therefore, given any integer $n \geqslant 3$, it is enough to find indices $k, \ell \in J_{n}$ and a mapping $\eta: X \rightarrow J_{n} \times J_{n}$ such that $\eta(z)=(k, \ell)$ and $\widehat{\chi}_{\ell k}^{\eta}\left(\pi_{n}\right) \neq \Im$.

Thus take $k=0, \ell=0$ and let $\eta: X \rightarrow J_{n} \times J_{n}$ be any mapping satisfying $\eta(z)=(0,0)$ and $\eta\left(x_{i}\right)=(i, i)$, for all $i \in\{1,2, \ldots, n\}$. Then, by the definition of the homomorphism $\widehat{\chi}_{00}^{\eta}: \widehat{G} \rightarrow K_{p, q}$, we have $\widehat{\chi}_{00}^{\eta}\left(p_{x_{i} x_{j}}\right)={ }^{n} r_{i j}$, for all $i, j \in\{1,2, \ldots, n\}$, where ${ }^{n} r_{i j}$ is the respective entry of the sandwich matrix $R_{n}$. Then we will see that $\widehat{\chi}_{00}^{\eta}\left(\pi_{n}\right) \in L_{p, q}-\{\mathfrak{I}\}$.

In order to verify this statement, it is essentially enough to unfold the formulas in the definition of the element $\pi_{n}$ of $\widehat{G}$, or rather the formulas stemming from them and determining the homomorphic image $\widehat{\chi}_{00}^{\eta}\left(\pi_{n}\right)$ of $\pi_{n}$ in $K_{p, q}$. First note that

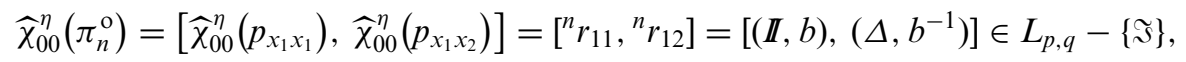

since this element, being a commutator of two elements of $K_{p, q}$, obviously belongs to $L_{p, q}$, but it is distinct from $\mathfrak{s}$, because the two elements $(\mathbb{I}, b),\left(\Delta, b^{-1}\right)$ do not commute in $K_{p, q}$, as we have seen above. Next observe that

$$
\begin{aligned}
\widehat{\chi}_{00}^{\eta}\left(\pi_{n}^{11}\right)= & {\left[\widehat{\chi}_{00}^{\eta}\left(\pi_{n}^{o}\right), \widehat{\chi}_{00}^{\eta}\left(p_{x_{1} x_{1}}\right) \widehat{\chi}_{00}^{\eta}\left(p_{x_{2} x_{1}}\right)^{-1}, \widehat{\chi}_{00}^{\eta}\left(p_{x_{1} x_{1}}\right) \widehat{\chi}_{00}^{\eta}\left(p_{x_{3} x_{1}}\right)^{-1}, \ldots\right.} \\
& \left.\ldots, \widehat{\chi}_{00}^{\eta}\left(p_{x_{1} x_{1}}\right) \widehat{\chi}_{00}^{\eta}\left(p_{x_{n-1} x_{1}}\right)^{-1}\right] \\
& =\left[\widehat{\chi}_{00}^{\eta}\left(\pi_{n}^{o}\right),{ }^{n} r_{11}{ }^{n} r_{21}^{-1},{ }^{n} r_{11}{ }^{n} r_{31}^{-1}, \ldots,{ }^{n} r_{11}{ }^{n} r_{n-11}^{-1}\right] \\
& =\left[\widehat{\chi}_{00}^{\eta}\left(\pi_{n}^{o}\right),(\boldsymbol{I}, b),(\mathbb{I}, b), \ldots,(\boldsymbol{I}, b)\right] \in L_{p, q}-\{\Im\},
\end{aligned}
$$

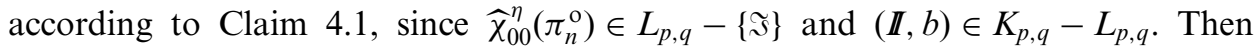
proceed further by observing that

$$
\begin{aligned}
\widehat{\chi}_{00}^{\eta}\left(\pi_{n}^{12}\right)= & {\left[\widehat{\chi}_{00}^{\eta}\left(\pi_{n}^{11}\right), \widehat{\chi}_{00}^{\eta}\left(p_{x_{1} x_{2}}\right) \widehat{\chi}_{00}^{\eta}\left(p_{x_{1} x_{3}}\right)^{-1}, \widehat{\chi}_{00}^{\eta}\left(p_{x_{1} x_{2}}\right) \widehat{\chi}_{00}^{\eta}\left(p_{x_{1} x_{4}}\right)^{-1}, \ldots\right.} \\
& \left.\ldots, \widehat{\chi}_{00}^{\eta}\left(p_{x_{1} x_{2}}\right) \widehat{\chi}_{00}^{\eta}\left(p_{x_{1} x_{n}}\right)^{-1}\right] \\
= & {\left[\widehat{\chi}_{00}^{\eta}\left(\pi_{n}^{11}\right),{ }^{n} r_{12}{ }^{n} r_{13}^{-1},{ }^{n} r_{12}{ }^{n} r_{14}^{-1}, \ldots,{ }^{n} r_{12}{ }^{n} r_{1 n}^{-1}\right] } \\
= & {\left[\widehat{\chi}_{00}^{\eta}\left(\pi_{n}^{11}\right),\left(\Delta, b^{-1}\right),\left(\Delta, b^{-1}\right), \ldots,\left(\Delta, b^{-1}\right)\right] \in L_{p, q}-\{\Im\}, }
\end{aligned}
$$

again by Claim 4.1, since $\widehat{\chi}_{00}^{\eta}\left(\pi_{n}^{11}\right) \in L_{p, q}-\{\mathfrak{I}\}$ and $\left(\Delta, b^{-1}\right) \in K_{p, q}-L_{p, q}$. In this manner, we can continue inspecting the $(n-1)$-fold commutators defining consecutively the further elements $\widehat{\chi}_{00}^{\eta}\left(\pi_{n}^{22}\right), \widehat{\chi}_{00}^{\eta}\left(\pi_{n}^{23}\right), \ldots, \widehat{\chi}_{00}^{\eta}\left(\pi_{n}^{n-1 n}\right), \widehat{\chi}_{00}^{\eta}\left(\pi_{n}^{n n}\right)$, until we reach the element $\hat{\chi}_{00}^{\eta}\left(\pi_{n}^{n 1}\right)=\widehat{\chi}_{00}^{\eta}\left(\pi_{n}\right)$. In this way, we arrive at the conclusion that

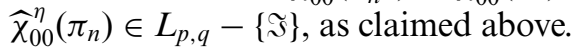

Now we are ready to prove the result announced in the preface to this section.

Proposition 4.3. No variety $\mathcal{V}$ of completely simple semigroups having the property that $\mathcal{C S}\left(\mathcal{A}_{p} \circ \mathcal{A}_{q}\right) \subseteq \mathcal{V}$, where $p$, q are distinct prime numbers, is generated by a completely simple semigroup having only finitely many $\mathcal{R}$-classes or by a completely simple semigroup having only finitely many $\mathcal{L}$-classes.

Proof. By contradiction, suppose that some variety $\mathcal{V}$ satisfying $\mathcal{C S}\left(\mathcal{A}_{p} \circ \mathcal{A}_{q}\right) \subseteq \mathcal{V}$ with $p, q$ as above is generated by a completely simple semigroup $S$ having finitely many 
$\mathcal{R}$-classes, say. Let $\mathcal{M}(I, H, \Lambda ; Q)$ be a Rees matrix representation of this semigroup $S$. Then the set $I$ is finite. Let $I$ have $m$ elements. Then $m \geqslant 2$, as $\mathcal{R B} \subseteq \mathcal{V}$. We shall show subsequently that then the semigroup $\mathcal{M}(I, H, \Lambda ; Q)$ satisfies the identities $u_{n}^{2} \bumpeq u_{n}$ for all integers $n>m$. Since this semigroup generates $\mathcal{V}$, the variety $\mathcal{V}$ should satisfy these identities, as well. However, since $\mathcal{C S}\left(\mathcal{A}_{p} \circ \mathcal{A}_{q}\right) \subseteq \mathcal{V}$, the variety $\mathcal{V}$ contains the Rees matrix semigroups $\mathcal{M}\left(J_{n}, K_{p, q}, J_{n} ; R_{n}\right)$ introduced earlier in this section, for all $n \geqslant 3$. But this means, according to Claim 4.2, that none of the identities $u_{n}^{2} \bumpeq u_{n}$ for $n \geqslant 3$ are satisfied in $\mathcal{V}$, which will be the desired contradiction.

Thus take any integer $n>m$ and consider the corresponding identity $u_{n}^{2} \bumpeq u_{n}$. Since $u_{n} \in I U(X)$ and $\mathrm{h}\left(u_{n}\right)=z=\mathrm{t}\left(u_{n}\right)$, according to Corollary 2.12, in order to show that the identity $u_{n}^{2} \bumpeq u_{n}$ is satisfied in the semigroup $\mathcal{M}(I, H, \Lambda ; Q)$, we need to verify that, for every $j \in I, \varkappa \in \Lambda$ and for every mapping $\eta: X \rightarrow I \times \Lambda$ such that $\eta(z)=(j, \varkappa)$, we have $\widehat{\chi}_{\varkappa j}^{\eta}\left(\mathrm{m}\left(u_{n}\right)\right)=1$ in $H$ where $\widehat{\chi}_{\varkappa j}^{\eta}: \widehat{G} \rightarrow H$ is the homomorphism defined in section 2 in the text preceding Corollary 2.12. Since $u_{n}=\gamma\left(\pi_{n}\right)$, we have $\mathrm{m}\left(u_{n}\right)=\pi_{n}$ in this condition. Thus we need to check that, for every $j \in I, \varkappa \in \Lambda$ and for every mapping $\eta: X \rightarrow$ $I \times \Lambda$ such that $\eta(z)=(j, \varkappa)$, we have $\widehat{\chi}_{\varkappa j}^{\eta}\left(\pi_{n}\right)=1$. Let, in this situation, $\mu: X \rightarrow I$ and $v: X \rightarrow \Lambda$ be the mappings determined by the formula $\eta(x)=(\mu(x), v(x))$, for all $x \in X$. Further, let the sandwich matrix $Q$ be of the form $Q=\left(q_{\lambda i}\right)_{\lambda \in \Lambda, i \in I}$. Remember from section 2 that then the homomorphism $\widehat{\chi}_{\varkappa j}^{\eta}: \widehat{G} \rightarrow H$ is defined in such a way that, for every $k, \ell \in\{1,2, \ldots, n\}$, we have $\hat{\chi}_{\varkappa j}^{\eta}\left(p_{x_{k} x_{\ell}}\right)=q_{v\left(x_{k}\right) j}^{-1} q_{v\left(x_{k}\right) \mu\left(x_{\ell}\right)} q_{\varkappa \mu\left(x_{\ell}\right)}^{-1} q_{\varkappa j}$. Now since the set $I$ has only $m$ elements and $n>m$, there exist indices $\imath, \varepsilon \in\{1,2, \ldots, n\}$ satisfying $\imath<\varepsilon$ such that $\mu\left(x_{\imath}\right)=\mu\left(x_{\varepsilon}\right)$. From the just mentioned characteristics of the homomorphism $\hat{\chi}_{\varkappa j}^{\eta}$ it becomes evident that the equality $\mu\left(x_{\imath}\right)=\mu\left(x_{\varepsilon}\right)$ entails that, for every $k \in\{1,2, \ldots, n\}$, we have $\widehat{\chi}_{\varkappa j}^{\eta}\left(p_{x_{k} x_{l}}\right)=\widehat{\chi}_{\varkappa j}^{\eta}\left(p_{x_{k} x_{\varepsilon}}\right)$. Looking at the definition of the elements $\pi_{n}^{11}, \pi_{n}^{12}, \pi_{n}^{22}, \pi_{n}^{23}, \ldots, \pi_{n}^{n-1 n}, \pi_{n}^{n n}, \pi_{n}^{n 1}$, we observe that if $1<\imath<\varepsilon$ then $p_{x_{l-1} x_{l}} p_{x_{l-1} x_{\varepsilon}}^{-1}$ appears among the elements in the $(n-1)$-fold commutator defining the element $\pi_{n}^{\imath-1 \imath}$, if $\imath+1<\varepsilon$ then $p_{x_{\varepsilon-1} x_{\varepsilon}} p_{x_{\varepsilon-1} x_{\imath}}^{-1}$ appears among the elements in the $(n-1)$-fold commutator defining the element $\pi_{n}^{\varepsilon-1 \varepsilon}$, and if $\imath=1$ and $\varepsilon=2$ then we note that $p_{x_{n} x_{1}} p_{x_{n} x_{2}}^{-1}$ appears among the elements in the $(n-1)$-fold commutator defining the element $\pi_{n}^{n 1}$. Hence it turns out that if $1<\imath<\varepsilon$ then $\widehat{\chi}_{2 j}^{\eta}\left(p_{x_{l-1} x_{\imath}}\right) \widehat{\chi}_{\varkappa j}^{\eta}\left(p_{x_{l-1} x_{\varepsilon}}\right)^{-1}=1$ and so $\hat{\chi}_{\varkappa j}^{\eta}\left(\pi_{n}^{\imath-1 \imath}\right)=1$, if $\imath+1<\varepsilon$ then $\widehat{\chi}_{\varkappa j}^{\eta}\left(p_{x_{\varepsilon-1} x_{\varepsilon}}\right) \widehat{\chi}_{\varkappa j}^{\eta}\left(p_{x_{\varepsilon-1} x_{l}}\right)^{-1}=1$ and so $\widehat{\chi}_{\varkappa j}^{\eta}\left(\pi_{n}^{\varepsilon-1 \varepsilon}\right)=$ 1 , and if $\iota=1$ and $\varepsilon=2$ then $\widehat{\chi}_{\varkappa j}^{\eta}\left(p_{x_{n} x_{1}}\right) \widehat{\chi}_{\varkappa j}^{\eta}\left(p_{x_{n} x_{2}}\right)^{-1}=1$ and so $\widehat{\chi}_{\varkappa j}^{\eta}\left(\pi_{n}^{n 1}\right)=1$. In every case, we thus eventually come to the conclusion that $\hat{\chi}_{\varkappa j}^{\eta}\left(\pi_{n}\right)=1$, which we needed to verify.

Looking through the arguments used in the proof of Proposition 4.3, we readily ascertain that, in parallel with the statement formulated in this proposition concerning varieties of completely simple semigroups, we have proved also the analogous statement concerning near varieties of idempotent generated completely simple semigroups, which has also been mentioned in the opening paragraphs of this section. Namely, we have also verified that no near variety $\mathcal{W}$ of idempotent generated completely simple semigroups having the property that $\mathcal{I C S}\left(\mathcal{A}_{p} \circ \mathcal{A}_{q}\right) \subseteq \mathcal{W}$, where $p, q$ are distinct prime numbers, is generated by a semigroup having only finitely many $\mathcal{R}$-classes or by a semigroup having only finitely many $\mathcal{L}$-classes.

5. Uncountably many near varieties of idempotent generated completely simple semigroups with metabelian subgroups. In this final section, we intend to prove the following result, which has been the real motivation for writing this paper. Remember 
once more that, for any prime numbers $p, q$ such that $p \neq q, \mathcal{I C S}\left(\mathcal{A}_{p} \circ \mathcal{A}_{q}\right)$ stands for the near variety of all idempotent generated completely simple semigroups whose maximal subgroups belong to the Malcev product $\mathcal{A}_{p} \circ \mathcal{A}_{q}$, which group variety consists, of course, of metabelian groups.

THEOREM 5.1. For arbitrary prime numbers $p, q$ such that $p \neq q$, there exist $2 \$^{\$_{0}}$ near varieties of idempotent generated completely simple semigroups occurring in the interval between the near variety $\mathcal{R B}$ of all rectangular bands and the near variety $\mathcal{I C S}\left(\mathcal{A}_{p} \circ \mathcal{A}_{q}\right)$ mentioned above.

In view of Theorem 1.4 or, more specifically, in view of Proposition 2.10, Theorem 5.1 will emerge as an immediate consequence of Proposition 5.2 which is stated below. In this proposition, certain nearly restricted identities will appear which we must first introduce.

Thus let again $X$ be an infinite set containing a distinguished element $z \in X$. Remember once more the absolutely free group $G$ on the set $X \cup\left\{p_{x y}: x, y \in X-\{z\}\right\}$ introduced in section 2. Now, for every integer $n \geqslant 7$, we define an element $\omega_{n}$ of the group $G$ in the following manner. As in the previous section, we first choose pairwise distinct elements $x_{1}, x_{2}, \ldots, x_{n} \in X-\{z\}$. Then the element $\omega_{n}$ will be defined in such a way that, in fact, it will belong to the absolutely free group on the set $\left\{p_{x_{i} x_{j}}: i, j \in\{1,2, \ldots, n\}\right\}$. This element $\omega_{n}$ will arise as the last element of the sequence of elements $\omega_{n}^{\mathrm{o}}, \omega_{n}^{11}, \omega_{n}^{12}, \omega_{n}^{22}, \omega_{n}^{23}, \ldots, \omega_{n}^{n-1 n}, \omega_{n}^{n n}, \omega_{n}^{n 1}$ of $G$ which are consecutively formed as follows. We begin by putting

$$
\omega_{n}^{\mathrm{o}}=\left[p_{x_{1} x_{1}}, p_{x_{1} x_{2}}\right]
$$

and

$$
\begin{gathered}
\omega_{n}^{11}=\left[\omega_{n}^{\mathrm{o}}, p_{x_{1} x_{1}}, p_{x_{1} x_{1}} p_{x_{2} x_{1}}^{-1}, p_{x_{1} x_{1}} p_{x_{3} x_{1}}^{-1}, p_{x_{1} x_{1}} p_{x_{4} x_{1}}^{-1}, p_{x_{1} x_{1}} p_{x_{5} x_{1}}^{-1}, p_{x_{1} x_{1}} p_{x_{6} x_{1}}^{-1},\right. \\
p_{x_{1} x_{1}} p_{x_{1} x_{n}}^{-1}, p_{x_{1} x_{1}} p_{x_{1} x_{n-1}}^{-1}, p_{x_{1} x_{1}} p_{x_{1} x_{n-2}}^{-1}, p_{x_{1} x_{1}} p_{x_{1} x_{n-3}}^{-1}, p_{x_{1} x_{1}} p_{x_{1} x_{n-4}}^{-1}, \\
\left.p_{x_{1} x_{1}} p_{x_{n} x_{1}}^{2}, p_{x_{1} x_{1}} p_{x_{1} x_{2}}^{2}\right] .
\end{gathered}
$$

Then we continue, for every $k=2, \ldots, n$, by putting

$$
\begin{gathered}
\omega_{n}^{k-1 k}=\left[\omega_{n}^{k-1 k-1}, p_{x_{k-1} x_{k}}, p_{x_{k-1} x_{k}} p_{x_{k-1} x_{k+1}}^{-1}, p_{x_{k-1} x_{k}} p_{x_{k-1} x_{k+2}}^{-1},\right. \\
p_{x_{k-1} x_{k}} p_{x_{k-1} x_{k+3}}^{-1}, p_{x_{k-1} x_{k}} p_{x_{k-1} x_{k+4}}^{-1}, p_{x_{k-1} x_{k}} p_{x_{k-1} x_{k+5}}^{-1}, \\
p_{x_{k-1} x_{k}} p_{x_{k-2} x_{k}}^{-1}, p_{x_{k-1} x_{k}} p_{x_{k-3} x_{k}}^{-1}, p_{x_{k-1} x_{k}} p_{x_{k-4} x_{k}}^{-1}, \\
p_{x_{k-1} x_{k}} p_{x_{k-5} x_{k}}^{-1}, p_{x_{k-1} x_{k}} p_{x_{k-6} x_{k}}^{-1}, \\
\left.p_{x_{k-1} x_{k}} p_{x_{k-1} x_{k-1}}^{2}, p_{x_{k-1} x_{k}} p_{x_{k} x_{k}}^{2}\right]
\end{gathered}
$$

and

$$
\begin{gathered}
\omega_{n}^{k k}=\left[\omega_{n}^{k-1 k}, p_{x_{k} x_{k}}, p_{x_{k} x_{k}} p_{x_{k+1} x_{k}}^{-1}, p_{x_{k} x_{k}} p_{x_{k+2} x_{k}}^{-1}, p_{x_{k} x_{k}} p_{x_{k+3} x_{k}}^{-1},\right. \\
p_{x_{k} x_{k}} p_{x_{k+4} x_{k}}^{-1}, p_{x_{k} x_{k}} p_{x_{k+5} x_{k}}^{-1}, \\
p_{x_{k} x_{k}} p_{x_{k} x_{k-1}}^{-1}, p_{x_{k} x_{k}} p_{x_{k} x_{k-2}}^{-1}, p_{x_{k} x_{k}} p_{x_{k} x_{k-3}}^{-1}, \\
p_{x_{k} x_{k}} p_{x_{k} x_{k-4}}^{-1}, p_{x_{k} x_{k}} p_{x_{k} x_{k-5}}^{-1}, \\
\left.p_{x_{k} x_{k}} p_{x_{k-1} x_{k}}^{2}, p_{x_{k} x_{k}} p_{x_{k} x_{k+1}}^{2}\right] .
\end{gathered}
$$


Finally, we end by putting

$$
\begin{aligned}
\omega_{n}^{n 1}=\left[\omega_{n}^{n n}, p_{x_{n} x_{1}}, p_{x_{n} x_{1}} p_{x_{n} x_{2}}^{-1}, p_{x_{n} x_{1}} p_{x_{n} x_{3}}^{-1}, p_{x_{n} x_{1}} p_{x_{n} x_{4}}^{-1}, p_{x_{n} x_{1}} p_{x_{n} x_{5}}^{-1}, p_{x_{n} x_{1}} p_{x_{n} x_{6}}^{-1},\right. \\
\quad p_{x_{n} x_{1}} p_{x_{n-1} x_{1}}^{-1}, p_{x_{n} x_{1}} p_{x_{n-2} x_{1}}^{-1}, p_{x_{n} x_{1}} p_{x_{n-3} x_{1}}^{-1}, p_{x_{n} x_{1}} p_{x_{n-4} x_{1}}^{-1}, p_{x_{n} x_{1}} p_{x_{n-5} x_{1}}^{-1}, \\
\left.\quad p_{x_{n} x_{1}} p_{x_{n} x_{n}}^{2}, p_{x_{n} x_{1} x_{1}} p_{x_{1} x_{1}}^{2}\right] .
\end{aligned}
$$

Having this done, we let

$$
\omega_{n}=\omega_{n}^{n 1}
$$

We again point out that all indices appearing in these formulas are positive integers occurring within the interval $1,2, \ldots, n$, and that they are computed modulo $n$, if necessary. For instance, in the 14-fold commutator defining the element $\omega_{n}^{k k}$ for $k=n$, the element appearing last is $p_{x_{n} x_{n}} p_{x_{n} x_{1}}^{2}$.

Next remember once again that in section 2, in the text preceding Proposition 2.9, we have defined, for every element $g \in G-\{1\}$, the word $\gamma(g)$ of $U(X)$ having the properties that $\mathrm{h}(\gamma(g))=z=\mathrm{t}(\gamma(g))$ and $\mathrm{m}(\gamma(g))=g$. Moreover, this definition of $\gamma(g)$ has been of such a form that, for every element $h \in \widehat{G}-\{1\}$, the word $\gamma(h)$ belongs to $I U(X)$. Now, in the previous paragraph, we have defined, for every integer $n \geqslant 7$, an element $\omega_{n}$ of the group $G$, distinct from the identity of $G$, which actually occurs in the subgroup $\widehat{G}$. Thus, for every integer $n \geqslant 7$, we have in hand the word

$$
w_{n}=\gamma\left(\omega_{n}\right)
$$

of $I U(X)$. We will further use the sequence of identities

$$
w_{n}^{2} \bumpeq w_{n} \quad \text { for all integers } n \geqslant 7 .
$$

Subsequently, however, we will rather deal with the selected subsequence of identities

$$
w_{s}^{2} \bumpeq w_{s} \quad \text { for all prime numbers } s \geqslant 7 .
$$

Notice again that all these identities are, in fact, nearly restricted identities.

Now we can state the promised proposition which yields the above Theorem 5.1 as a direct consequence.

Proposition 5.2. For any prime numbers $p, q$ such that $p \neq q$, it is the case that the identities $w_{s}^{2} \bumpeq w_{s}$, for all prime numbers $s \geqslant 7$, form an infinite independent set of nearly restricted identities within the near variety $\mathcal{I C S}\left(\mathcal{A}_{p} \circ \mathcal{A}_{q}\right)$. That is, none of these identities is a consequence of the other ones within $\operatorname{ICS}\left(\mathcal{A}_{p} \circ \mathcal{A}_{q}\right)$.

This proposition will ensue from the next two statements which both have to do with the Rees matrix semigroups $\mathcal{M}\left(J_{n}, K_{p, q}, J_{n} ; R_{n}\right)$ over the group $K_{p, q}$ introduced in the previous section. Remember in this context that, for all integers $n \geqslant 3$, these Rees matrix semigroups $\mathcal{M}\left(J_{n}, K_{p, q}, J_{n} ; R_{n}\right)$ are members of the near variety $\mathcal{I C S}\left(\mathcal{A}_{p} \circ \mathcal{A}_{q}\right)$.

Proposition 5.2 means that distinct subsets of the family of nearly restricted identities $w_{s}^{2} \bumpeq w_{s}$ where $s \geqslant 7$ are arbitrary prime numbers, when considered within the near variety $\mathcal{I C S}\left(\mathcal{A}_{p} \circ \mathcal{A}_{q}\right)$, determine distinct near subvarieties of this near variety. In addition, all near subvarieties arising in this manner contain the class $\mathcal{R B}$ of all rectangular bands. Thus, in this way, we obtain $2^{\aleph_{0}}$ near subvarieties of $\mathcal{I C S}\left(\mathcal{A}_{p} \circ \mathcal{A}_{q}\right)$ containing all rectangular bands. 
ClaIM 5.3. For every integer $n \geqslant 7$, the identity $w_{n}^{2} \bumpeq w_{n}$ is not satisfied in the Rees matrix semigroup $\mathcal{M}\left(J_{n}, K_{p, q}, J_{n} ; R_{n}\right)$.

Proof. We proceed entirely analogously to the proof of Claim 4.2 in the previous section. Referring to Corollary 2.12 again and arguing in the same way as before, we come to the following criterion. Given any integer $n \geqslant 7$, in order to establish that the nearly restricted identity $w_{n}^{2} \bumpeq w_{n}$ is not satisfied in $\mathcal{M}\left(J_{n}, K_{p, q}, J_{n} ; R_{n}\right)$, it suffices to find indices $k, \ell \in J_{n}$ and a mapping $\pi: X \rightarrow J_{n} \times J_{n}$ such that $\pi(z)=(k, \ell)$ and $\widehat{\chi}_{\ell k}^{\pi}\left(\omega_{n}\right) \neq \mathfrak{s}$, where $\widehat{\chi}_{\ell k}^{\pi}: \widehat{G} \rightarrow K_{p, q}$ is the homomorphism defined as in the text preceding Corollary 2.12 in section 2.

Thus, as in the proof of Claim 4.2, take $k=0, \ell=0$ and let $\pi: X \rightarrow J_{n} \times J_{n}$ be any mapping satisfying $\pi(z)=(0,0)$ and $\pi\left(x_{i}\right)=(i, i)$, for all $i \in\{1,2, \ldots, n\}$. Then, according to the definition of $\widehat{\chi}_{00}^{\pi}: \widehat{G} \rightarrow K_{p, q}$, we have $\widehat{\chi}_{00}^{\pi}\left(p_{x_{i} x_{j}}\right)={ }^{n} r_{i j}$, for all $i, j \in$ $\{1,2, \ldots, n\}$, where ${ }^{n} r_{i j}$ is the respective entry of the sandwich matrix $R_{n}$. Then, similarly as before, we will see that $\widehat{\chi}_{00}^{\pi}\left(\omega_{n}\right) \in L_{p, q}-\{\mathfrak{I}\}$.

In order to verify this statement, it is again essentially enough to unfold the formulas according to which the element $\widehat{\chi}_{00}^{\pi}\left(\omega_{n}\right)$ of $K_{p, q}$ is consecutively calculated. Notice that these formulas stem from those appearing in the above definition of the element $\omega_{n}$ of $\widehat{G}$. Thus, we first note that

$$
\widehat{\chi}_{00}^{\pi}\left(\omega_{n}^{o}\right)=\left[\widehat{\chi}_{00}^{\pi}\left(p_{x_{1} x_{1}}\right), \widehat{\chi}_{00}^{\pi}\left(p_{x_{1} x_{2}}\right)\right]=\left[{ }^{n} r_{11},{ }^{n} r_{12}\right]=\left[(\mathbb{I}, b),\left(\Delta, b^{-1}\right)\right] \in L_{p, q}-\{\Im\},
$$

just as in the proof of Claim 4.2. Next, examining similarly the formula defining the element $\omega_{n}^{11}$ of $\widehat{G}$ and determining thus its homomorphic image $\widehat{\chi}_{00}^{\pi}\left(\omega_{n}^{11}\right)$ in $K_{p, q}$, we observe that

$$
\begin{aligned}
\widehat{\chi}_{00}^{\pi}\left(\omega_{n}^{11}\right)= & {\left[\widehat{\chi}_{00}^{\pi}\left(\omega_{n}^{\mathrm{o}}\right),{ }^{n} r_{11},{ }^{n} r_{11}{ }^{n} r_{21}^{-1},{ }^{n} r_{11}{ }^{n} r_{31}^{-1},{ }^{n} r_{11}{ }^{n} r_{41}^{-1},{ }^{n} r_{11}{ }^{n} r_{51}^{-1},{ }^{n} r_{11}{ }^{n} r_{61}^{-1},\right.} \\
& \left.{ }^{n} r_{11}{ }^{n} r_{1 n}^{-1},{ }^{n} r_{11}{ }^{n} r_{1 n-1}^{-1},{ }^{n} r_{11}{ }^{n} r_{1 n-2}^{-1},{ }^{n} r_{11}{ }^{n} r_{1 n-3}^{-1},{ }^{n} r_{11}{ }^{n} r_{1 n-4}^{-1},{ }^{n} r_{11}{ }^{n} r_{n 1}^{2},{ }^{n} r_{11}{ }^{n} r_{12}^{2}\right] \\
= & {[\widehat{\chi}_{00}^{\pi}\left(\omega_{n}^{\mathrm{o}}\right),(\mathbb{I}, b), \underbrace{(\mathbb{I}, b), \ldots,(\mathbb{I}, b)}_{10},\left({ }^{1} \Delta \cdot \Delta, b^{-1}\right),\left({ }^{1} \Delta \cdot \Delta, b^{-1}\right)] \in L_{p, q}-\{\Im\}, }
\end{aligned}
$$

in view of Claim 4.1, since $\widehat{\chi}_{00}^{\pi}\left(\omega_{n}^{o}\right) \in L_{p, q}-\{\Im\}$ and $(\mathbb{I}, b),\left({ }^{1} \Delta \cdot \Delta, b^{-1}\right) \in K_{p, q}-L_{p, q}$. Then we proceed further by examining the formula defining the element $\omega_{n}^{12}$ of $\widehat{G}$ and determining hereby its homomorphic image $\widehat{\chi}_{00}^{\pi}\left(\omega_{n}^{12}\right)$ in $K_{p, q}$. We thus observe that

$$
\begin{aligned}
& \widehat{\chi}_{00}^{\pi}\left(\omega_{n}^{12}\right)=\left[\widehat{\chi}_{00}^{\pi}\left(\omega_{n}^{11}\right),{ }^{n} r_{12},{ }^{n} r_{12}{ }^{n} r_{13}^{-1},{ }^{n} r_{12}{ }^{n} r_{14}^{-1},{ }^{n} r_{12}{ }^{n} r_{15}^{-1},{ }^{n} r_{12}{ }^{n} r_{16}^{-1},{ }^{n} r_{12}{ }^{n} r_{17}^{-1}\right. \text {, } \\
& \left.{ }^{n} r_{12}{ }^{n} r_{n 2}^{-1},{ }^{n} r_{12}{ }^{n} r_{n-12}^{-1},{ }^{n} r_{12}{ }^{n} r_{n-22}^{-1},{ }^{n} r_{12}{ }^{n} r_{n-32}^{-1},{ }^{n} r_{12}{ }^{n} r_{n-42}^{-1},{ }^{n} r_{12}{ }^{n} r_{11}^{2},{ }^{n} r_{12}{ }^{n} r_{22}^{2}\right] \\
& =[\widehat{\chi}_{00}^{\pi}\left(\omega_{n}^{11}\right),\left(\Delta, b^{-1}\right), \underbrace{\left(\Delta, b^{-1}\right), \ldots,\left(\Delta, b^{-1}\right)}_{10},(\Delta, b),(\Delta, b)] \in L_{p, q}-\{\Im\},
\end{aligned}
$$

again by Claim 4.1, since $\widehat{\chi}_{00}^{\pi}\left(\omega_{n}^{11}\right) \in L_{p, q}-\{\Im\}$ and $\left(\Delta, b^{-1}\right),(\Delta, b) \in K_{p, q}-L_{p, q}$. In this manner, we can continue further inspecting the formulas determining the subsequent elements $\widehat{\chi}_{00}^{\pi}\left(\omega_{n}^{22}\right), \widehat{\chi}_{00}^{\pi}\left(\omega_{n}^{23}\right), \ldots, \widehat{\chi}_{00}^{\pi}\left(\omega_{n}^{n-1 n}\right), \widehat{\chi}_{00}^{\pi}\left(\omega_{n}^{n n}\right)$ of $K_{p, q}$, until we get to the element $\widehat{\chi}_{00}^{\pi}\left(\omega_{n}^{n 1}\right)=\widehat{\chi}_{00}^{\pi}\left(\omega_{n}\right)$. In this way, we arrive at last to the conclusion that $\widehat{\chi}_{00}^{\pi}\left(\omega_{n}\right) \in L_{p, q}-\{\Im\}$, as claimed above.

ClAIM 5.4. For arbitrary prime numbers $s, t \geqslant 7$ such that $s \neq t$, the identity $w_{t}^{2} \bumpeq w_{t}$ is satisfied in the Rees matrix semigroup $\mathcal{M}\left(J_{s}, K_{p, q}, J_{s} ; R_{s}\right)$. 
Proof. Let $s, t \geqslant 7$ be prime numbers such that $s \neq t$. Since $w_{t} \in I U(X)$ and $h\left(w_{t}\right)=$ $z=\mathrm{t}\left(w_{t}\right)$, according to Corollary 2.12 , in order to show that the identity $w_{t}^{2} \bumpeq w_{t}$ is satisfied in the semigroup $\mathcal{M}\left(J_{s}, K_{p, q}, J_{s} ; R_{s}\right)$, we need to verify that, for every $k, \ell \in J_{s}$ and for every mapping $\pi: X \rightarrow J_{s} \times J_{s}$ such that $\pi(z)=(k, \ell)$, we have $\hat{\chi}_{\ell k}^{\pi}\left(\mathrm{m}\left(w_{t}\right)\right)=\mathfrak{s}$ in $K_{p, q}$ where $\widehat{\chi}_{\ell k}^{\pi}: \widehat{G} \rightarrow K_{p, q}$ is the homomorphism defined as in section 2 in the text preceding Corollary 2.12. Since $w_{t}=\gamma\left(\omega_{t}\right)$, we have $\mathrm{m}\left(w_{t}\right)=\omega_{t}$ in this condition. Thus we need to check that, for every $k, \ell \in J_{s}$ and for every mapping $\pi: X \rightarrow J_{s} \times J_{s}$ such that $\pi(z)=(k, \ell)$, we have $\widehat{\chi}_{\ell k}^{\pi}\left(\omega_{t}\right)=\mathfrak{\Im}$. Let, in this situation, $\mu, v: X \rightarrow J_{s}$ be the mappings determined by the formula $\pi(x)=(\mu(x), v(x))$, for all $x \in X$. Remember that the sandwich matrix $R_{s}$ is of the form $R_{s}=\left({ }^{s} r_{i j}\right)_{i, j \in J_{s}}$ where the entries ${ }^{s} r_{i j}$ are the elements of $K_{p, q}$ specified in the previous section. Recall now from section 2 that then the homomorphism $\widehat{\chi}_{\ell k}^{\pi}: \widehat{G} \rightarrow K_{p, q}$ is defined in such a way that, for every $x, y \in X$, we have $\widehat{\chi}_{\ell k}^{\pi}\left(p_{x y}\right)={ }^{s} r_{v(x) k}^{-1}{ }^{s} r_{\nu(x) \mu(y)}{ }^{s} r_{\ell \mu(y)}^{-1}{ }^{s} r_{\ell k}$. That is, for every $x, y \in X$, the homomorphism $\hat{\chi}_{\ell k}^{\pi}$ assigns to the element $p_{x y}$ the entry of the normalized sandwich matrix $\bar{R}_{s}^{\ell k}$ occurring in the position $(v(x), \mu(y))$. Indeed, in accordance with the description of the procedure of normalization of the sandwich matrices which has been reviewed in section 2 in the text following Proposition 2.10, the sandwich matrix $\bar{R}_{s}^{\ell k}$ which is normalized at the pair $(\ell, k)$ is of the form $\bar{R}_{s}^{\ell k}=\left({ }^{s} r_{i k}^{-1}{ }^{s} r_{i j}{ }^{s} r_{\ell j}^{-1}{ }^{s} r_{\ell k}\right)_{i, j \in J_{s}}$. Thus the homomorphism $\hat{\chi}_{\ell k}^{\pi}$ behaves as stated above. (The conditions $\mu(z)=k, v(z)=\ell$ coming from $\pi(z)=(k, \ell)$ ensure that this determination of $\widehat{\chi}_{\ell k}^{\pi}$ is all right even if $x=z$ or $y=z$.)

In the course of the verification of the condition formulated in the previous paragraph, namely that for every $k, \ell \in J_{s}$ and for every mapping $\pi: X \rightarrow J_{s} \times J_{s}$ such that $\pi(z)=(k, \ell)$, the equality $\widehat{\chi}_{\ell k}^{\pi}\left(\omega_{t}\right)=\Im$ holds, we will have to distinguish several cases corresponding to various values of the indices $k, \ell$ and we will have to discuss them separately.

\section{Assume that $k=0, \ell=0$.}

Then the matrix $\bar{R}_{s}^{\ell k}$, that is, the matrix $\bar{R}_{s}^{00}$ is just the matrix $R_{s}, \pi(z)=(0,0)$, and, for all $x, y \in X$, we have $\widehat{\chi}_{\ell k}^{\pi}\left(p_{x y}\right)=\widehat{\chi}_{00}^{\pi}\left(p_{x y}\right)={ }^{s} r_{v(x) \mu(y)}$, since the matrix $R_{s}$ is normalized at $(0,0)$. Consider now the element $\hat{\chi}_{00}^{\pi}\left(\omega_{t}\right)$ of the group $K_{p, q}$ and the elements of this group emerging consecutively during the calculation of the element $\widehat{\chi}_{00}^{\pi}\left(\omega_{t}\right)$ in accordance with the sequential definition of the element $\omega_{t}$ of the group $\widehat{G}$. Suppose, by contradiction, that $\widehat{\chi}_{00}^{\pi}\left(\omega_{t}\right) \neq \mathfrak{\Im}$. This means that $\widehat{\chi}_{00}^{\pi}\left(\omega_{t}^{t 1}\right) \neq \mathfrak{\Im}$. Hence, for every $\kappa=1,2, \ldots, t$, it follows that $\widehat{\chi}_{00}^{\pi}\left(\omega_{t}^{\kappa \kappa}\right) \neq \mathfrak{s}$, and for every $\kappa=2, \ldots, t$, it follows that $\widehat{\chi}_{00}^{\pi}\left(\omega_{t}^{\kappa-1 \kappa}\right) \neq \Im$. Now note that, for every $\kappa \in\{1,2, \ldots, t\}$, the element ${ }^{s} r_{v\left(x_{\kappa}\right) \mu\left(x_{\kappa}\right)}$ appears as the second element in the commutator determining $\widehat{\chi}_{00}^{\pi}\left(\omega_{t}^{\kappa \kappa}\right)$, and that, for every $\kappa \in\{2, \ldots, t\}$, the element ${ }^{s} r_{\nu\left(x_{\kappa-1}\right) \mu\left(x_{\kappa}\right)}$ appears as the second element in the commutator determining $\widehat{\chi}_{00}^{\pi}\left(\omega_{t}^{\kappa-1 \kappa}\right)$. Also the element ${ }^{s} r_{\nu\left(x_{t}\right) \mu\left(x_{1}\right)}$ appears as the second element in the commutator determining $\widehat{\chi}_{00}^{\pi}\left(\omega_{t}^{t 1}\right)$. Consequently, according to Claim 4.1, for every $\kappa \in\{1,2, \ldots, t\}$, we have ${ }^{s} r_{v\left(x_{\kappa}\right) \mu\left(x_{\kappa}\right)} \in K_{p, q}-L_{p, q}$, further for every $\kappa \in\{2, \ldots, t\}$, we have ${ }^{s} r_{v\left(x_{\kappa-1}\right) \mu\left(x_{\kappa}\right)} \in K_{p, q}-L_{p, q}$, and we also have ${ }^{s} r_{v\left(x_{t}\right) \mu\left(x_{1}\right)} \in$ $K_{p, q}-L_{p, q}$. Therefore, in view of the form of the sandwich matrix $R_{s}$ described in the previous section, each of the elements just mentioned must be equal either to $(\mathbb{I}, b)$ or to $\left(\Delta, b^{-1}\right)$. Furthermore, for every $\kappa \in\{1,2, \ldots, t\}$, the elements ${ }^{s} r_{v\left(x_{\kappa}\right) \mu\left(x_{\kappa}\right)}{ }^{s} r_{v\left(x_{\kappa+1}\right) \mu\left(x_{\kappa}\right)}^{-1}$ and ${ }^{s} r_{\nu\left(x_{\kappa}\right) \mu\left(x_{\kappa}\right)}{ }^{s} r_{\nu\left(x_{\kappa}\right) \mu\left(x_{\kappa-1}\right)}^{-1}$ appear, respectively, as the third and the eighth elements in the commutator determining $\widehat{\chi}_{00}^{\pi}\left(\omega_{t}^{\kappa \kappa}\right)$. (The values $\kappa+1$ and $\kappa-1$ occur within the interval $1,2, \ldots, t$ and they are computed modulo $t$, if necessary.) Therefore, according to Claim 4.1 again, these elements must all belong to the set $K_{p, q}-L_{p, q}$, 
and hence, in particular, none of these elements can be equal to $\mathfrak{\Im}$. That is, for every $\kappa \in\{1,2, \ldots, t\}$, we have ${ }^{s} r_{v\left(x_{\kappa}\right) \mu\left(x_{\kappa}\right)}{ }^{s} r_{\nu\left(x_{\kappa+1}\right) \mu\left(x_{\kappa}\right)}^{-1} \neq \mathfrak{s}$ and ${ }^{s} r_{v\left(x_{\kappa}\right) \mu\left(x_{\kappa}\right)}{ }^{s} r_{v\left(x_{\kappa}\right) \mu\left(x_{\kappa-1}\right)}^{-1} \neq \mathfrak{I}$, so that ${ }^{s} r_{v\left(x_{\kappa}\right) \mu\left(x_{\kappa}\right)} \neq{ }^{s} r_{v\left(x_{\kappa+1}\right) \mu\left(x_{\kappa}\right)}$ and ${ }^{s} r_{v\left(x_{\kappa}\right) \mu\left(x_{\kappa}\right)} \neq{ }^{s} r_{v\left(x_{\kappa}\right) \mu\left(x_{\kappa-1}\right)}$. This entails that, for all $\kappa \in\{2, \ldots, t\}$, we have $\mu\left(x_{\kappa-1}\right) \neq \mu\left(x_{\kappa}\right)$, and we also have $\mu\left(x_{t}\right) \neq \mu\left(x_{1}\right)$, and further, for all $\kappa \in\{2, \ldots, t\}$ again, we have $v\left(x_{\kappa-1}\right) \neq v\left(x_{\kappa}\right)$, and we also have $v\left(x_{t}\right) \neq v\left(x_{1}\right)$. Returning to the previous conclusion regarding the elements ${ }^{s} r_{\nu\left(x_{\kappa}\right) \mu\left(x_{\kappa}\right)}$, for all $\kappa \in$ $\{1,2, \ldots, t\}$, we remember, in particular, that we may have either ${ }^{s} r_{\nu\left(x_{1}\right) \mu\left(x_{1}\right)}=(\mathbb{I}, b)$ or ${ }^{s} r_{v\left(x_{1}\right) \mu\left(x_{1}\right)}=\left(\Delta, b^{-1}\right)$. If ${ }^{s} r_{v\left(x_{1}\right) \mu\left(x_{1}\right)}=(\mathbb{I}, b)$, then we further have ${ }^{s} r_{v\left(x_{1}\right) \mu\left(x_{2}\right)}=\left(\Delta, b^{-1}\right)$, ${ }^{s} r_{v\left(x_{2}\right) \mu\left(x_{2}\right)}=(\mathbb{I}, b),{ }^{s} r_{v\left(x_{2}\right) \mu\left(x_{3}\right)}=\left(\Delta, b^{-1}\right)$, and so on, since there is no other way that the inequalities $\mu\left(x_{\kappa-1}\right) \neq \mu\left(x_{\kappa}\right)$ and $v\left(x_{\kappa-1}\right) \neq v\left(x_{\kappa}\right)$ just deduced together with the previous conclusions concerning the elements ${ }^{s} r_{v\left(x_{k-1}\right) \mu\left(x_{k}\right)}$ and ${ }^{s} r_{v\left(x_{\kappa}\right) \mu\left(x_{k}\right)}$ can be satisfied at once. For the same reason, we then also have ${ }^{s} r_{v\left(x_{t}\right) \mu\left(x_{1}\right)}=\left(\Delta, b^{-1}\right),{ }^{s} r_{v\left(x_{t}\right) \mu\left(x_{t}\right)}=(\boldsymbol{I}, b)$, and so on. Looking at the form of the sandwich matrix $R_{s}$ once again, we see that, in this case, we therefore necessarily have

$$
\begin{aligned}
& \mu\left(x_{1}\right)=\varepsilon, \mu\left(x_{2}\right)=\varepsilon+1, \mu\left(x_{3}\right)=\varepsilon+2, \ldots \text { and } \\
& \nu\left(x_{1}\right)=\varepsilon, \nu\left(x_{2}\right)=\varepsilon+1, \nu\left(x_{3}\right)=\varepsilon+2, \ldots \text { for some } \varepsilon \in\{1,2, \ldots, s\}
\end{aligned}
$$

(This time, these values occur within the interval $1,2, \ldots, s$ and they are computed modulo $s$.) However, at the same time, coming out of the above considerations, we see that we inevitably have

$$
\begin{aligned}
& \mu\left(x_{t}\right)=\varepsilon-1, \mu\left(x_{t-1}\right)=\varepsilon-2, \ldots \text { and } \\
& \nu\left(x_{t}\right)=\varepsilon-1, \nu\left(x_{t-1}\right)=\varepsilon-2, \ldots .
\end{aligned}
$$

(These values are likewise computed modulo $s$ within $1,2, \ldots, s$.) However, the length of the above two sequences which is equal to $t$ cannot be divisible by $s$, since $s$ and $t$ are distinct prime numbers. Consequently, the equalities displayed last cannot be fulfilled if the sets of equalities displayed previously have to be satisfied. This yields a contradiction. Next we examine the possibility that ${ }^{s} r_{\nu\left(x_{1}\right) \mu\left(x_{1}\right)}=\left(\Delta, b^{-1}\right)$. Arguing as in the previous case, we find that now we further have ${ }^{s} r_{v\left(x_{1}\right) \mu\left(x_{2}\right)}=(\mathbb{I}, b)$, ${ }^{s} r_{v\left(x_{2}\right) \mu\left(x_{2}\right)}=\left(\Delta, b^{-1}\right),{ }^{s} r_{v\left(x_{2}\right) \mu\left(x_{3}\right)}=(\mathbb{I}, b)$, and so on, and, at the same time, we also have ${ }^{s} r_{v\left(x_{t}\right) \mu\left(x_{1}\right)}=(\mathbb{I}, b),{ }^{s} r_{v\left(x_{t}\right) \mu\left(x_{t}\right)}=\left(\Delta, b^{-1}\right)$, and so on. Thus, looking at the sandwich matrix $R_{s}$ once more, we see that, in this case, we necessarily have

$$
\begin{aligned}
& \mu\left(x_{1}\right)=\varepsilon+1, \mu\left(x_{2}\right)=\varepsilon, \mu\left(x_{3}\right)=\varepsilon-1, \ldots \text { and } \\
& \nu\left(x_{1}\right)=\varepsilon, \nu\left(x_{2}\right)=\varepsilon-1, \nu\left(x_{3}\right)=\varepsilon-2, \ldots \text { for some } \varepsilon \in\{1,2, \ldots, s\} .
\end{aligned}
$$

(As before, these values again occur within the interval $1,2, \ldots, s$ and they are computed modulo $s$.) However, we likewise see that we inevitably have

$$
\begin{aligned}
& \mu\left(x_{t}\right)=\varepsilon+2, \mu\left(x_{t-1}\right)=\varepsilon+3, \ldots \text { and } \\
& \nu\left(x_{t}\right)=\varepsilon+1, \nu\left(x_{t-1}\right)=\varepsilon+2, \ldots .
\end{aligned}
$$

(These values are again computed modulo $s$ within $1,2, \ldots, s$.) But since $s$ and $t$ are distinct prime numbers, and so $s$ does not divide $t$, analogous considerations as before show that the two sets of equalities displayed above cannot be fulfilled simultaneously. This again leads to a contradiction, confirming thus that $\widehat{\chi}_{00}^{\pi}\left(\omega_{t}\right)=\Im$ holds, as required. 


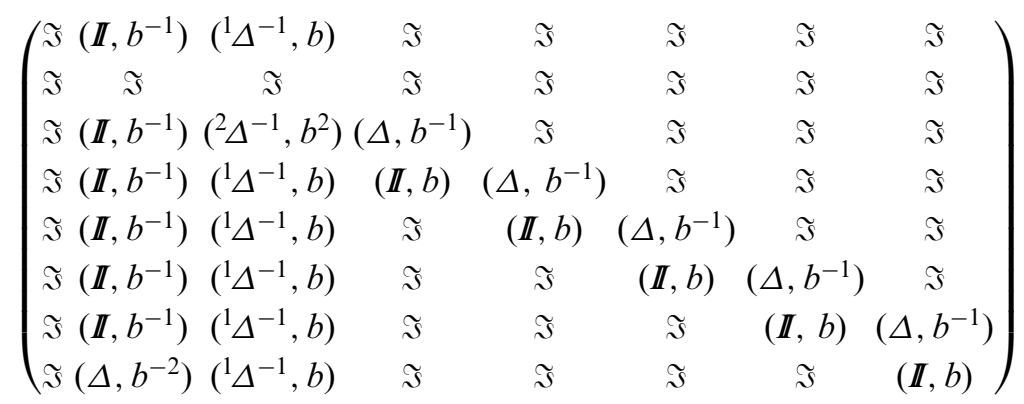

Figure 2. The sandwich matrix $\bar{R}_{7}^{10}$.

II. Assume that $k=0, \ell \neq 0$.

Notice, in this case, that by rearranging those rows and columns of the sandwich matrices $R_{s}$ and $\bar{R}_{s}^{\ell k}=\bar{R}_{s}^{\ell 0}$ which are not indexed by 0 simultaneously in such a way that the rows indexed by $\ell, \ell+1, \ldots, s$ are placed first in their respective order and only after that the rows indexed by $1,2, \ldots, \ell-1$ are placed according to their order, and subsequently also the columns indexed by $\ell, \ell+1, \ldots, s$ are placed first in their respective order and after that the columns indexed by $1,2, \ldots, \ell-1$ are placed according to their order, the matrix $R_{s}$ remains entirely unaltered, while the value of the index $\ell$ changes into 1 by this modification. This means that it suffices to examine only the situation when $k=0$ and $\ell=1$. Then $\pi(z)=(0,1)$, the sandwich matrix $\bar{R}_{s}^{\ell 0}=\bar{R}_{s}^{10}$ is normalized at the pair $(1,0)$ and it is of the form $\bar{R}_{s}^{10}=\left({ }^{s} r_{i j}{ }^{s} r_{1 j}^{-1}\right)_{i, j \in J_{s}}$, since the matrix $R_{s}$ is normalized at $(0,0)$. Recall from the first paragraph of this proof that, for every $x, y \in X$, the homomorphism $\widehat{\chi}_{\ell 0}^{\pi}$, that is (with $\ell=1$ ), the homomorphism $\widehat{\chi}_{10}^{\pi}$ assigns to the element $p_{x y}$ the entry of the sandwich matrix $\bar{R}_{s}^{10}$ occurring in the position $(v(x), \mu(y))$. Thus, it will be convenient to gain an overview of the entries of this matrix $\bar{R}_{s}^{10}$. For short, let us write ${ }^{s} \hat{r}_{i j}={ }^{s} r_{i j}{ }^{s} r_{1 j}^{-1}$, for all $i, j \in J_{s}$. Then we can write $\bar{R}_{s}^{10}=\left({ }^{s} \hat{r}_{i j}\right)_{i, j \in J_{s}}$ and, for all $x, y \in X$, we have $\hat{\chi}_{10}^{\pi}\left(p_{x y}\right)={ }^{s} \hat{r}_{v(x) \mu(y)}$. For $s=7$ the sandwich matrix $\bar{R}_{s}^{10}$ is shown in Figure 2. For general values of the prime number $s$, we have

$$
\begin{aligned}
{ }^{s} \hat{r}_{i 1} & =\left(\mathbb{I}, b^{-1}\right) \quad \text { for } i=0 \text { and for all } i=2, \ldots, s-1, \\
{ }^{s} \hat{r}_{i 2} & =\left({ }^{1} \Delta^{-1}, b\right) \quad \text { for } i=0 \text { and for all } i=3, \ldots, s, \\
s & \text { for all } i=3, \ldots, s, \\
{ }^{s} \hat{r}_{i i} & =(\mathbb{I}, b) \quad \text { for all } i=2, \ldots, s-1, \\
{ }^{s} \hat{r}_{i i+1} & =\left(\Delta, b^{-1}\right) \quad \text { and } \\
{ }^{s} \hat{r}_{22} & =\left({ }^{2} \Delta^{-1}, b^{2}\right),{ }^{s} \hat{r}_{s 1}=\left(\Delta, b^{-2}\right), \quad \\
{ }^{s} \hat{r}_{i j} & =\Im \quad \text { otherwise. }
\end{aligned}
$$

As in the previous case, suppose, by contradiction, that $\widehat{\chi}_{10}^{\pi}\left(\omega_{t}\right) \neq \Im$. As before, this means that $\widehat{\chi}_{10}^{\pi}\left(\omega_{t}^{t 1}\right) \neq \Im$, whence, for every $\kappa=1,2, \ldots, t$, it follows that $\widehat{\chi}_{10}^{\pi}\left(\omega_{t}^{\kappa \kappa}\right) \neq \Im$, and for every $\kappa=2, \ldots, t$, it follows that $\widehat{\chi}_{10}^{\pi}\left(\omega_{t}^{\kappa-1 \kappa}\right) \neq \mathfrak{\Im}$. Hence, arguing as in the previous case, but using the entries of the sandwich matrix $\bar{R}_{s}^{10}$ instead of the respective entries of the matrix $R_{s}$, on the basis of Claim 4.1, we come to the conclusion that, for every $\kappa \in\{1,2, \ldots, t\}$, we have ${ }^{s} \hat{r}_{\nu\left(x_{\kappa}\right) \mu\left(x_{\kappa}\right)} \in K_{p, q}-L_{p, q}$, for every $\kappa \in\{2, \ldots, t\}$, 
we have ${ }^{s} \hat{r}_{v\left(x_{\kappa-1}\right) \mu\left(x_{\kappa}\right)} \in K_{p, q}-L_{p, q}$, and, additionally, we have ${ }^{s} \hat{r}_{v\left(x_{t}\right) \mu\left(x_{1}\right)} \in K_{p, q}-L_{p, q}$. In the same manner as before, we further deduce that, for all $\kappa \in\{2, \ldots, t\}$, we have $\mu\left(x_{\kappa-1}\right) \neq \mu\left(x_{\kappa}\right)$ and $v\left(x_{\kappa-1}\right) \neq v\left(x_{\kappa}\right)$, and we also have $\mu\left(x_{t}\right) \neq \mu\left(x_{1}\right)$ and $v\left(x_{t}\right) \neq v\left(x_{1}\right)$. Consider next any $\kappa \in\{1,2, \ldots, t\}$. Notice that then the elements ${ }^{s} \hat{r}_{v\left(x_{\kappa}\right) \mu\left(x_{\kappa}\right)}{ }^{s} \hat{r}_{v\left(x_{\kappa+1}\right) \mu\left(x_{\kappa}\right)}^{-1},{ }^{s} \hat{r}_{v\left(x_{\kappa}\right) \mu\left(x_{\kappa}\right)}{ }^{s} \hat{r}_{v\left(x_{\kappa+2}\right) \mu\left(x_{\kappa}\right)}^{-1}$ and ${ }^{s} \hat{r}_{v\left(x_{\kappa}\right) \mu\left(x_{\kappa}\right)}{ }^{s} \hat{r}_{v\left(x_{\kappa+3}\right) \mu\left(x_{\kappa}\right)}^{-1}$ appear as the third, fourth and fifth elements in the commutator which determines $\widehat{\chi}_{10}^{\pi}\left(\omega_{t}^{\kappa \kappa}\right)$. (The values $\kappa+1, \kappa+2$ and $\kappa+3$ occur again within the interval $1,2, \ldots, t$ and they are computed modulo $t$, if necessary.) Consequently, according to Claim 4.1, none of these three elements can occur in the subgroup $L_{p, q}$, and hence, in particular, none of them can be equal to $\mathfrak{I}$. Therefore, the element ${ }^{s} \hat{r}_{\nu\left(x_{k}\right) \mu\left(x_{k}\right)}$ must be distinct from each of the elements ${ }^{s} \hat{r}_{v\left(x_{\kappa+1}\right) \mu\left(x_{\kappa}\right)},{ }^{s} \hat{r}_{v\left(x_{\kappa+2}\right) \mu\left(x_{\kappa}\right)}$ and ${ }^{s} \hat{r}_{v\left(x_{\kappa+3}\right) \mu\left(x_{\kappa}\right)}$. This entails that $v\left(x_{\kappa}\right)$ must differ from $v\left(x_{\kappa+1}\right), v\left(x_{\kappa+2}\right)$ and also from $v\left(x_{\kappa+3}\right)$. Now, going through the same deliberations as above, but, this time, having the commutator determining $\widehat{\chi}_{10}^{\pi}\left(\omega_{t}^{\kappa+1} \kappa+1\right)$ in view instead of that determining $\widehat{\chi}_{10}^{\pi}\left(\omega_{t}^{\kappa \kappa}\right)$, we likewise deduce that $v\left(x_{\kappa+1}\right)$ must differ from $v\left(x_{\kappa+2}\right)$ and also from $v\left(x_{\kappa+3}\right)$. (The values $\kappa+1, \kappa+2, \kappa+3$, and similar values appearing as indices in the elements occurring in the commutator which determines $\hat{\chi}_{10}^{\pi}\left(\omega_{t}^{\kappa+1 \kappa+1}\right)$ all belong to the interval $1,2, \ldots, t$ again and they are computed modulo $t$, if necessary.) Repeating this reasoning once again, but, this time, with the commutator determining $\widehat{\chi}_{10}^{\pi}\left(\omega_{t}^{\kappa+2} \kappa+2\right)$, we come to the conclusion that also $v\left(x_{\kappa+2}\right)$ must differ from $v\left(x_{\kappa+3}\right)$. Thus, altogether, we see that $v\left(x_{\kappa}\right), v\left(x_{\kappa+1}\right)$, $v\left(x_{\kappa+2}\right)$ and $v\left(x_{\kappa+3}\right)$ must be pairwise different. Now notice that, in the column of the sandwich matrix $\bar{R}_{s}^{10}$ indexed by 1 , there is a single element $\Im$, a single element $\left(\Delta, b^{-2}\right)$, and all remaining elements in this column are equal to $\left(\mathbb{I}, b^{-1}\right)$. Therefore, if $\mu\left(x_{\kappa}\right)=1$ for some $\kappa \in\{1,2, \ldots, t\}$, then at least one of the elements ${ }^{s} \hat{r}_{v\left(x_{\kappa+1}\right) \mu\left(x_{\kappa}\right)},{ }^{s} \hat{r}_{v\left(x_{\kappa+2}\right) \mu\left(x_{\kappa}\right)}$ and ${ }^{s} \hat{r}_{\nu\left(x_{\kappa+3}\right) \mu\left(x_{\kappa}\right)}$ must be equal to $\left(\mathbb{I}, b^{-1}\right)$. Consequently, the element ${ }^{s} \hat{r}_{\nu\left(x_{\kappa}\right) \mu\left(x_{\kappa}\right)}$ must be distinct from $\left(\mathbb{I}, b^{-1}\right)$, since, as we have seen above, it must be distinct from each of the elements ${ }^{s} \hat{r}_{\nu\left(x_{\kappa+1}\right) \mu\left(x_{\kappa}\right)},{ }^{s} \hat{r}_{v\left(x_{\kappa+2}\right) \mu\left(x_{\kappa}\right)}$ and ${ }^{s} \hat{r}_{v\left(x_{\kappa+3}\right) \mu\left(x_{\kappa}\right)}$. Turning next to the column of the matrix $\bar{R}_{s}^{10}$ indexed by 2 , in which column there is a single element $\mathfrak{s}$, a single element $\left({ }^{2} \Delta^{-1}, b^{2}\right)$, and all other elements are equal to $\left({ }^{1} \Delta^{-1}, b\right)$, analogous reasonings show that if $\mu\left(x_{\kappa}\right)=2$ for some $\kappa \in\{1,2, \ldots, t\}$, then the element ${ }^{s} \hat{r}_{\nu\left(x_{\kappa}\right) \mu\left(x_{\kappa}\right)}$ must be distinct from $\left({ }^{1} \Delta^{-1}, b\right)$. Consider further any $\kappa \in\{2, \ldots, t\}$ and notice that the elements ${ }^{s} \hat{r}_{v\left(x_{\kappa-1}\right) \mu\left(x_{\kappa}\right)}{ }^{s} \hat{r}_{v\left(x_{\kappa-2}\right) \mu\left(x_{\kappa}\right)}^{-1},{ }^{s} \hat{r}_{v\left(x_{\kappa-1}\right) \mu\left(x_{\kappa}\right)}{ }^{s} \hat{r}_{v\left(x_{\kappa-3}\right) \mu\left(x_{\kappa}\right)}^{-1}$ and ${ }^{s} \hat{r}_{v\left(x_{\kappa-1}\right) \mu\left(x_{\kappa}\right)}{ }^{s} \hat{r}_{v\left(x_{\kappa-4}\right) \mu\left(x_{\kappa}\right)}^{-1}$ appear as the eighth, ninth and tenth elements in the commutator which determines $\hat{\chi}_{10}^{\pi}\left(\omega_{t}^{\kappa-1 \kappa}\right)$. (The values $\kappa-2, \kappa-3$ and $\kappa-4$ occur again within the interval $1,2, \ldots, t$ and they are computed modulo $t$, if necessary.) Since the fact that $v\left(x_{\kappa}\right), v\left(x_{\kappa+1}\right), v\left(x_{\kappa+2}\right)$ and $v\left(x_{\kappa+3}\right)$ must be pairwise different has been deduced above for arbitrary $\kappa \in\{1,2, \ldots, t\}$, we see that also $v\left(x_{\kappa-1}\right), v\left(x_{\kappa-2}\right), v\left(x_{\kappa-3}\right)$ and $v\left(x_{\kappa-4}\right)$ must be pairwise different, having now our present $\kappa \in\{2, \ldots, t\}$ in mind. Arguing further analogously as above, we hence deduce that if $\mu\left(x_{\kappa}\right)=1$ for some $\kappa \in\{2, \ldots, t\}$, then the element ${ }^{s} \hat{r}_{v\left(x_{\kappa-1}\right) \mu\left(x_{\kappa}\right)}$ must be distinct from $\left(\mathbb{I}, b^{-1}\right)$, and if $\mu\left(x_{\kappa}\right)=2$ for some $\kappa \in\{2, \ldots, t\}$, then the element ${ }^{s} \hat{r}_{v\left(x_{\kappa-1}\right) \mu\left(x_{\kappa}\right)}$ must be distinct from $\left({ }^{1} \Delta^{-1}, b\right)$. Finally, considering similarly the elements occurring in the commutator which determines $\widehat{\chi}_{10}^{\pi}\left(\omega_{t}^{t 1}\right)$, we likewise obtain that if $\mu\left(x_{1}\right)=1$ then the element ${ }^{s} \hat{r}_{\nu\left(x_{t}\right) \mu\left(x_{1}\right)}$ must be distinct from $\left(\mathbb{I}, b^{-1}\right)$, and if $\mu\left(x_{1}\right)=2$ then the element ${ }^{s} \hat{r}_{v\left(x_{t}\right) \mu\left(x_{1}\right)}$ must be distinct from $\left({ }^{1} \Delta^{-1}, b\right)$. In this way, we find that each of the elements ${ }^{s} \hat{r}_{v\left(x_{\kappa}\right) \mu\left(x_{\kappa}\right)}$, for $\kappa \in\{1,2, \ldots, t\}$, each of the elements ${ }^{s} \hat{r}_{v\left(x_{\kappa-1}\right) \mu\left(x_{\kappa}\right)}$, for $\kappa \in\{2, \ldots, t\}$, and also the element ${ }^{s} \hat{r}_{v\left(x_{t}\right) \mu\left(x_{1}\right)}$ must be equal either to $(\mathbb{I}, b)$ or to $\left(\Delta, b^{-1}\right)$, or possibly to $\left({ }^{2} \Delta^{-1}, b^{2}\right)$ or to $\left(\Delta, b^{-2}\right)$. Remember, in this context, that we have seen above that, for all $\kappa \in\{2, \ldots, t\}$, we have $v\left(x_{\kappa-1}\right) \neq v\left(x_{\kappa}\right)$, 
and we also have $v\left(x_{t}\right) \neq v\left(x_{1}\right)$. Therefore, if for some $\kappa \in\{1,2, \ldots, t\}$, we had ${ }^{s} \hat{r}_{v\left(x_{\kappa}\right) \mu\left(x_{\kappa}\right)}=\left({ }^{2} \Delta^{-1}, b^{2}\right)$, then $\mu\left(x_{\kappa}\right)=2$ and we inevitably would have ${ }^{s} \hat{r}_{v\left(x_{\kappa-1}\right) \mu\left(x_{\kappa}\right)}=\mathfrak{s}$ or ${ }^{s} \hat{r}_{\nu\left(x_{\kappa-1}\right) \mu\left(x_{\kappa}\right)}=\left({ }^{1} \Delta^{-1}, b\right)$ if $\kappa \in\{2, \ldots, t\}$, and we would have ${ }^{s} \hat{r}_{\nu\left(x_{t}\right) \mu\left(x_{1}\right)}=\Im$ or ${ }^{s} \hat{r}_{\nu\left(x_{t}\right) \mu\left(x_{1}\right)}=\left({ }^{1} \Delta^{-1}, b\right)$ if $\kappa=1$, which has been excluded above. Similarly, if for some $\kappa \in\{1,2, \ldots, t\}$, we had ${ }^{s} \hat{r}_{\nu\left(x_{\kappa}\right) \mu\left(x_{\kappa}\right)}=\left(\Delta, b^{-2}\right)$, then $\mu\left(x_{\kappa}\right)=1$ and we inevitably would have ${ }^{s} \hat{r}_{v\left(x_{\kappa-1}\right) \mu\left(x_{\kappa}\right)}=\mathfrak{\Im}$ or ${ }^{s} \hat{r}_{v\left(x_{\kappa-1}\right) \mu\left(x_{\kappa}\right)}=\left(\mathbb{I}, b^{-1}\right)$ if $\kappa \in\{2, \ldots, t\}$, and we would have ${ }^{s} \hat{r}_{v\left(x_{t}\right) \mu\left(x_{1}\right)}=\mathfrak{J}$ or ${ }^{s} \hat{r}_{v\left(x_{t}\right) \mu\left(x_{1}\right)}=\left(\mathbb{I}, b^{-1}\right)$ if $\kappa=1$, which has also been excluded above. Thus, for every $\kappa \in\{1,2, \ldots, t\}$, we necessarily must have either ${ }^{s} \hat{r}_{v\left(x_{\kappa}\right) \mu\left(x_{\kappa}\right)}=(\mathbb{I}, b)$ or ${ }^{s} \hat{r}_{v\left(x_{\kappa}\right) \mu\left(x_{\kappa}\right)}=\left(\Delta, b^{-1}\right)$. Very similar arguments show that, for every $\kappa \in\{2, \ldots, t\}$, we also must necessarily have either ${ }^{s} \hat{r}_{\nu\left(x_{k-1}\right) \mu\left(x_{\kappa}\right)}=(\mathbb{I}, b)$ or ${ }^{s} \hat{r}_{v\left(x_{k-1}\right) \mu\left(x_{\kappa}\right)}=\left(\Delta, b^{-1}\right)$. And finally, for the same reasons, we may actually have only either ${ }^{s} \hat{r}_{v\left(x_{t}\right) \mu\left(x_{1}\right)}=(\mathbb{I}, b)$ or ${ }^{s} \hat{r}_{\nu\left(x_{t}\right) \mu\left(x_{1}\right)}=\left(\Delta, b^{-1}\right)$. So that, in particular, we may have either ${ }^{s} \hat{r}_{\nu\left(x_{1}\right) \mu\left(x_{1}\right)}=(\mathbb{I}, b)$ or ${ }^{s} \hat{r}_{v\left(x_{1}\right) \mu\left(x_{1}\right)}=\left(\Delta, b^{-1}\right)$. If ${ }^{s} \hat{r}_{v\left(x_{1}\right) \mu\left(x_{1}\right)}=(\mathbb{I}, b)$, then, similarly as in the previous case, we hence further get that ${ }^{s} \hat{r}_{v\left(x_{1}\right) \mu\left(x_{2}\right)}=\left(\Delta, b^{-1}\right),{ }^{s} \hat{r}_{v\left(x_{2}\right) \mu\left(x_{2}\right)}=(\mathbb{I}, b),{ }^{s} \hat{r}_{v\left(x_{2}\right) \mu\left(x_{3}\right)}=\left(\Delta, b^{-1}\right)$, and so on. Looking now at the sandwich matrix $\bar{R}_{s}^{10}$, we see that, consequently, this time we must have

$$
\begin{aligned}
\mu\left(x_{1}\right)=\varepsilon, \mu\left(x_{2}\right)=\varepsilon+1, \mu\left(x_{3}\right)=\varepsilon+2, \ldots, & \mu\left(x_{t}\right)=\varepsilon+t-1, \text { and } \\
\nu\left(x_{1}\right)=\varepsilon, \nu\left(x_{2}\right)=\varepsilon+1, \nu\left(x_{3}\right)=\varepsilon+2, \ldots, & v\left(x_{t}\right)=\varepsilon+t-1, \\
& \text { for some } \varepsilon \in\{3, \ldots, s-t+1\},
\end{aligned}
$$

provided that $s \geqslant t+2$. Otherwise the above conditions are impossible to fulfill. However, at the same time, for the same reasons as before, we further get that ${ }^{s} \hat{r}_{v\left(x_{t}\right) \mu\left(x_{1}\right)}=\left(\Delta, b^{-1}\right)$ and ${ }^{s} \hat{r}_{v\left(x_{t}\right) \mu\left(x_{t}\right)}=(\mathbb{I}, b)$, which means that we must also have $\mu\left(x_{t}\right)=\varepsilon-1$ and $v\left(x_{t}\right)=\varepsilon-1$. By the way, this enforces that $\varepsilon \geqslant 4$. But, above all, this is incompatible with the previous findings about $\mu\left(x_{t}\right)$ and $v\left(x_{t}\right)$, leading thus to a contradiction. Next we examine the possibility that ${ }^{s} \hat{r}_{\nu\left(x_{1}\right) \mu\left(x_{1}\right)}=\left(\Delta, b^{-1}\right)$. Then, similarly as in the previous case again, from the above notes we further deduce that ${ }^{s} \hat{r}_{v\left(x_{1}\right) \mu\left(x_{2}\right)}=(\mathbb{I}, b),{ }^{s} \hat{r}_{v\left(x_{2}\right) \mu\left(x_{2}\right)}=\left(\Delta, b^{-1}\right),{ }^{s} \hat{r}_{v\left(x_{2}\right) \mu\left(x_{3}\right)}=(\mathbb{I}, b)$, and so on. Looking at the sandwich matrix $\bar{R}_{s}^{10}$ once again, we see that, this time, we are led to the conclusion that we must have

$$
\begin{aligned}
& \mu\left(x_{1}\right)=\varepsilon+1, \mu\left(x_{2}\right)=\varepsilon, \mu\left(x_{3}\right)=\varepsilon-1, \ldots, \mu\left(x_{t}\right)=\varepsilon-t+2 \text {, and } \\
& \nu\left(x_{1}\right)=\varepsilon, \nu\left(x_{2}\right)=\varepsilon-1, \nu\left(x_{3}\right)=\varepsilon-2, \ldots, \nu\left(x_{t}\right)=\varepsilon-t+1 \text {, } \\
& \text { for some } \varepsilon \in\{t+1, \ldots, s-1\} \text {, }
\end{aligned}
$$

provided that $s \geqslant t+2$. Otherwise the above conditions are again impossible to fulfill. However, at the same time, from the above notes we further obtain that ${ }^{s} \hat{r}_{v\left(x_{t}\right) \mu\left(x_{1}\right)}=$ $(\mathbb{I}, b)$ and ${ }^{s} \hat{r}_{\nu\left(x_{t}\right) \mu\left(x_{t}\right)}=\left(\Delta, b^{-1}\right)$, which now means that we must also have $\mu\left(x_{t}\right)=$ $\varepsilon+2$ and $v\left(x_{t}\right)=\varepsilon+1$. Besides, this requires that $\varepsilon \leqslant s-2$. But, first of all, this is again incompatible with the preceding findings about $\mu\left(x_{t}\right)$ and $v\left(x_{t}\right)$, yielding thus a contradiction as previously. This contradiction verifies that $\hat{\chi}_{10}^{\pi}\left(\omega_{t}\right)=\mathfrak{s}$ holds, as required.

III. Assume that $k \neq 0, \ell=0$.

Proceeding in exactly the same way as at the beginning of the previous case, that is, rearranging the rows and the columns of the sandwich matrices $R_{s}$ and $\bar{R}_{s}^{\ell k}=\bar{R}_{s}^{0 k}$ appropriately, we again find that it suffices to examine only the situation when $k=1$ and $\ell=0$, for instance. Then $\pi(z)=(1,0)$, the sandwich matrix $\bar{R}_{s}^{0 k}=\bar{R}_{s}^{01}$ is normalized 


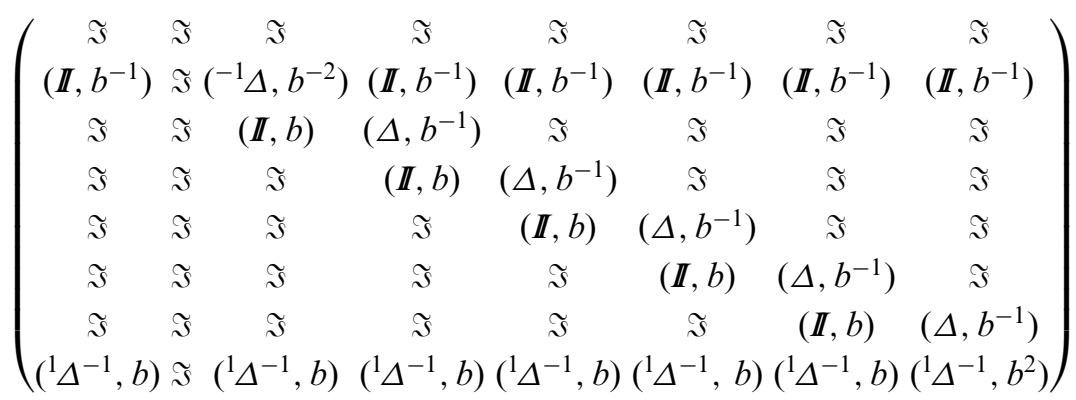

Figure 3. The sandwich matrix $\bar{R}_{7}^{01}$.

at the pair $(0,1)$ and it is of the form $\bar{R}_{s}^{01}=\left({ }^{s} r_{i 1}^{-1}{ }^{s} r_{i j}\right)_{i, j \in J_{s}}$. Remember once again that, for every $x, y \in X$, the homomorphism $\hat{\chi}_{0 k}^{\pi}$, that is (with $k=1$ ), the homomorphism $\widehat{\chi}_{01}^{\pi}$ assigns to the element $p_{x y}$ the entry of the sandwich matrix $\bar{R}_{s}^{01}$ occurring in the position $(v(x), \mu(y))$. This is the reason why this sandwich matrix is dealt with. It is again appropriate to inspect the entries of this matrix $\bar{R}_{s}^{01}$. However, it is fairly easy to realize that the form of this matrix is very much like that of the matrix $\bar{R}_{s}^{10}$ in the previous case. Essentially, except for some insignificant details, only the roles of the rows and the columns in these matrices are interchanged. For $s=7$ the sandwich matrix $\bar{R}_{s}^{01}$ is shown in Figure 3 . Hence it is easy to understand how this matrix looks for general values of the prime number $s$. Thus this case can be treated in quite the same manner as the previous one. Working with the rows of the sandwich matrix $\bar{R}_{s}^{01}$ rather than with the columns, as opposed to the previous case, and taking the form of the commutators determining the elements $\widehat{\chi}_{01}^{\pi}\left(\omega_{t}^{11}\right), \widehat{\chi}_{01}^{\pi}\left(\omega_{t}^{12}\right), \widehat{\chi}_{01}^{\pi}\left(\omega_{t}^{22}\right)$, $\widehat{\chi}_{01}^{\pi}\left(\omega_{t}^{23}\right), \ldots, \widehat{\chi}_{01}^{\pi}\left(\omega_{t}^{t-1 t}\right), \widehat{\chi}_{01}^{\pi}\left(\omega_{t}^{t t}\right)$, and $\widehat{\chi}_{01}^{\pi}\left(\omega_{t}^{t 1}\right)=\widehat{\chi}_{01}^{\pi}\left(\omega_{t}\right)$ into account, in the same way as before, we find that the assumption that $\widehat{\chi}_{01}^{\pi}\left(\omega_{t}\right) \neq \mathfrak{\Im}$ again leads to a contradiction. Thus $\widehat{\chi}_{01}^{\pi}\left(\omega_{t}\right)=\mathfrak{s}$ holds, as required.

IV. Assume finally that $k \neq 0, \ell \neq 0$.

Notice that in this case, one can also simplify the calculations by rearranging the rows and the columns of the sandwich matrices $R_{s}$ and $\bar{R}_{s}^{\ell k}$ appropriately. Namely, similarly as before, by rearranging those rows and columns of these matrices which are not indexed by 0 simultaneously in such a way that the columns indexed by $k, k+1, \ldots, s$ are placed first in their respective order and only after that the columns indexed by $1,2, \ldots, k-1$ are placed according to their order, and subsequently also the rows indexed by $k, k+1, \ldots, s$ are placed first in their respective order and after that the columns indexed by $1,2, \ldots, k-1$ are placed according to their order, the matrix $R_{s}$ remains entirely unaltered, while the value of the index $k$ changes into 1 and the value of the index $\ell$ changes either into $\ell-k+1$ if $k \leqslant \ell$ or into $\ell+s-k+1$ if $k>\ell$, by this modification. This reasoning reveals that it is enough to examine only the situation when $k=1$. Nevertheless, we have still to distinguish several subcases according to possible values of the index $\ell$.

IV.i. Assume that $k=1$ and that $2<\ell<s-1$. Then $\pi(z)=(1, \ell)$, the sandwich matrix $\bar{R}_{s}^{\ell 1}$ is normalized at the pair $(\ell, 1)$ and, as ${ }^{s} r_{\ell 1}=\Im$ for $2<\ell<s-1$, it is of the form $\bar{R}_{s}^{\ell 1}=\left({ }^{s} r_{i 1}^{-1}{ }^{s} r_{i j}{ }^{s} r_{\ell j}^{-1}\right)_{i, j \in J_{s}}$. Recall once more from the first paragraph of this proof that, for every $x, y \in X$, the homomorphism $\hat{\chi}_{\ell 1}^{\pi}$ assigns to the element $p_{x y}$ the entry of the sandwich matrix $\bar{R}_{s}^{\ell 1}$ occurring in the position 


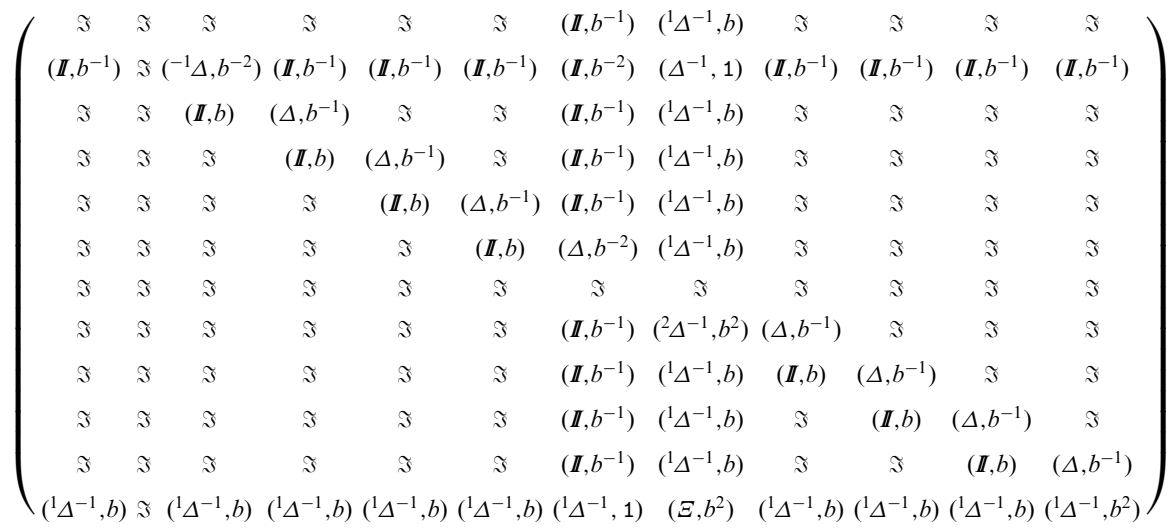

Figure 4. The sandwich matrix $\bar{R}_{11}^{61}$, where $\Xi={ }^{1} \Delta^{-1} \cdot{ }^{2} \Delta^{-1}$.

$(v(x), \mu(y))$. Thus, as before, it will be convenient to gain an overview of the entries of this matrix $\bar{R}_{s}^{\ell 1}$. For short, let us write ${ }^{s} \bar{r}_{i j}={ }^{s} r_{i 1}^{-1}{ }^{s} r_{i j} s r_{\ell j}^{-1}$, for all $i, j \in J_{s}$. Then we can write $\bar{R}_{s}^{\ell 1}=\left({ }^{s} \bar{r}_{i j}\right)_{i, j \in J_{s}}$ and, for all $x, y \in X$, we have $\widehat{\chi}_{\ell 1}^{\pi}\left(p_{x y}\right)={ }^{s} \bar{r}_{v(x) \mu(y)}$. For $s=11$ and $\ell=6$ the sandwich matrix $\bar{R}_{s}^{\ell 1}$ is shown in Figure 4 . For general values of the prime number $s$, in view of the assumption that $2<\ell<s-1$, we have

$$
\begin{aligned}
{ }^{s} \bar{r}_{1 j} & =\left(\mathbb{I}, b^{-1}\right) \quad \text { for } j=0 \text { and for all } j=3, \ldots, \ell-1, \ell+2, \ldots, s, \\
{ }^{s} \bar{r}_{i \ell} & =\left(\mathbb{I}, b^{-1}\right) \quad \text { for } i=0 \text { and for all } i=2, \ldots, \ell-2, \ell+1, \ldots, s-1, \\
{ }^{s} \bar{r}_{s j} & =\left({ }^{1} \Delta^{-1}, b\right) \quad \text { for } j=0 \text { and for all } j=2, \ldots, \ell-1, \ell+2, \ldots, s-1, \\
{ }^{s} \bar{r}_{i \ell+1} & =\left({ }^{1} \Delta^{-1}, b\right) \quad \text { for } i=0 \text { and for all } i=2, \ldots, \ell-1, \ell+2, \ldots, s-1, \\
{ }^{s} \bar{r}_{i i} & =(\mathbb{I}, b) \quad \text { for all } i=2, \ldots, \ell-1, \ell+2, \ldots, s-1, \\
{ }^{s} \bar{r}_{i i+1} & =\left(\Delta, b^{-1}\right) \quad \text { for all } i=2, \ldots, \ell-2, \ell+1, \ldots, s-1 \\
{ }^{s} \bar{r}_{12} & =\left({ }^{-1} \Delta, b^{-2}\right), \quad{ }^{s} \bar{r}_{1 \ell}=\left(\mathbb{I}, b^{-2}\right), \quad{ }^{s} \bar{r}_{1 \ell+1}=\left(\Delta^{-1}, 1\right), \\
{ }^{s} \bar{r}_{\ell-1 \ell} & =\left(\Delta, b^{-2}\right),{ }^{s} \bar{r}_{\ell+1 \ell+1}=\left({ }^{2} \Delta^{-1}, b^{2}\right), \\
{ }^{s} \bar{r}_{s \ell} & =\left({ }^{1} \Delta^{-1}, 1\right), \quad{ }^{s} \bar{r}_{s \ell+1}=\left({ }^{1} \Delta^{-1} \cdot{ }^{2} \Delta^{-1}, b^{2}\right),{ }^{s} \bar{r}_{s s}=\left({ }^{1} \Delta^{-1}, b^{2}\right), \quad \text { and } \\
{ }^{s} \bar{r}_{i j} & =\Im \quad \text { otherwise. }
\end{aligned}
$$

As in the preceding cases, we again proceed by contradiction. Thus suppose that $\widehat{\chi}_{\ell 1}^{\pi}\left(\omega_{t}\right) \neq \Im$. This means that $\widehat{\chi}_{\ell 1}^{\pi}\left(\omega_{t}^{t 1}\right) \neq \Im$, which entails that $\widehat{\chi}_{\ell 1}^{\pi}\left(\omega_{t}^{\kappa \kappa}\right) \neq \Im$, for all $\kappa=1,2, \ldots, t$, and that $\widehat{\chi}_{\ell 1}^{\pi}\left(\omega_{t}^{\kappa-1 \kappa}\right) \neq \Im$, for all $\kappa=2, \ldots, t$. Thus, arguing as in case I, but using the entries of the sandwich matrix $\bar{R}_{s}^{\ell 1}$ instead of the respective entries of the matrix $R_{s}$, we again arrive at the conclusion based on Claim 4.1 saying that, for every $\kappa \in\{1,2, \ldots, t\}$, we have ${ }^{s} \bar{r}_{\nu\left(x_{\kappa}\right) \mu\left(x_{\kappa}\right)} \in K_{p, q}-L_{p, q}$, for every $\kappa \in\{2, \ldots, t\}$, we have ${ }^{s} \bar{r}_{v\left(x_{\kappa-1}\right) \mu\left(x_{\kappa}\right)} \in K_{p, q}-L_{p, q}$, and we also have ${ }^{s} \bar{r}_{v\left(x_{t}\right) \mu\left(x_{1}\right)} \in K_{p, q}-L_{p, q}$. In the same manner as in case I, we next deduce that, for all $\kappa \in\{2, \ldots, t\}$, we have $\mu\left(x_{\kappa-1}\right) \neq$ $\mu\left(x_{\kappa}\right)$ and $v\left(x_{\kappa-1}\right) \neq v\left(x_{\kappa}\right)$, and we also have $\mu\left(x_{t}\right) \neq \mu\left(x_{1}\right)$ and $v\left(x_{t}\right) \neq v\left(x_{1}\right)$. Our further thoughts will be akin to those encountered in case II. Consider any $\kappa \in$ $\{1,2, \ldots, t\}$. Notice that then the elements ${ }^{s} \bar{r}_{v\left(x_{\kappa}\right) \mu\left(x_{\kappa}\right)}{ }^{s} \bar{r}_{v\left(x_{\kappa+1}\right) \mu\left(x_{\kappa}\right)}^{-1},{ }^{s} \bar{r}_{v\left(x_{\kappa}\right) \mu\left(x_{\kappa}\right)}{ }^{s} \bar{r}_{v\left(x_{\kappa+2}\right) \mu\left(x_{\kappa}\right)}^{-1}$, ${ }^{s} \bar{r}_{\nu\left(x_{\kappa}\right) \mu\left(x_{\kappa}\right)}{ }^{s} \bar{r}_{v\left(x_{\kappa+3}\right) \mu\left(x_{\kappa}\right)}^{-1},{ }^{s} \bar{r}_{v\left(x_{\kappa}\right) \mu\left(x_{\kappa}\right)}{ }^{s} \bar{r}_{v\left(x_{\kappa+4}\right) \mu\left(x_{\kappa}\right)}^{-1}$ and ${ }^{s} \bar{r}_{v\left(x_{\kappa}\right) \mu\left(x_{\kappa}\right)}{ }^{s} \bar{r}_{v\left(x_{\kappa+5}\right) \mu\left(x_{\kappa}\right)}^{-1}$ appear as the third up to the seventh elements in the commutator which determines $\chi_{\ell 1}^{\pi}\left(\omega_{t}^{\kappa \kappa}\right)$. (The values 
$\kappa+1, \kappa+2, \kappa+3, \kappa+4$ and $\kappa+5$ occur again within the interval $1,2, \ldots, t$ and they are computed modulo $t$, if necessary.) Consequently, in view of Claim 4.1, none of these five elements can occur in the subgroup $L_{p, q}$, and hence, in particular, none of them can be equal to $\mathfrak{\Im}$. Therefore, the element ${ }^{s} \bar{r}_{\nu\left(x_{k}\right) \mu\left(x_{\kappa}\right)}$ must be distinct from each of the elements ${ }^{s} \bar{r}_{\nu\left(x_{\kappa+1}\right) \mu\left(x_{\kappa}\right)},{ }^{s} \bar{r}_{\nu\left(x_{k+2}\right) \mu\left(x_{k}\right)},{ }^{s} \bar{r}_{\nu\left(x_{k+3}\right) \mu\left(x_{k}\right)},{ }^{s} \bar{r}_{\nu\left(x_{k+4}\right) \mu\left(x_{k}\right)}$ and ${ }^{s} \bar{r}_{v\left(x_{k+5}\right) \mu\left(x_{k}\right)}$. This entails that $v\left(x_{\kappa}\right)$ must differ from each of $v\left(x_{\kappa+1}\right), v\left(x_{\kappa+2}\right), v\left(x_{\kappa+3}\right), v\left(x_{\kappa+4}\right)$ and $v\left(x_{\kappa+5}\right)$. Now, repeating the same deliberations as above, but having consecutively in view the commutators determining $\widehat{\chi}_{\ell 1}^{\pi}\left(\omega_{t}^{\kappa+1 \kappa+1}\right), \widehat{\chi}_{\ell 1}^{\pi}\left(\omega_{t}^{\kappa+2 \kappa+2}\right), \widehat{\chi}_{\ell 1}^{\pi}\left(\omega_{t}^{\kappa+3 \kappa+3}\right), \widehat{\chi}_{\ell 1}^{\pi}\left(\omega_{t}^{\kappa+4 \kappa+4}\right)$ and $\widehat{\chi}_{\ell 1}^{\pi}\left(\omega_{t}^{\kappa+5 \kappa+5}\right)$ instead of the one determining $\widehat{\chi}_{\ell 1}^{\pi}\left(\omega_{t}^{\kappa \kappa}\right)$, similarly as in case II, we eventually come to the conclusion that $v\left(x_{\kappa}\right), v\left(x_{\kappa+1}\right), v\left(x_{\kappa+2}\right), v\left(x_{\kappa+3}\right), v\left(x_{\kappa+4}\right)$ and $v\left(x_{\kappa+5}\right)$ must be pairwise different. (The values $\kappa+1, \kappa+2, \kappa+3, \kappa+4, \kappa+5$, and similar values appearing as indices in the elements occurring in the commutators just mentioned all belong to the interval $1,2, \ldots, t$ and they are computed modulo $t$, as before, if necessary.) Next consider any $\kappa \in\{1,2, \ldots, t\}$ once again and return once more to the above deliberations concerning the commutator which determines $\widehat{\chi}_{\ell 1}^{\pi}\left(\omega_{t}^{\kappa \kappa}\right)$, but, this time, having the elements ${ }^{s} \bar{r}_{v\left(x_{\kappa}\right) \mu\left(x_{\kappa}\right)}{ }^{s} \bar{r}_{v\left(x_{\kappa}\right) \mu\left(x_{\kappa-1}\right)}^{-1},{ }^{s} \bar{r}_{v\left(x_{\kappa}\right) \mu\left(x_{\kappa}\right)}{ }^{s} \bar{r}_{v\left(x_{\kappa}\right) \mu\left(x_{\kappa-2}\right)}^{-1}$, ${ }^{s} \bar{r}_{v\left(x_{\kappa}\right) \mu\left(x_{\kappa}\right)}{ }^{s} \bar{r}_{v\left(x_{\kappa}\right) \mu\left(x_{\kappa-3}\right)}^{-1},{ }^{s} \bar{r}_{v\left(x_{\kappa}\right) \mu\left(x_{\kappa}\right)}{ }^{s} \bar{r}_{v\left(x_{\kappa}\right) \mu\left(x_{\kappa-4}\right)}^{-1}$ and ${ }^{s} \bar{r}_{\nu\left(x_{\kappa}\right) \mu\left(x_{\kappa}\right)}{ }^{s} \bar{r}_{v\left(x_{\kappa}\right) \mu\left(x_{\kappa-5}\right)}^{-1}$ in view, which appear as the eighth up to the twelfth elements in this commutator. (The values $\kappa-1, \kappa-2, \kappa-3, \kappa-4$ and $\kappa-5$ occur again within the interval $1,2, \ldots, t$ and they are computed modulo $t$, if necessary.) In view of Claim 4.1 again, none of these five elements can be equal to $\mathfrak{\Im}$. Therefore, the element ${ }^{s} \bar{r}_{\nu\left(x_{k}\right) \mu\left(x_{k}\right)}$ must be distinct from each of the elements ${ }^{s} \bar{r}_{v\left(x_{k}\right) \mu\left(x_{\kappa-1}\right)},{ }^{s} \bar{r}_{v\left(x_{k}\right) \mu\left(x_{\kappa-2}\right)}{ }^{s} \bar{r}_{v\left(x_{k}\right) \mu\left(x_{\kappa-3}\right)},{ }^{s} \bar{r}_{v\left(x_{\kappa}\right) \mu\left(x_{\kappa-4}\right)}$ and ${ }^{s} \bar{r}_{v\left(x_{k}\right) \mu\left(x_{k-5}\right)}$. Pursuing farther these reasonings in quite the same way as above, and repeating them subsequently, but, this time, with the commutators determining consecutively $\widehat{\chi}_{\ell 1}^{\pi}\left(\omega_{t}^{\kappa-1 \kappa-1}\right), \widehat{\chi}_{\ell 1}^{\pi}\left(\omega_{t}^{\kappa-2 \kappa-2}\right), \widehat{\chi}_{\ell 1}^{\pi}\left(\omega_{t}^{\kappa-3 \kappa-3}\right), \widehat{\chi}_{\ell 1}^{\pi}\left(\omega_{t}^{\kappa-4 \kappa-4}\right)$ and $\widehat{\chi}_{\ell 1}^{\pi}\left(\omega_{t}^{\kappa-5 \kappa-5}\right)$ in place of the one determining $\hat{\chi}_{\ell 1}^{\pi}\left(\omega_{t}^{\kappa \kappa}\right)$, we eventually come to the conclusion that also $\mu\left(x_{\kappa}\right)$, $\mu\left(x_{\kappa-1}\right), \mu\left(x_{\kappa-2}\right), \mu\left(x_{\kappa-3}\right), \mu\left(x_{\kappa-4}\right)$ and $\mu\left(x_{\kappa-5}\right)$ must be pairwise different. (Once again, as before, the values $\kappa-1, \kappa-2, \kappa-3, \kappa-4, \kappa-5$, and similar values appearing as indices in the elements occurring in the commutators just mentioned all belong to the interval $1,2, \ldots, t$ and they are computed modulo $t$, if necessary.) Now observe that, in the column of the sandwich matrix $\bar{R}_{s}^{\ell 1}$ indexed by $\ell$, there is a single element $\Im$, a single element $\left({ }^{1} \Delta^{-1}, 1\right)$, a single element $\left(\mathbb{I}, b^{-2}\right)$, a single element $\left(\Delta, b^{-2}\right)$, and all remaining elements in this column are equal to $\left(\mathbb{I}, b^{-1}\right)$. Therefore, if $\mu\left(x_{\kappa}\right)=\ell$ for some $\kappa \in\{1,2, \ldots, t\}$, then at least one of the five elements ${ }^{s} \bar{r}_{v\left(x_{\kappa+1}\right) \mu\left(x_{\kappa}\right)},{ }^{s} \bar{r}_{v\left(x_{\kappa+2}\right) \mu\left(x_{\kappa}\right)}$, ${ }^{s} \bar{r}_{\nu\left(x_{\kappa+3}\right) \mu\left(x_{\kappa}\right)},{ }^{s} \bar{r}_{v\left(x_{\kappa+4}\right) \mu\left(x_{\kappa}\right)}$ and ${ }^{s} \bar{r}_{\nu\left(x_{\kappa+5}\right) \mu\left(x_{\kappa}\right)}$ must be equal to $\left(\mathbb{I}, b^{-1}\right)$. Consequently, the element ${ }^{s} \bar{r}_{\nu\left(x_{\kappa}\right) \mu\left(x_{\kappa}\right)}$ must be distinct from $\left(\boldsymbol{I}, b^{-1}\right)$, since, as we have seen above, it must be distinct from each of the five elements just named. Quite similarly, in the column of the matrix $\bar{R}_{s}^{\ell 1}$ indexed by $\ell+1$, there is a single element $\Im$, a single element $\left(\Delta^{-1}, 1\right)$, a single element $\left({ }^{2} \Delta^{-1}, b^{2}\right)$, a single element $\left({ }^{1} \Delta^{-1} \cdot{ }^{2} \Delta^{-1}, b^{2}\right)$, and all other elements in this column are equal to $\left({ }^{1} \Delta^{-1}, b\right)$. Thus, analogous arguments as above reveal that if $\mu\left(x_{\kappa}\right)=\ell+1$ for some $\kappa \in\{1,2, \ldots, t\}$, then the element ${ }^{s} \bar{r}_{v\left(x_{\kappa}\right) \mu\left(x_{\kappa}\right)}$ must be distinct from $\left({ }^{1} \Delta^{-1}, b\right)$. Next turn to the rows of the sandwich matrix $\bar{R}_{s}^{\ell 1}$. Notice that, in the row of this matrix indexed by 1 , there is a single element $\mathfrak{s}$, a single element $\left(\Delta^{-1}, 1\right)$, a single element $\left(\mathbb{I}, b^{-2}\right)$, a single element $\left({ }^{-1} \Delta, b^{-2}\right)$, and all other elements in this row are equal to $\left(\mathbb{I}, b^{-1}\right)$. Therefore, if $v\left(x_{\kappa}\right)=1$ for some $\kappa \in\{1,2, \ldots, t\}$, then at least one of the five elements ${ }^{s} \bar{r}_{v\left(x_{\kappa}\right) \mu\left(x_{\kappa-1}\right)},{ }^{s} \bar{r}_{v\left(x_{\kappa}\right) \mu\left(x_{\kappa-2}\right)}$, ${ }^{s} \bar{r}_{v\left(x_{\kappa}\right) \mu\left(x_{\kappa-3}\right)},{ }^{s} \bar{r}_{v\left(x_{\kappa}\right) \mu\left(x_{\kappa-4}\right)}$ and ${ }^{s} \bar{r}_{v\left(x_{\kappa}\right) \mu\left(x_{\kappa-5}\right)}$ must be equal to $\left(\mathbb{I}, b^{-1}\right)$. Consequently, the element ${ }^{s} \bar{r}_{v\left(x_{\kappa}\right) \mu\left(x_{\kappa}\right)}$ must be distinct from $\left(\boldsymbol{I}, b^{-1}\right)$, since, as we have also seen above, it 
must be distinct from each of the five elements just named. Likewise, in the row of the matrix $\bar{R}_{s}^{\ell 1}$ indexed by $s$, there is a single element $\Im$, a single element $\left({ }^{1} \Delta^{-1}, 1\right)$, a single element $\left({ }^{1} \Delta^{-1}, b^{2}\right)$, a single element $\left({ }^{1} \Delta^{-1} \cdot{ }^{2} \Delta^{-1}, b^{2}\right)$, and all other elements in this row are equal to $\left({ }^{1} \Delta^{-1}, b\right)$. Thus, analogous arguments as above show that if $\nu\left(x_{\kappa}\right)=s$ for some $\kappa \in\{1,2, \ldots, t\}$, then the element ${ }^{s} \bar{r}_{\nu\left(x_{\kappa}\right) \mu\left(x_{\kappa}\right)}$ must be distinct from $\left({ }^{1} \Delta^{-1}, b\right)$. We proceed further by considering any $\kappa \in\{2, \ldots, t\}$, by observing that the elements ${ }^{s} \bar{r}_{v\left(x_{\kappa-1}\right) \mu\left(x_{\kappa}\right)}{ }^{s} \bar{r}_{v\left(x_{\kappa-2}\right) \mu\left(x_{\kappa}\right)}^{-1},{ }^{s} \bar{r}_{v\left(x_{\kappa-1}\right) \mu\left(x_{\kappa}\right)}{ }^{s} \bar{r}_{v\left(x_{\kappa-3}\right) \mu\left(x_{\kappa}\right)}^{-1},{ }^{s} \bar{r}_{v\left(x_{\kappa-1}\right) \mu\left(x_{\kappa}\right)}{ }^{s} \bar{r}_{v\left(x_{\kappa-4}\right) \mu\left(x_{\kappa}\right)}^{-1}$, ${ }^{s} \bar{r}_{\nu\left(x_{\kappa-1}\right) \mu\left(x_{\kappa}\right)}{ }^{s} \bar{r}_{\nu\left(x_{\kappa-5}\right) \mu\left(x_{\kappa}\right)}^{-1}$ together with the element ${ }^{s} \bar{r}_{\nu\left(x_{\kappa-1}\right) \mu\left(x_{\kappa}\right)}{ }^{s} \bar{r}_{\nu\left(x_{\kappa-6}\right) \mu\left(x_{\kappa}\right)}^{-1}$ appear as the eighth up to the twelfth elements in the commutator which determines $\widehat{\chi}_{\ell 1}^{\pi}\left(\omega_{t}^{\kappa-1 \kappa}\right)$, and by noting similarly that also the other elements ${ }^{s} \bar{r}_{v\left(x_{\kappa-1}\right) \mu\left(x_{\kappa}\right)}{ }^{s} \bar{r}_{v\left(x_{\kappa-1}\right) \mu\left(x_{\kappa+1}\right)}^{-1}$, ${ }^{s} \bar{r}_{v\left(x_{\kappa-1}\right) \mu\left(x_{\kappa}\right)}{ }^{s} \bar{r}_{v\left(x_{\kappa-1}\right) \mu\left(x_{\kappa+2}\right)}^{-1},{ }^{s} \bar{r}_{v\left(x_{\kappa-1}\right) \mu\left(x_{\kappa}\right)}{ }^{s} \bar{r}_{v\left(x_{\kappa-1}\right) \mu\left(x_{\kappa+3}\right)}^{-1}, \quad{ }^{s} \bar{r}_{v\left(x_{\kappa-1}\right) \mu\left(x_{\kappa}\right)}{ }^{s} \bar{r}_{v\left(x_{k-1}\right) \mu\left(x_{\kappa+4}\right)}^{-1}$ and the element ${ }^{s} \bar{r}_{v\left(x_{\kappa-1}\right) \mu\left(x_{\kappa}\right)}{ }^{s} \bar{r}_{v\left(x_{\kappa-1}\right) \mu\left(x_{\kappa+5}\right)}^{-1}$ appear as the third up to the seventh elements in the mentioned commutator. Since the facts that $v\left(x_{\kappa}\right), v\left(x_{\kappa+1}\right), v\left(x_{\kappa+2}\right), v\left(x_{\kappa+3}\right), v\left(x_{\kappa+4}\right)$ and $v\left(x_{\kappa+5}\right)$ must be pairwise different and that $\mu\left(x_{\kappa}\right), \mu\left(x_{\kappa-1}\right), \mu\left(x_{\kappa-2}\right), \mu\left(x_{\kappa-3}\right)$, $\mu\left(x_{\kappa-4}\right)$ and $\mu\left(x_{\kappa-5}\right)$ must be pairwise different have been deduced above for arbitrary $\kappa \in\{1,2, \ldots, t\}$, we see that also $v\left(x_{\kappa-1}\right), v\left(x_{\kappa-2}\right), v\left(x_{\kappa-3}\right), v\left(x_{\kappa-4}\right), v\left(x_{\kappa-5}\right)$ and $v\left(x_{\kappa-6}\right)$ must be pairwise different and that $\mu\left(x_{\kappa}\right), \mu\left(x_{\kappa+1}\right), \mu\left(x_{\kappa+2}\right), \mu\left(x_{\kappa+3}\right), \mu\left(x_{\kappa+4}\right)$ and $\mu\left(x_{\kappa+5}\right)$ must be pairwise different, having now our present $\kappa \in\{2, \ldots, t\}$ in mind. Arguing further entirely analogously as before, we hence deduce that if $\mu\left(x_{\kappa}\right)=\ell$ for some $\kappa \in\{2, \ldots, t\}$, then the element ${ }^{s} \bar{r}_{v\left(x_{\kappa-1}\right) \mu\left(x_{\kappa}\right)}$ must be distinct from $\left(\mathbb{I}, b^{-1}\right)$, if $\mu\left(x_{\kappa}\right)=\ell+1$ for some $\kappa \in\{2, \ldots, t\}$, then the element ${ }^{s} \bar{r}_{v\left(x_{\kappa-1}\right) \mu\left(x_{\kappa}\right)}$ must be distinct from $\left({ }^{1} \Delta^{-1}, b\right)$, if $v\left(x_{\kappa-1}\right)=1$ for some $\kappa \in\{2, \ldots, t\}$, then the element ${ }^{s} \bar{r}_{v\left(x_{\kappa-1}\right) \mu\left(x_{\kappa}\right)}$ must be distinct from $\left(\mathbb{I}, b^{-1}\right)$, and if $v\left(x_{\kappa-1}\right)=s$ for some $\kappa \in\{2, \ldots, t\}$, then the element ${ }^{s} \bar{r}_{\nu\left(x_{\kappa-1}\right) \mu\left(x_{\kappa}\right)}$ must be distinct from $\left({ }^{1} \Delta^{-1}, b\right)$. Finally, considering similarly the elements occurring in the commutator which determines $\widehat{\chi}_{\ell 1}^{\pi}\left(\omega_{t}^{t 1}\right)$, we likewise obtain that if $\mu\left(x_{1}\right)=\ell$ then the element ${ }^{s} \bar{r}_{v\left(x_{t}\right) \mu\left(x_{1}\right)}$ must be distinct from $\left(\mathbb{I}, b^{-1}\right)$, if $\mu\left(x_{1}\right)=\ell+1$ then the element ${ }^{s} \bar{r}_{\nu\left(x_{t}\right) \mu\left(x_{1}\right)}$ must be distinct from $\left({ }^{1} \Delta^{-1}, b\right)$, if $v\left(x_{t}\right)=1$ then the element ${ }^{s} \bar{r}_{v\left(x_{t}\right) \mu\left(x_{1}\right)}$ must be distinct from $\left(\mathbb{I}, b^{-1}\right)$, and if $v\left(x_{t}\right)=s$ then the element ${ }^{s} \bar{r}_{\nu\left(x_{t}\right) \mu\left(x_{1}\right)}$ must be distinct from $\left({ }^{1} \Delta^{-1}, b\right)$. We complete these remarks by noting that $\left(\Delta^{-1}, 1\right),\left({ }^{1} \Delta^{-1}, 1\right) \in L_{p, q}$, so that, according to Claim 4.1 again, none of the elements ${ }^{s} \bar{r}_{v\left(x_{\kappa}\right) \mu\left(x_{\kappa}\right)}$, for any $\kappa \in\{1,2, \ldots, t\}$, and none of the elements ${ }^{s} \bar{r}_{v\left(x_{\kappa-1}\right) \mu\left(x_{\kappa}\right)}$, for any $\kappa \in\{2, \ldots, t\}$, can be equal either to $\left(\Delta^{-1}, 1\right)$ or to $\left({ }^{1} \Delta^{-1}, 1\right)$. Likewise the element ${ }^{s} \bar{r}_{\nu\left(x_{t}\right) \mu\left(x_{1}\right)}$ can be equal neither to $\left(\Delta^{-1}, 1\right)$ nor to $\left({ }^{1} \Delta^{-1}, 1\right)$. But there are still two collections of elements in the sandwich matrix $\bar{R}_{s}^{\ell 1}$ which come into consideration as possible values of the elements just mentioned. Namely, they are the elements

$$
\begin{aligned}
{ }^{s} \bar{r}_{12}=\left({ }^{-1} \Delta, b^{-2}\right),{ }^{s} \bar{r}_{22}=(\mathbb{I}, b),{ }^{s} \bar{r}_{23}=\left(\Delta, b^{-1}\right),{ }^{s} \bar{r}_{33}=(\mathbb{I}, b), \\
{ }^{s} \bar{r}_{34}=\left(\Delta, b^{-1}\right), \ldots,{ }^{s} \bar{r}_{\ell-2 \ell-1}=\left(\Delta, b^{-1}\right), \\
{ }^{s} \bar{r}_{\ell-1 \ell-1}=(\mathbb{I}, b),{ }^{s} \bar{r}_{\ell-1 \ell}=\left(\Delta, b^{-2}\right),{ }^{s} \bar{r}_{1 \ell}=\left(\mathbb{I}, b^{-2}\right),
\end{aligned}
$$

and the elements

$$
\begin{array}{r}
{ }^{s} \bar{r}_{\ell+1 \ell+1}=\left({ }^{2} \Delta^{-1}, b^{2}\right),{ }^{s} \bar{r}_{\ell+1 \ell+2}=\left(\Delta, b^{-1}\right),{ }^{s} \bar{r}_{\ell+2 \ell+2}=(\mathbb{I}, b),{ }^{s} \bar{r}_{\ell+2 \ell+3}=\left(\Delta, b^{-1}\right), \\
{ }^{s} \bar{r}_{\ell+3 \ell+3}=(\mathbb{I}, b), \ldots,{ }^{s} \bar{r}_{s-1 s-1}=(\mathbb{I I}, b), \\
{ }^{s} \bar{r}_{s-1 s}=\left(\Delta, b^{-1}\right),{ }^{s} \bar{r}_{s s}=\left({ }^{1} \Delta^{-1}, b^{2}\right),{ }^{s} \bar{r}_{s} \ell+1 \\
\end{array}
$$


Thus assume, for instance, that $\left(v\left(x_{1}\right), \mu\left(x_{1}\right)\right)$ is the pair of indices of an element lying in the first of the above two collections of elements. Then we may have either $v\left(x_{1}\right)=\varepsilon$, $\mu\left(x_{1}\right)=\varepsilon$ for some $\varepsilon \in\{2, \ldots, \ell-1\}$, or $v\left(x_{1}\right)=1, \mu\left(x_{1}\right)=\ell$, or else $v\left(x_{1}\right)=\varepsilon$, $\mu\left(x_{1}\right)=\varepsilon+1$ for some $\varepsilon \in\{1,2, \ldots, \ell-1\}$. In accordance with this differentiation, we will further distinguish three possibilities. Thus, coming out of the remarks on the diversity of the values $\mu\left(x_{\kappa}\right)$ and $v\left(x_{\kappa}\right)$, for distinct $\kappa \in\{1,2, \ldots, t\}$, which have appeared earlier in the treatment of the present subcase, and arguing in a similar way to case I, we find that, in the situation when the first of the three possibilities just mentioned occurs, we necessarily must have

$$
\begin{aligned}
& \mu\left(x_{1}\right)=\varepsilon, \mu\left(x_{2}\right)=\varepsilon+1, \mu\left(x_{3}\right)=\varepsilon+2, \ldots \text { and } \\
& \nu\left(x_{1}\right)=\varepsilon, \nu\left(x_{2}\right)=\varepsilon+1, \nu\left(x_{3}\right)=\varepsilon+2, \ldots \text { for some } \varepsilon \in\{2, \ldots, \ell-1\},
\end{aligned}
$$

and, at the same time, we also inevitably must have

$$
\begin{aligned}
& \mu\left(x_{t}\right)=\varepsilon-1, \mu\left(x_{t-1}\right)=\varepsilon-2, \ldots \text { and } \\
& \nu\left(x_{t}\right)=\varepsilon-1, \nu\left(x_{t-1}\right)=\varepsilon-2, \ldots .
\end{aligned}
$$

(The values involving $\varepsilon$ are computed modulo $\ell-1$ this time and, for the mappings $\mu$ and $v$, they occur within the intervals $2, \ldots, \ell$ and $1, \ldots, \ell-1$, respectively.) Then, if $\ell-1>t$, the above two sequences whose length is $t$ are shorter then $\ell-1$, which is obviously impossible. Therefore we must have $\ell-1 \leqslant t$. (Then, in fact, $\ell-1$ divides $t$, so that $\ell-1=t$.) In this situation, however, there is $\lambda \in\{2, \ldots, t\}$ such that $\mu\left(x_{\lambda}\right)=\ell$ and $\nu\left(x_{\lambda}\right)=1$. Furthermore, then $\mu\left(x_{\lambda-1}\right)=\ell-1$ and $v\left(x_{\lambda-1}\right)=\ell-1$. Thus we get that ${ }^{s} \bar{r}_{v\left(x_{\lambda-1}\right) \mu\left(x_{\lambda}\right)}=\left(\Delta, b^{-2}\right)$ and ${ }^{s} \bar{r}_{v\left(x_{\lambda-1}\right) \mu\left(x_{\lambda-1}\right)}=(\mathbb{I}, b)$. Hence we obtain that ${ }^{s} \bar{r}_{v\left(x_{\lambda-1}\right) \mu\left(x_{\lambda}\right)}{ }^{s} \bar{r}_{v\left(x_{\lambda-1}\right) \mu\left(x_{\lambda-1}\right)}^{2}=(\Delta, 1)$, which element belongs to the subgroup $L_{p, q}$. But this element occurs as the last element but one in the commutator which determines $\widehat{\chi}_{\ell 1}^{\pi}\left(\omega_{t}^{\lambda-1 \lambda}\right)$. Consequently, by Claim 4.1, we have $\widehat{\chi}_{\ell 1}^{\pi}\left(\omega_{t}^{\lambda-1 \lambda}\right)=\mathfrak{I}$, whence we get that $\widehat{\chi}_{\ell 1}^{\pi}\left(\omega_{t}\right)=\mathfrak{\Im}$. But this is a contradiction. The situation when the second of the three possibilities mentioned above occurs, that is, when $v\left(x_{1}\right)=1$ and $\mu\left(x_{1}\right)=\ell$ holds can be treated in quite the same manner as the previous one. Namely, it suffices to take $\varepsilon=\ell$ in the previous situation. The only difference is that, in the concluding deliberations, we now have $\lambda=1$. Then $\mu\left(x_{1}\right)=\ell, v\left(x_{1}\right)=1$ and $\mu\left(x_{t}\right)=\ell-1, v\left(x_{t}\right)=\ell-1$. Thus, similarly as above, we get that ${ }^{s} \bar{r}_{\nu\left(x_{t}\right) \mu\left(x_{1}\right)}=\left(\Delta, b^{-2}\right)$ and ${ }^{s} \bar{r}_{\nu\left(x_{t}\right) \mu\left(x_{t}\right)}=(\mathbb{I}, b)$, whence we obtain that $\left.{ }^{s} \bar{r}_{v\left(x_{t}\right) \mu\left(x_{1}\right)}\right)^{s} \bar{r}_{v\left(x_{t}\right) \mu\left(x_{t}\right)}^{2}=(\Delta, 1)$. This element belongs to the subgroup $L_{p, q}$ and it occurs as the last element but one in the commutator which determines $\hat{\chi}_{\ell 1}^{\pi}\left(\omega_{t}^{t 1}\right)$, that is, $\widehat{\chi}_{\ell 1}^{\pi}\left(\omega_{t}\right)$. By Claim 4.1, this yields that $\widehat{\chi}_{\ell 1}^{\pi}\left(\omega_{t}\right)=\Im$. This is again a contradiction. In the situation when the last of the three possibilities mentioned above occurs, arguing as above, we find that, this time, we necessarily must have

$$
\begin{aligned}
& \mu\left(x_{1}\right)=\varepsilon+1, \mu\left(x_{2}\right)=\varepsilon, \mu\left(x_{3}\right)=\varepsilon-1, \ldots \text { and } \\
& \nu\left(x_{1}\right)=\varepsilon, \nu\left(x_{2}\right)=\varepsilon-1, \nu\left(x_{3}\right)=\varepsilon-2, \ldots \text { for some } \varepsilon \in\{1,2, \ldots, \ell-1\},
\end{aligned}
$$

and, at the same time, we also inevitably must have

$$
\begin{aligned}
& \mu\left(x_{t}\right)=\varepsilon+2, \mu\left(x_{t-1}\right)=\varepsilon+3, \ldots \text { and } \\
& \nu\left(x_{t}\right)=\varepsilon+1, \nu\left(x_{t-1}\right)=\varepsilon+2, \ldots .
\end{aligned}
$$

(As before, in this situation again, the values involving $\varepsilon$ are computed modulo $\ell-1$ and, for the mappings $\mu$ and $\nu$, they occur within the intervals $2, \ldots, \ell$ and 


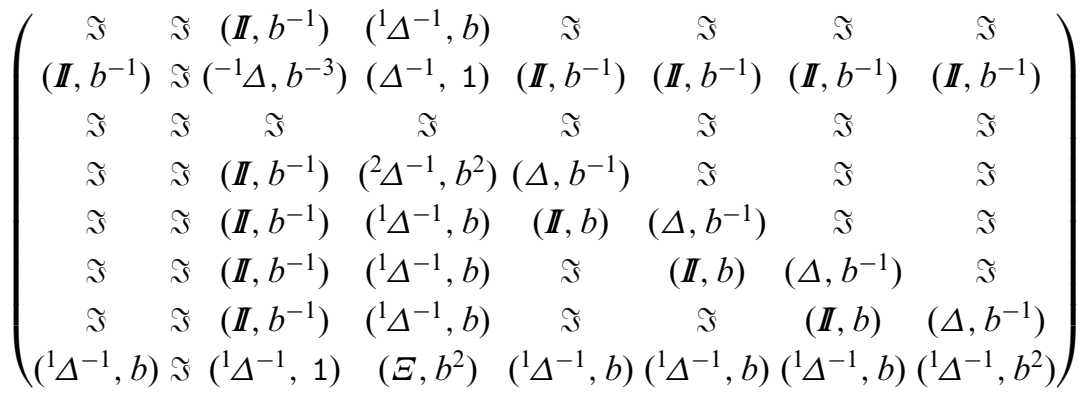

Figure 5. The sandwich matrix $\bar{R}_{7}^{21}$, where $\Xi={ }^{1} \Delta^{-1} \cdot{ }^{2} \Delta^{-1}$.

$1, \ldots, \ell-1$, respectively.) As above, it turns out that the inequality $\ell-1>t$ is impossible, so that we again have $\ell-1 \leqslant t$ (in fact, $\ell-1$ divides $t$, and hence $\ell-1=t$ ). This time, however, there is $\lambda \in\{1,2, \ldots, t\}$ such that $\mu\left(x_{\lambda+1}\right)=\ell$ and $v\left(x_{\lambda}\right)=1$. (If $\lambda=t$ then $\lambda+1$ is interpreted as 1.) Furthermore, then $\mu\left(x_{\lambda}\right)=2$ and $\nu\left(x_{\lambda-1}\right)=2$. (If $\lambda=1$ then $\lambda-1$ is interpreted as $t$.) Thus we get that ${ }^{s} \bar{r}_{\nu\left(x_{\lambda}\right) \mu\left(x_{\lambda}\right)}=\left({ }^{-1} \Delta, b^{-2}\right)$ and ${ }^{s} \bar{r}_{v\left(x_{\lambda-1}\right) \mu\left(x_{\lambda}\right)}=(\mathbb{I}, b)$, whence we obtain that ${ }^{s} \bar{r}_{\nu\left(x_{\lambda}\right) \mu\left(x_{\lambda}\right)} \bar{r}_{v\left(x_{\lambda-1}\right) \mu\left(x_{\lambda}\right)}^{2}=\left({ }^{-1} \Delta, 1\right)$. But this element belongs to the subgroup $L_{p, q}$ and, at the same time, this element also occurs as the last element but one in the commutator which determines $\widehat{\chi}_{\ell 1}^{\pi}\left(\omega_{t}^{\lambda \lambda}\right)$. Consequently, by Claim 4.1, we have $\widehat{\chi}_{\ell 1}^{\pi}\left(\omega_{t}^{\lambda \lambda}\right)=\mathfrak{s}$, whence we again get that $\widehat{\chi}_{\ell 1}^{\pi}\left(\omega_{t}\right)=\mathfrak{s}$. This is again a contradiction, as before. The treatment of the present subcase is essentially completed by these conclusions, since the situation when $\left(v\left(x_{1}\right), \mu\left(x_{1}\right)\right)$ is the pair of indices of an element lying in the second of the two collections of elements of the matrix $\bar{R}_{s}^{\ell 1}$ displayed previously in this discussion can be settled quite analogously. Thus altogether we may conclude that $\widehat{\chi}_{\ell 1}^{\pi}\left(\omega_{t}\right)=\mathfrak{s}$ holds, as required.

IV.ii. Assume that $k=1$ and $\ell=2$.

Then $\pi(z)=(1,2)$, the sandwich matrix $\bar{R}_{s}^{21}$ is normalized at the pair $(2,1)$, and ${ }^{s} r_{21}=\Im$. Notice that, consequently, the entry of the sandwich matrix $\bar{R}_{s}^{21}$ occuring in the position $(1,2)$ is

$$
{ }^{s} \bar{r}_{12}={ }^{s} r_{11}^{-1 s} r_{12}{ }^{s} r_{22}^{-1}=\left({ }^{-1} \Delta, b^{-3}\right) .
$$

With this exception, the description of the sandwich matrix $\bar{R}_{s}^{21}$ does not differ from the description of the matrix $\bar{R}_{s}^{\ell 1}$ given in the previous subcase where, this time, one takes $\ell=2$, of course. It actually means that the first of the two collections of elements of the matrix $\bar{R}_{s}^{\ell 1}$ displayed in the above text, where the previous subcase is treated, now collapses to the single element $\left({ }^{-1} \Delta, b^{-3}\right)$ shown above. The rest of the matrix $\bar{R}_{s}^{\ell 1}$ remains essentially unchanged if one takes $\ell=2$. For $s=7$ the sandwich matrix $\bar{R}_{s}^{21}$ is shown in Figure 5. Hence it should be clear how this matrix looks for general values of the prime number $s$. The treatment of this subcase proceeds, as before, by showing that the assumption that $\hat{\chi}_{21}^{\pi}\left(\omega_{t}\right) \neq \mathfrak{s}$ leads to a contradiction. The discussion confirming this fact can be performed in the same fashion as in the previous subcase. However, at the point where the two collections of elements of the matrix $\bar{R}_{s}^{\ell 1}$ are displayed in the previous discussion, the first of which now reduces to the single element $\left({ }^{-1} \Delta, b^{-3}\right)$ owing to the fact that $\ell=2$, the arguments can be partly simplified by showing that, for 


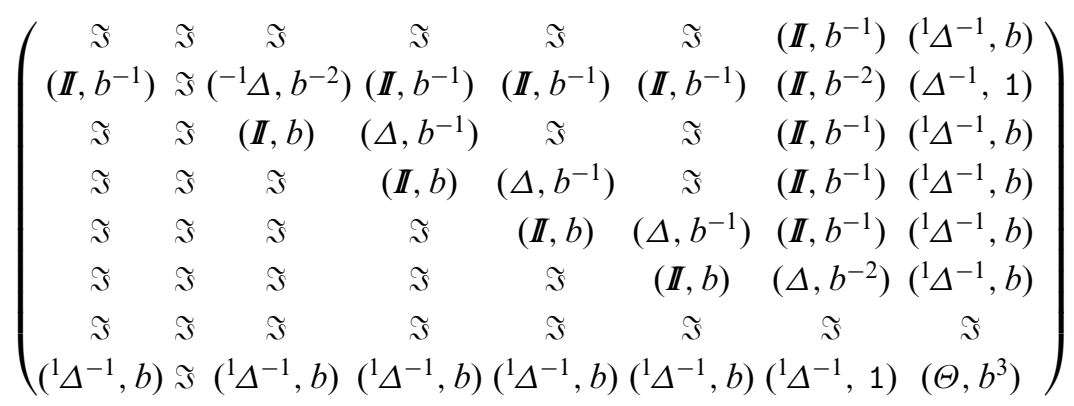

Figure 6. The sandwich matrix $\bar{R}_{7}^{61}$, where $\Theta={ }^{1} \Delta^{-1} \cdot{ }^{3} \Delta^{-1}$.

any $\kappa \in\{1,2, \ldots, t\}$, the element ${ }^{s} \bar{r}_{\nu\left(x_{\kappa}\right) \mu\left(x_{\kappa}\right)}$ cannot be equal to $\left({ }^{-1} \Delta, b^{-3}\right)$, for any $\kappa \in$ $\{2, \ldots, t\}$, the element ${ }^{s} \bar{r}_{\nu\left(x_{\kappa-1}\right) \mu\left(x_{\kappa}\right)}$ cannot be equal to $\left({ }^{-1} \Delta, b^{-3}\right)$, and also the element ${ }^{s} \bar{r}_{v\left(x_{t}\right) \mu\left(x_{1}\right)}$ cannot be equal to $\left(^{-1} \Delta, b^{-3}\right)$. For instance, if for some $\kappa \in\{1,2, \ldots, t\}$, we had ${ }^{s} \bar{r}_{\nu\left(x_{\kappa}\right) \mu\left(x_{\kappa}\right)}=\left({ }^{-1} \Delta, b^{-3}\right)$, then $\mu\left(x_{\kappa}\right)=2$, and if $\kappa \in\{2, \ldots, t\}$, we inevitably would have either ${ }^{s} \bar{r}_{\nu\left(x_{\kappa-1}\right) \mu\left(x_{\kappa}\right)}=\mathfrak{s}$ or ${ }^{s} \bar{r}_{v\left(x_{\kappa-1}\right) \mu\left(x_{\kappa}\right)}=\left({ }^{1} \Delta^{-1}, 1\right)$ or ${ }^{s} \bar{r}_{\nu\left(x_{k-1}\right) \mu\left(x_{k}\right)}=\left(\mathbb{I}, b^{-1}\right)$, since $v\left(x_{\kappa-1}\right) \neq v\left(x_{\kappa}\right)$, and if $\kappa=1$, we would have either ${ }^{s} \bar{r}_{\nu\left(x_{t}\right) \mu\left(x_{1}\right)}=\mathfrak{s}$ or ${ }^{s} \bar{r}_{v\left(x_{t}\right) \mu\left(x_{1}\right)}=$ $\left({ }^{1} \Delta^{-1}, 1\right)$ or ${ }^{s} \bar{r}_{v\left(x_{t}\right) \mu\left(x_{1}\right)}=\left(\mathbb{I I}, b^{-1}\right)$, since $v\left(x_{t}\right) \neq v\left(x_{1}\right)$, These possibilities, however, have already been excluded in the preceding discussion. Similar reasonings verify also the other assertions stated above. Consequently, in our present discussion, we have to deal only with the second of the two collections of elements of the matrix $\bar{R}_{s}^{\ell 1}$ which are displayed in the text examining the previous subcase. And this second collection of elements looks the same even when $\ell=2$. Hence the discussion can be completed in the same manner as previously, verifying thus that $\widehat{\chi}_{21}^{\pi}\left(\omega_{t}\right)=\mathfrak{s}$ holds, as required.

IV.iii. Assume that $k=1$ and $\ell=s-1$.

Then $\pi(z)=(1, s-1)$, the sandwich matrix $\bar{R}_{s}^{s-11}$ is normalized at the pair $(s-1,1)$, and ${ }^{s} r_{s-11}=\mathfrak{I}$. This time, notice that, as a consequence, the entry of the sandwich matrix $\bar{R}_{s}^{s-11}$ occuring in the position $(s, s)$ is

$$
{ }^{s} \bar{r}_{s s}={ }^{s} r_{s 1}^{-1 s} r_{s s}{ }^{s} r_{s-1 s}^{-1}=\left({ }^{1} \Delta^{-1} \cdot{ }^{3} \Delta^{-1}, b^{3}\right)
$$

With this exception, the description of the sandwich matrix $\bar{R}_{s}^{s-11}$ again does not differ from the description of the matrix $\bar{R}_{s}^{\ell 1}$ given in subcase IV.i where, this time, one takes $\ell=s-1$, of course. This time, it actually means that the second of the two collections of elements of the matrix $\bar{R}_{s}^{\ell 1}$ displayed in the preceding text, where subcase IV.i is treated, now collapses to the single element $\left({ }^{1} \Delta^{-1} \cdot{ }^{3} \Delta^{-1}, b^{3}\right)$ shown above. The rest of the matrix $\bar{R}_{s}^{\ell 1}$ remains again essentially unchanged if one takes $\ell=s-1$. For $s=7$ the sandwich matrix $\bar{R}_{s}^{s-11}$ is shown in Figure 6. Hence it is obvious how this matrix looks for general values of the prime number $s$. Just as before, this subcase is again settled by showing that the assumption that $\widehat{\chi}_{s-11}^{\pi}\left(\omega_{t}\right) \neq \Im$ leads to a contradiction. The discussion leading to this conclusion can again be done in the same manner as in subcase IV.i. Similarly as in the previous subcase, however, the reasonings can be partly simplified by showing that, this time, for any $\kappa \in\{1,2, \ldots, t\}$, the element ${ }^{s} \bar{r}_{v\left(x_{\kappa}\right) \mu\left(x_{\kappa}\right)}$ cannot be equal to $\left({ }^{1} \Delta^{-1} \cdot{ }^{3} \Delta^{-1}, b^{3}\right)$, for any $\kappa \in\{2, \ldots, t\}$, the element ${ }^{s} \bar{r}_{v\left(x_{\kappa-1}\right) \mu\left(x_{\kappa}\right)}$ cannot be equal to $\left({ }^{1} \Delta^{-1} \cdot{ }^{3} \Delta^{-1}, b^{3}\right)$, and also the element ${ }^{s} \bar{r}_{\nu\left(x_{t}\right) \mu\left(x_{1}\right)}$ cannot be equal to $\left({ }^{1} \Delta^{-1} \cdot{ }^{3} \Delta^{-1}, b^{3}\right)$. The arguments confirming these assertions are quite the same as 


\begin{tabular}{|c|c|c|c|c|c|c|}
\hline$(\mathbb{I}, b)$ & $\Im\left({ }^{1} \Delta^{-1}, b^{2}\right)$ & $(\mathbb{I I}, b)$ & $(\mathbb{I}, b)$ & $(\mathbb{I}, b)$ & $(\mathbb{I}, b)$ & $(\mathbb{I}, b)$ \\
\hline $\mathfrak{\Im}$ & $\mathfrak{I}$ & $\mathfrak{\Im}$ & $\Im$ & $\mathfrak{\Im}$ & $\mathfrak{\Im}$ & $\mathfrak{\Im}$ \\
\hline$(\mathbb{I}, b)$ & $\mathfrak{I}\left({ }^{2} \Delta^{-1}, b^{3}\right)$ & $(\Delta, 1)$ & $(\mathbb{I I}, b)$ & $(\mathbb{I}, b)$ & $(\mathbb{I}, b)$ & $(\mathbb{I}, b)$ \\
\hline$(\mathbb{I}, b)$ & $\mathfrak{I}\left({ }^{1} \Delta^{-1}, b^{2}\right)$ & $\left(\mathbb{I I}, b^{2}\right)$ & $(\Delta, 1)$ & $(\mathbb{I}, b)$ & $(\mathbb{I I}, b)$ & $(\mathbb{I}, b)$ \\
\hline$(\mathbb{I}, b)$ & $\mathfrak{I}\left({ }^{1} \Delta^{-1}, b^{2}\right)$ & $(I I, b)$ & $\left(\mathbb{I I}, b^{2}\right)$ & $(\Delta, 1)$ & $(\mathbb{I I}, b)$ & $(\mathbb{I}, b)$ \\
\hline$(\mathbb{I}, b)$ & $\mathfrak{\Im}\left({ }^{1} \Delta^{-1}, b^{2}\right)$ & $(\mathbb{I I}, b)$ & $(\boldsymbol{I I}, b)$ & $\left(\mathbb{I I}, b^{2}\right)$ & $(\Delta, 1)$ & $(\mathbb{I}, b)$ \\
\hline$(\mathbb{I}, b)$ & $\mathfrak{\Im}\left({ }^{1} \Delta^{-1}, b^{2}\right)$ & $(\mathbb{I I}, b)$ & $(\mathbb{I}, b)$ & $(\mathbb{I}, b)$ & $\left(\mathbb{I}, b^{2}\right)$ & $(\Delta, 1)$ \\
\hline
\end{tabular}

Figure 7. The sandwich matrix $\bar{R}_{7}^{11}$, where $\Xi={ }^{1} \Delta^{-1} \cdot{ }^{2} \Delta^{-1}$.

in the previous subcase. With this amendment, the entire discussion can be performed in the same way as in subcase IV.i. But, this time, only the first of the two collections of elements of the matrix $\bar{R}_{s}^{\ell 1}$ which are displayed in the text where subcase IV.i is examined has to be taken into consideration. The fact that we now have $\ell=s-1$ does not affect this collection of elements. Consequently, we can again conclude that $\widehat{\chi}_{s-11}^{\pi}\left(\omega_{t}\right)=\Im$ holds, as required.

IV.iv. Assume that $k=1$ and $\ell=1$.

Then $\pi(z)=(1,1)$, the sandwich matrix $\bar{R}_{s}^{11}$ is normalized at the pair $(1,1)$, and ${ }^{s} r_{11}=(\mathbb{I}, b)$. The sandwich matrix $\bar{R}_{s}^{11}$ is of the form $\bar{R}_{s}^{11}=\left({ }^{s} r_{i 1}^{-1}{ }^{s} r_{i j}{ }^{s} r_{1 j}^{-1}{ }^{s} r_{11}\right)_{i, j \in J_{s}}$. For short, let us write ${ }^{s} \tilde{r}_{i j}={ }^{s} r_{i 1}^{-1}{ }^{s} r_{i j}{ }^{s} r_{1 j}^{-1 s} r_{11}$. Then we can write $\bar{R}_{s}^{11}=\left({ }^{s} \tilde{r}_{i j}\right)_{i, j \in J_{s}}$. Remember once again that, for every $x, y \in X$, the homomorphism $\widehat{\chi}_{11}^{\pi}$ assigns to the element $p_{x y}$ the entry of the matrix $\bar{R}_{s}^{11}$ occurring in the position $(v(x), \mu(y))$. That is, for all $x, y \in X$, we have $\widehat{\chi}_{11}^{\pi}\left(p_{x y}\right)={ }^{s} \tilde{r}_{v(x) \mu(y)}$. Thus, as before, it will be appropriate to gain an overview of the entries of the matrix $\bar{R}_{s}^{11}$. For $s=7$ the sandwich matrix $\bar{R}_{s}^{11}$ is shown in Figure 7. For general values of the prime number $s$, we have

$$
\begin{aligned}
s \tilde{r}_{i 2} & =\left({ }^{1} \Delta^{-1}, b^{2}\right) \quad \text { for } i=0 \text { and for all } i=3, \ldots, s-1, \\
s \tilde{r}_{s j} & =\left({ }^{1} \Delta^{-1}, b^{2}\right) \quad \text { for } j=0 \text { and for all } j=3, \ldots, s-1, \\
s \tilde{r}_{i i} & =\left(\mathbb{I}, b^{2}\right) \quad \text { for all } i=3, \ldots, s-1, \\
s \tilde{r}_{i i+1} & =(\Delta, 1) \quad \text { for all } i=2, \ldots, s-1, \\
s \tilde{r}_{22} & =\left({ }^{2} \Delta^{-1}, b^{3}\right), \quad s \tilde{r}_{s 2}=\left({ }^{1} \Delta^{-1} \cdot{ }^{2} \Delta^{-1}, b^{3}\right), \quad s \tilde{r}_{s s}=\left({ }^{1} \Delta^{-1}, b^{3}\right), \\
s \tilde{r}_{i j} & =\Im \quad \text { whenever } i=1 \text { or } j=1, \quad \text { and } \\
s \tilde{r}_{i j} & =(\mathbb{I}, b) \quad \text { otherwise. }
\end{aligned}
$$

As in the preceding cases, suppose, by contradiction, that $\widehat{\chi}_{11}^{\pi}\left(\omega_{t}\right) \neq \mathfrak{s}$. Then the same arguments as in case I based on Claim 4.1 and applied to the homomorphism $\widehat{\chi}_{11}^{\pi}$ and to the entries of the sandwich matrix $\bar{R}_{s}^{11}$ show that, for every $\kappa \in\{1,2, \ldots, t\}$, we have ${ }^{s} \tilde{r}_{\nu\left(x_{\kappa}\right) \mu\left(x_{\kappa}\right)} \in K_{p, q}-L_{p, q}$, for every $\kappa \in\{2, \ldots, t\}$, we have ${ }^{s} \tilde{r}_{\nu\left(x_{\kappa-1}\right) \mu\left(x_{\kappa}\right)} \in K_{p, q}-L_{p, q}$, and we also have ${ }^{s} \tilde{r}_{v\left(x_{t}\right) \mu\left(x_{1}\right)} \in K_{p, q}-L_{p, q}$. Consequently, none of these elements can be equal either to $\Im$ or to $(\Delta, 1)$. Next, developing the same arguments as in subcase IV.i, based again on Claim 4.1, we further establish that if $\mu\left(x_{\kappa}\right)=2$ for some $\kappa \in\{1,2, \ldots, t\}$, then the element ${ }^{s} \tilde{r}_{v\left(x_{\kappa}\right) \mu\left(x_{\kappa}\right)}$ must be distinct from $\left({ }^{1} \Delta^{-1}, b^{2}\right)$, if $\nu\left(x_{\kappa}\right)=s$ for some $\kappa \in$ 


$\left(\begin{array}{rrrrrrrr}\left(\Delta, b^{-1}\right) & \Im & \left(\Delta, b^{-1}\right) & \left(\Delta, b^{-1}\right) & \left(\Delta, b^{-1}\right) & \left(\Delta, b^{-1}\right) & \left(\Delta, b^{-1}\right) & \left({ }^{-1} \Delta, b^{-2}\right) \\ \left({ }^{-1} \Delta, b^{-2}\right) & \Im & \left({ }^{-1} \Delta \cdot{ }^{-2} \Delta, b^{-3}\right) & \left({ }^{-1} \Delta, b^{-2}\right) & \left({ }^{-1} \Delta, b^{-2}\right) & \left({ }^{-1} \Delta, b^{-2}\right) & \left({ }^{-1} \Delta, b^{-2}\right) & \left({ }^{-2} \Delta, b^{-3}\right) \\ \left(\Delta, b^{-1}\right) & \Im & \left({ }^{1} \Delta, 1\right) & \left(\Delta \cdot{ }^{-1} \Delta, b^{-2}\right) & \left(\Delta, b^{-1}\right) & \left(\Delta, b^{-1}\right) & \left(\Delta, b^{-1}\right) & \left({ }^{-1} \Delta, b^{-2}\right) \\ \left(\Delta, b^{-1}\right) & \Im & \left(\Delta, b^{-1}\right) & \left({ }^{1} \Delta, 1\right) & \left(\Delta \cdot{ }^{-1} \Delta, b^{-2}\right) & \left(\Delta, b^{-1}\right) & \left(\Delta, b^{-1}\right) & \left({ }^{-1} \Delta, b^{-2}\right) \\ \left(\Delta, b^{-1}\right) & \Im & \left(\Delta, b^{-1}\right) & \left(\Delta, b^{-1}\right) & \left({ }^{1} \Delta, 1\right) & \left(\Delta \cdot{ }^{-1} \Delta, b^{-2}\right) & \left(\Delta, b^{-1}\right) & \left({ }^{-1} \Delta, b^{-2}\right) \\ \left(\Delta, b^{-1}\right) & \Im & \left(\Delta, b^{-1}\right) & \left(\Delta, b^{-1}\right) & \left(\Delta, b^{-1}\right) & \left({ }^{1} \Delta, 1\right) & \left(\Delta \cdot{ }^{-1} \Delta, b^{-2}\right) & \left({ }^{-1} \Delta, b^{-2}\right) \\ \left(\Delta, b^{-1}\right) & \Im & \left(\Delta, b^{-1}\right) & \left(\Delta, b^{-1}\right) & \left(\Delta, b^{-1}\right) & \left(\Delta, b^{-1}\right) & \left({ }^{1} \Delta, 1\right) & \left(\Delta \cdot{ }^{-2} \Delta, b^{-3}\right) \\ \Im & \Im & \Im & \Im & \Im & \Im & \Im & \Im\end{array}\right)$

Figure 8. The sandwich matrix $\bar{R}_{7}^{71}$.

$\{1,2, \ldots, t\}$, then the element ${ }^{s} \tilde{r}_{v\left(x_{\kappa}\right) \mu\left(x_{\kappa}\right)}$ must be distinct from $\left({ }^{1} \Delta^{-1}, b^{2}\right)$, if $\mu\left(x_{\kappa}\right)=2$ for some $\kappa \in\{2, \ldots, t\}$, then the element ${ }^{s} \tilde{r}_{v\left(x_{\kappa-1}\right) \mu\left(x_{\kappa}\right)}$ must be distinct from $\left({ }^{1} \Delta^{-1}, b^{2}\right)$, if $v\left(x_{\kappa-1}\right)=s$ for some $\kappa \in\{2, \ldots, t\}$, then the element ${ }^{s} \tilde{r}_{v\left(x_{\kappa-1}\right) \mu\left(x_{\kappa}\right)}$ must be distinct from $\left({ }^{1} \Delta^{-1}, b^{2}\right)$, if $\mu\left(x_{1}\right)=2$ then the element ${ }^{s} \tilde{r}_{v\left(x_{t}\right) \mu\left(x_{1}\right)}$ must be distinct from $\left({ }^{1} \Delta^{-1}, b^{2}\right)$, and if $v\left(x_{t}\right)=s$ then the element ${ }^{s} \tilde{r}_{\nu\left(x_{t}\right) \mu\left(x_{1}\right)}$ must be distinct from $\left({ }^{1} \Delta^{-1}, b^{2}\right)$. Moreover, virtually the same arguments reveal that if $\mu\left(x_{\kappa}\right)=0$ or if $\mu\left(x_{\kappa}\right) \in\{3, \ldots, s\}$ for some $\kappa \in\{1,2, \ldots, t\}$, then the element ${ }^{2} \tilde{r}_{v\left(x_{\kappa}\right) \mu\left(x_{\kappa}\right)}$ must be distinct from $(\boldsymbol{I}, b)$, and also the element ${ }^{s} \tilde{r}_{v\left(x_{\kappa-1}\right) \mu\left(x_{\kappa}\right)}$ must then be distinct from $(\boldsymbol{I}, b)$ provided that $\kappa \neq 1$, and if $\mu\left(x_{1}\right)=0$ or if $\mu\left(x_{1}\right) \in\{3, \ldots, s\}$, then the element ${ }^{s} \tilde{r}_{\nu\left(x_{t}\right) \mu\left(x_{1}\right)}$ must be distinct from $(\mathbb{I}, b)$. Having this clarified, we further see that the same argument as the one used in subcase IV.ii shows that if $\mu\left(x_{\kappa}\right) \in\{3, \ldots, s-1\}$ for some $\kappa \in\{1,2, \ldots, t\}$, then the element ${ }^{s} \tilde{r}_{\nu\left(x_{\kappa}\right) \mu\left(x_{\kappa}\right)}$ must be distinct from $\left(\mathbb{I}, b^{2}\right)$, and also the element ${ }^{s} \tilde{r}_{\nu\left(x_{\kappa-1}\right) \mu\left(x_{\kappa}\right)}$ must then be distinct from $\left(\mathbb{I}, b^{2}\right)$ provided that $\kappa \neq 1$, and if $\mu\left(x_{1}\right) \in\{3, \ldots, s-1\}$, then the element ${ }^{s} \tilde{r}_{v\left(x_{t}\right) \mu\left(x_{1}\right)}$ must be distinct from $\left(\mathbb{I}, b^{2}\right)$. Quite the same argument (dealing with the elements of the column of the matrix $\bar{R}_{s}^{11}$ indexed by $s$ ) reveals that if $\mu\left(x_{\kappa}\right)=s$ for some $\kappa \in\{1,2, \ldots, t\}$, then the element ${ }^{s} \tilde{r}_{\nu\left(x_{\kappa}\right) \mu\left(x_{\kappa}\right)}$ must be distinct from $\left({ }^{1} \Delta^{-1}, b^{3}\right)$, and also the element ${ }^{s} \tilde{r}_{v\left(x_{\kappa-1}\right) \mu\left(x_{\kappa}\right)}$ must then be distinct from $\left({ }^{1} \Delta^{-1}, b^{3}\right)$ provided that $\kappa \neq 1$, and if $\mu\left(x_{1}\right)=s$, then the element ${ }^{s} \tilde{r}_{v\left(x_{t}\right) \mu\left(x_{1}\right)}$ must be distinct from $\left({ }^{1} \Delta^{-1}, b^{3}\right)$. An analogous argument (dealing with the elements of the row of the matrix $\bar{R}_{s}^{11}$ indexed by 2 ) verifies that also the element $\left({ }^{2} \Delta^{-1}, b^{3}\right)$ is likewise unusable. The only element of the matrix $\bar{R}_{s}^{11}$ which has remained, namely $\left({ }^{1} \Delta^{-1} \cdot{ }^{2} \Delta^{-1}, b^{3}\right)$, is therefore equally unusable as a possible value of any of the elements $s \tilde{r}_{v\left(x_{\kappa}\right) \mu\left(x_{\kappa}\right)}$, for $\kappa \in\{1,2, \ldots, t\}$, ${ }^{s} \tilde{r}_{v\left(x_{\kappa-1}\right) \mu\left(x_{\kappa}\right)}$, for $\kappa \in\{2, \ldots, t\}$, and ${ }^{s} \tilde{r}_{v\left(x_{t}\right) \mu\left(x_{1}\right)}$. This documents the impossibility to reach the inequality $\widehat{\chi}_{11}^{\pi}\left(\omega_{t}\right) \neq \Im$, yielding thus the desired contradiction. Hence it turns out that $\widehat{\chi}_{11}^{\pi}\left(\omega_{t}\right)=\Im$ holds again, as required.

IV.v. Assume that $k=1$ and $\ell=s$.

Then $\pi(z)=(1, s)$, the sandwich matrix $\bar{R}_{s}^{s 1}$ is normalized at the pair $(s, 1)$, and ${ }^{s} r_{s 1}=$ $\left(\Delta, b^{-1}\right)$. This sandwich matrix is of the form $\bar{R}_{s}^{s 1}=\left({ }^{s} r_{i 1}^{-1}{ }^{s} r_{i j}{ }^{s} r_{s j}^{-1}{ }^{s} r_{s 1}\right)_{i, j \in J_{s}}$. For the same reasons as before, it will again be adequate to inspect the entries of the sandwich matrix $\bar{R}_{s}^{s 1}$. For $s=7$ this sandwich matrix is shown in Figure 8, whence it is fairly easy to deduce its form for general values of the prime number $s$. Despite the fact that the individual entries of the matrix $\bar{R}_{s}^{s 1}$ look different from those of the matrix $\bar{R}_{s}^{11}$ appearing in the previous subcase, the overall structure of the matrix $\bar{R}_{s}^{s 1}$ is very much like that of the matrix $\bar{R}_{s}^{11}$. Therefore this last subcase can be treated using arguments that are quite analogous to those applied in the previous subcase. In this 
manner, one checks once again that, this time, the assumption that $\widehat{\chi}_{s 1}^{\pi}\left(\omega_{t}\right) \neq \mathfrak{s}$ leads to a contradiction. Thus the equality $\widehat{\chi}_{s 1}^{\pi}\left(\omega_{t}\right)=\Im$ holds, as required.

Remember, in conclusion, that from Theorem 1.1 and Theorem 5.1 the following consequence follows immediately.

COROLlARY 5.5. For arbitrary prime numbers $p, q$ such that $p \neq q$, there exist $2 \$_{0}$ varieties of completely simple semigroups occurring in the interval between the variety $\mathcal{R} \mathcal{B}$ of all rectangular bands and the variety $\mathcal{C S}\left(\mathcal{A}_{p} \circ \mathcal{A}_{q}\right)$ of all completely simple semigroups whose maximal subgroups belong to $\mathcal{A}_{p} \circ \mathcal{A}_{q}$.

\section{REFERENCES}

1. D. E. Cohen, On the laws of a metabelian variety, J. Algebra 5 (1967), 267-273.

2. D. G. Fitz-Gerald, On inverses of products of idempotents in regular semigroups, J. Austral. Math. Soc. 13 (1972), 335-337.

3. G. Higman, Some remarks on varieties of groups, Quart. J. Math. Oxford Ser. (2) 10 (1959), 165-178.

4. Hanna Neumann, Varieties of groups (Springer-Verlag, 1967).

5. S. Oates and M. B. Powell, Identical relations in finite groups, J. Algebra 1 (1964), 11-39.

6. M. Petrich and N. R. Reilly, Near varieties of idempotent generated completely simple semigroups, Algebra Universalis 16 (1983), 83-104.

7. M. Petrich and N. R. Reilly, All varieties of central completely simple semigroups, Trans. Amer. Math. Soc. 280 (1983), 623-636.

8. M. Petrich and N.R. Reilly, Completely regular semigroups (John Wiley \& Sons, New York, 1999). $1-30$.

9. L. Polák, On varieties of completely regular semigroups III, Semigroup Forum 37 (1988), 